University of Tennessee Health Science Center

UTHSC Digital Commons

\title{
A Pharmacokinetics and Pharmacodynamics (PK/PD) Guided Approach to Lead Optimization of Nitrofuranylamide Anti- Tuberculosis Agents
}

Nageshwar Rao Budha

University of Tennessee Health Science Center

Follow this and additional works at: https://dc.uthsc.edu/dissertations

Part of the Medicinal and Pharmaceutical Chemistry Commons, and the Pharmaceutics and Drug Design Commons

\section{Recommended Citation}

Budha, Nageshwar Rao, "A Pharmacokinetics and Pharmacodynamics (PK/PD) Guided Approach to Lead Optimization of Nitrofuranylamide Anti-Tuberculosis Agents" (2009). Theses and Dissertations (ETD). Paper 329. http://dx.doi.org/10.21007/etd.cghs.2009.0039. 


\title{
A Pharmacokinetics and Pharmacodynamics (PK/PD) Guided Approach to Lead Optimization of Nitrofuranylamide Anti-Tuberculosis Agents
}

\begin{abstract}
Currently used treatment strategies for tuberculosis (TB) involve administration of multiple drug combinations for a minimum of 6-9 months. However, these prolonged regimens do not always achieve sterilization, as evidenced by post-therapy relapse in a subgroup of treated individuals. In an effort to develop novel therapeutic agents for TB a new class of chemical agents, nitrofuranylamides, is being developed at the University of Tennessee Health Science Center. We hypothesized that the application of an iterative pharmacokinetics and pharmacodynamics (PK/PD) guided approach would facilitate the optimization of nitrofuranylamide lead compounds suitable for further development.
\end{abstract}

First, we examined the biopharmaceutic properties and preclinical PK of nitrofuranylamide lead compounds. The first tested lead compound, Lee 562, exhibited a high systemic clearance, short terminal half-life, and low oral bioavailability of $15.9 \%$. These observations were further supported by the poor metabolic stability of Lee 562. Thus, two second generation follow-up compounds, Lee 878 and Lee 952 were tested that included structural modifications for increased metabolic stability. Both compounds showed improved metabolic stability compared to Lee 562. As expected, this in vitro observation translated into an increased in vivo stability (lower plasma clearance) of Lee 878 compared to Lee 562 and Lee 952 , with a 20 - and 10 -fold higher systemic exposure, respectively. As a consequence, oral bioavailability of Lee 878 reached $\sim 27 \%$ compared to $16 \%$ for Lee 952 and Lee 562 . We then tested a new set of nine third generation compounds for microsomal metabolic stability to guide selection of a stable compound with even higher oral bioavailability. From this panel Lee 1106 was selected for further PK evaluation in rats. Lee 1106 exhibited favorable PK properties such as a low systemic clearance and a long terminal half-life. However, oral bioavailability of Lee 1106 was poor (4.6\%). Biopharmaceutic evaluation of the compound showed that the compound has poor aqueous solubility and a high clogP. Further, the plasma protein binding of the compound was found to be high underlining the hydrophobicity of the molecule. Based on these results, a PK guided screening paradigm was developed for optimization of the nitrofuranylamide lead compounds.

In order to add pharmacodynamic considerations to the lead optimization approach, we developed a novel in vitro PK/PD model and validated the model by studying the activity of a first line anti-TB drug, isoniazid (INH) against M. bovis BCG as a model organism. M. bovis BCG in the model was treated with multiple doses of INH mimicking concentration-time profiles encountered during multiple dosing in vivo. The time-kill data was analyzed using a semi-mechanistic PK/PD model that included an adaptive IC50 function for explaining the re-growth of bacteria observed over the course of treatment. The PK/PD model was able to describe the data well. The PK/PD index $A_{U} \mathrm{C}_{0-24} / \mathrm{MIC}$ was found to be the most explanatory of antimicrobial effect of INH. These findings are in agreement with the previously published studies and suggest that the in vitro PK/PD model can be used for assessing the antimycobacterial activity of lead compounds.

Thus, the in vitro PK/PD model was subsequently applied to establish time-kill curves for dosing regimens of lead compound Lee 1106. Lee 1106 showed exposure-dependent killing of M. bovis BCG. A semimechanistic PK/PD model was developed to describe the Lee 1106 mediated killing of bacteria. The model was subsequently used for numerical simulation experiments to predict the killing effect of different untested multiple dose regimens of Lee 1106 in mice. It was found that the once daily regimen is most adequate for further studies in the mouse model of TB. The information gained from the PK/PD evaluation and the simulation experiments illustrate the utility of the PK/PD guided approach for the selection of compounds with favorable properties for a high likelihood of in vivo efficacy. 
In summary, we have successfully developed an iterative PK/PD guided process for lead optimization of nitrofuranylamides that uses a set of biopharmaceutic, pharmacokinetic and pharmacodynamic evaluations as criteria for compound and dose selection to move to subsequent development levels.

\section{Document Type}

Dissertation

Degree Name

Doctor of Philosophy (PhD)

\section{Program}

Pharmaceutical Sciences

\section{Research Advisor}

Bernd Meibohm, Ph.D.

\section{Keywords}

Tuberculosis, Pharmacokinetics, Pharmacodynamics, Drug development, In vitro PK/PD model and Timekill analysis

\section{Subject Categories}

Medicinal and Pharmaceutical Chemistry | Medicine and Health Sciences | Pharmaceutics and Drug Design | Pharmacy and Pharmaceutical Sciences 


\title{
A PHARMACOKINETICS AND PHARMACODYNAMICS (PK/PD) GUIDED APPROACH TO LEAD OPTIMIZATION OF NITROFURANYLAMIDE ANTI-TUBERCULOSIS AGENTS
}

\author{
A Dissertation \\ Presented for \\ The Graduate Studies Council \\ The University of Tennessee \\ Health Science Center
}

\author{
In Partial Fulfillment \\ Of the Requirements for the Degree \\ Doctor of Philosophy \\ From The University of Tennessee
}

By

Nageshwar Rao Budha

May 2009 
Chapter 1 (C) 2008 by Bentham Science Publishers.

Chapter 2 (C) 2008 by Springer.

All other material (c) 2009 by Nageshwar R. Budha. 
For my parents for their endless love and continuous support

$$
\text { and }
$$

For my wife for her love and support 


\section{ACKNOWLEDGEMENTS}

I could never have completed this daunting task without the help, support, guidance and efforts of a lot of people. First and foremost, I would like to express my deepest gratitude and sincere appreciation to my mentor Dr. Bernd Meibohm for providing me with an opportunity to work in his lab and instilling in me the qualities of being a good scientist. His co-operation and continued support have been major driving forces through my graduate career at the University of Tennessee. I would also like to thank my committee members, Dr. Richard Lee, Dr. Charles Ryan Yates, Dr. Phillip D. Rogers and Dr. James E. Bina for their invaluable suggestions, guidance and assistance over the years. I would like to especially acknowledge Dr. Richard Lee for providing me with the compounds and allowing me to use his laboratory without which my dissertation work would not have been possible.

I would like to thank Robin and Julian for their support and helpful suggestions during the microbiology experiments. A special thank you to the members of Dr. Richard Lee's group at the University of Tennessee for their support, help and encouragement. Thanks to Michael Nguyen, Biomedical Instrumentation facility at the University of Tennessee for making custom tools in the design of the in vitro PK/PD model.

A special thank you to my friends and colleagues, Nate, Yi, Maggie, Fei, Les, Satyendra, Pavan, Karin, Wararat, and Josiah for their support and wonderful company over all these years. I would also like to thank my colleagues who graduated before me and my previous lab members including, Manish, Lisa, Raghu, Pengfei, Nitin, and Shen for their guidance and support. I also extend my gratitude to my friends for being friends and for making my stay in Memphis a memorable experience.

I would like to thank the University of Tennessee Health Science Center for providing me with the opportunity to pursue graduate education.

I would like to thank my parents and my wife; no words would be enough to thank their support and patience during the completion of this dissertation. I would like to thank all my family members for their love and support. I would like to thank all my teachers from the kindergarten to the graduate school for igniting in me the light of knowledge. Finally, I would like to thank Almighty for everything that I have today. 


\begin{abstract}
Currently used treatment strategies for tuberculosis (TB) involve administration of multiple drug combinations for a minimum of 6-9 months. However, these prolonged regimens do not always achieve sterilization, as evidenced by post-therapy relapse in a subgroup of treated individuals. In an effort to develop novel therapeutic agents for TB a new class of chemical agents, nitrofuranylamides, is being developed at the University of Tennessee Health Science Center. We hypothesized that the application of an iterative pharmacokinetics and pharmacodynamics (PK/PD) guided approach would facilitate the optimization of nitrofuranylamide lead compounds suitable for further development.

First, we examined the biopharmaceutic properties and preclinical PK of nitrofuranylamide lead compounds. The first tested lead compound, Lee 562, exhibited a high systemic clearance, short terminal half-life, and low oral bioavailability of $15.9 \%$. These observations were further supported by the poor metabolic stability of Lee 562 . Thus, two second generation follow-up compounds, Lee 878 and Lee 952 were tested that included structural modifications for increased metabolic stability. Both compounds showed improved metabolic stability compared to Lee 562. As expected, this in vitro observation translated into an increased in vivo stability (lower plasma clearance) of Lee 878 compared to Lee 562 and Lee 952, with a 20- and 10-fold higher systemic exposure, respectively. As a consequence, oral bioavailability of Lee 878 reached $\sim 27 \%$ compared to $16 \%$ for Lee 952 and Lee 562 . We then tested a new set of nine third generation compounds for microsomal metabolic stability to guide selection of a stable compound with even higher oral bioavailability. From this panel Lee 1106 was selected for further PK evaluation in rats. Lee 1106 exhibited favorable PK properties such as a low systemic clearance and a long terminal half-life. However, oral bioavailability of Lee 1106 was poor $(4.6 \%)$. Biopharmaceutic evaluation of the compound showed that the compound has poor aqueous solubility and a high clogP. Further, the plasma protein binding of the compound was found to be high underlining the hydrophobicity of the molecule. Based on these results, a PK guided screening paradigm was developed for optimization of the nitrofuranylamide lead compounds.

In order to add pharmacodynamic considerations to the lead optimization approach, we developed a novel in vitro $\mathrm{PK} / \mathrm{PD}$ model and validated the model by studying the activity of a first line anti-TB drug, isoniazid (INH) against M. bovis BCG as a model organism. M. bovis BCG in the model was treated with multiple doses of INH mimicking concentration-time profiles encountered during multiple dosing in vivo. The time-kill data was analyzed using a semi-mechanistic PK/PD model that included an adaptive IC50 function for explaining the re-growth of bacteria observed over the course of treatment. The PK/PD model was able to describe the data well. The PK/PD index $\mathrm{AUC}_{0-24} / \mathrm{MIC}$ was found to be the most explanatory of antimicrobial effect of INH. These findings are in agreement with the previously published studies and suggest that the in vitro $\mathrm{PK} / \mathrm{PD}$ model can be used for assessing the antimycobacterial activity of lead compounds.
\end{abstract}


Thus, the in vitro PK/PD model was subsequently applied to establish time-kill curves for dosing regimens of lead compound Lee 1106. Lee 1106 showed exposuredependent killing of $M$. bovis BCG. A semi-mechanistic PK/PD model was developed to describe the Lee 1106 mediated killing of bacteria. The model was subsequently used for numerical simulation experiments to predict the killing effect of different untested multiple dose regimens of Lee 1106 in mice. It was found that the once daily regimen is most adequate for further studies in the mouse model of TB. The information gained from the $\mathrm{PK} / \mathrm{PD}$ evaluation and the simulation experiments illustrate the utility of the PK/PD guided approach for the selection of compounds with favorable properties for a high likelihood of in vivo efficacy.

In summary, we have successfully developed an iterative $\mathrm{PK} / \mathrm{PD}$ guided process for lead optimization of nitrofuranylamides that uses a set of biopharmaceutic, pharmacokinetic and pharmacodynamic evaluations as criteria for compound and dose selection to move to subsequent development levels. 


\section{TABLE OF CONTENTS}

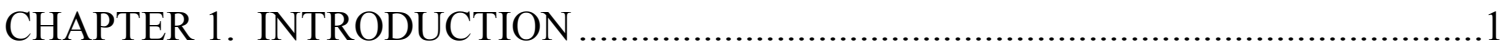

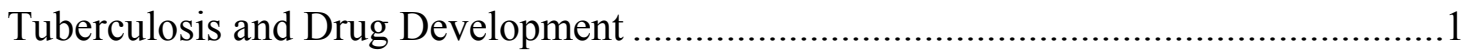

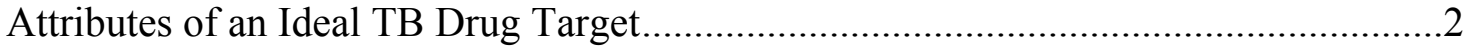

Different Targets Available for TB Therapy: Old and New ........................................

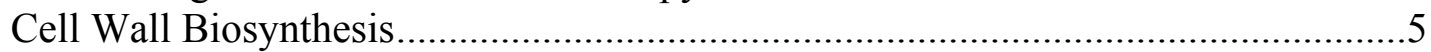

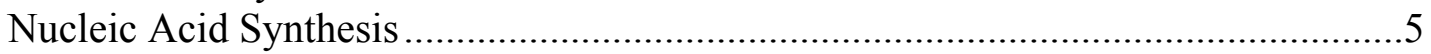

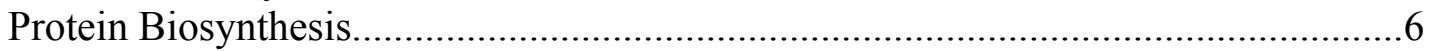

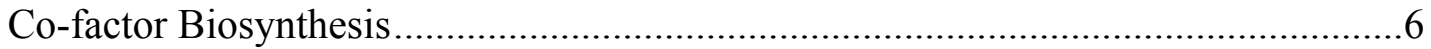

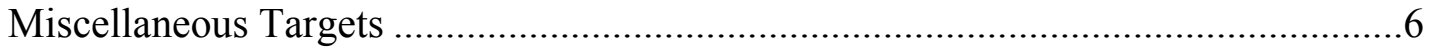

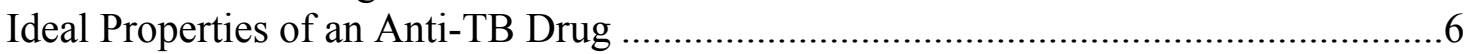

Biopharmaceutic Properties ..................................................................................

Pharmacokinetic Properties .............................................................................

Pharmacodynamic Properties................................................................................

Biopharmaceutic, Pharmacokinetic, and Pharmacodynamic Properties of Anti-TB

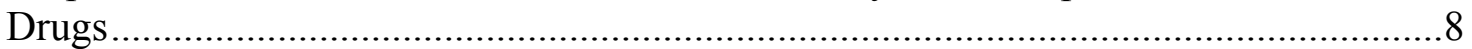

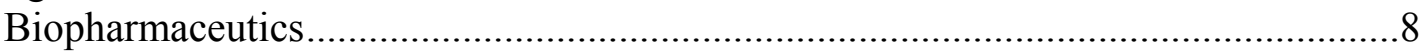

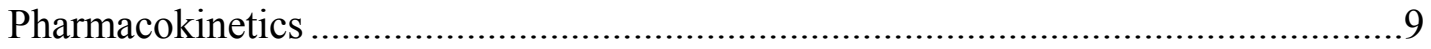

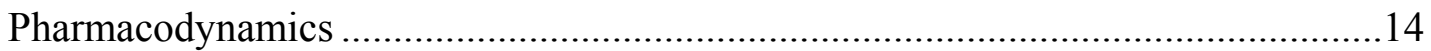

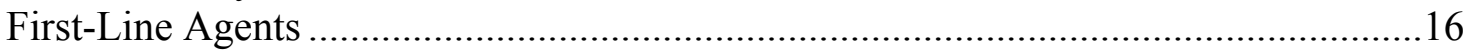

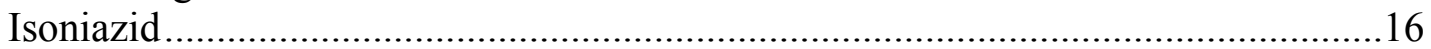

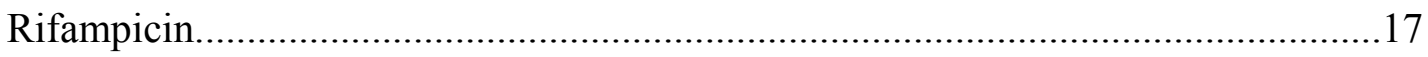

Pyrazinamide.....................................................................................19

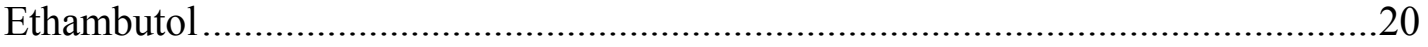

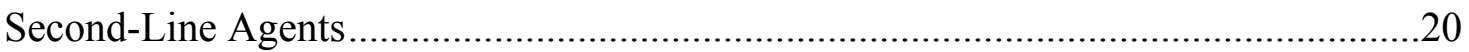

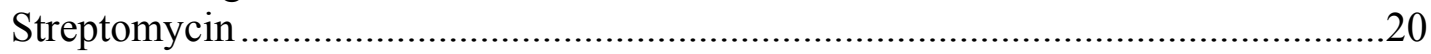

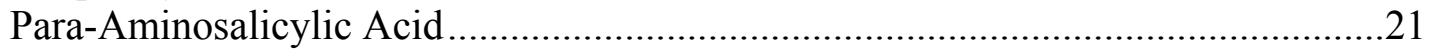

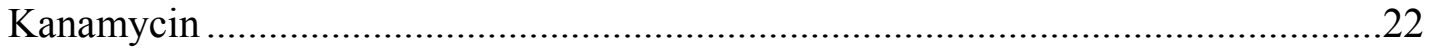

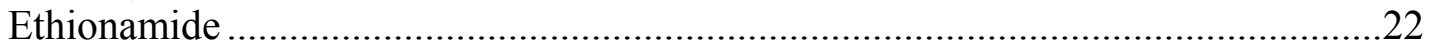

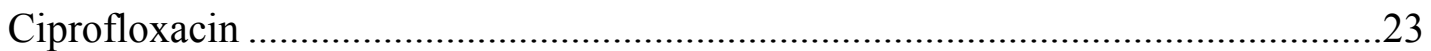

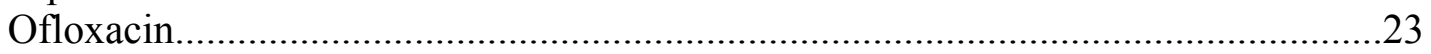

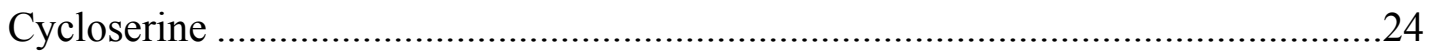

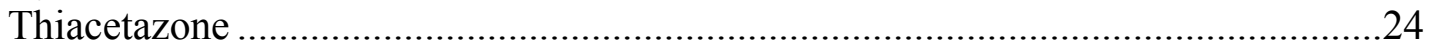

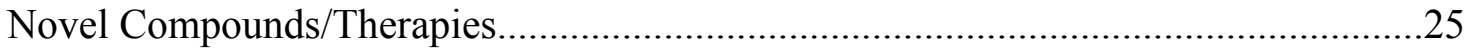

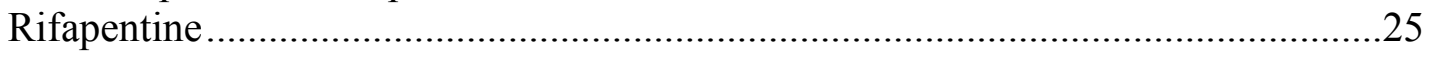

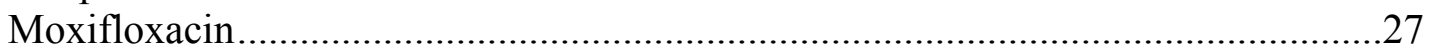

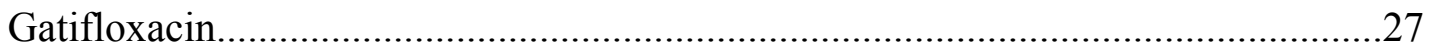

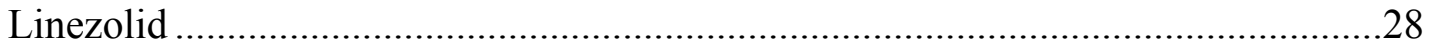

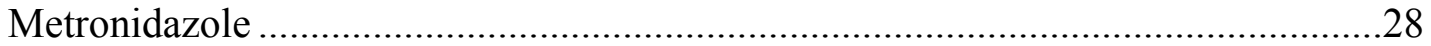

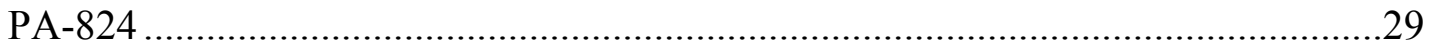

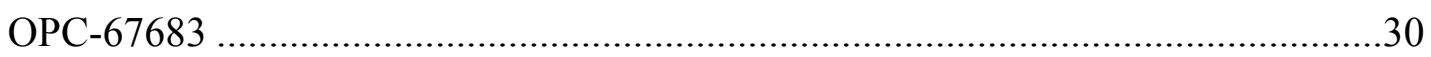

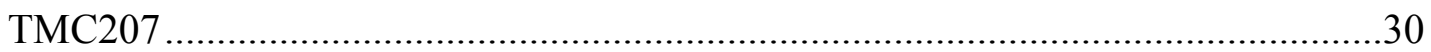

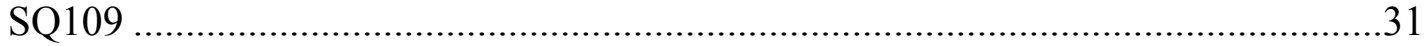

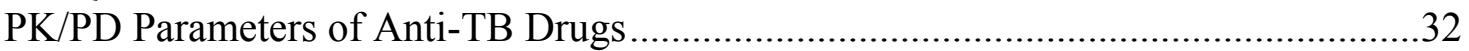


Central Hypothesis .............................................................................................

\section{CHAPTER 2. PHARMACOKINETICALLY-GUIDED LEAD OPTIMIZATION OF} NITROFURANYLAMIDE ANTI-TUBERCULOSIS AGENTS .............................38

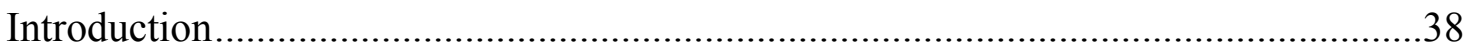

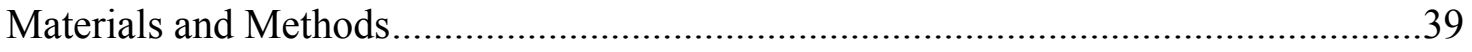

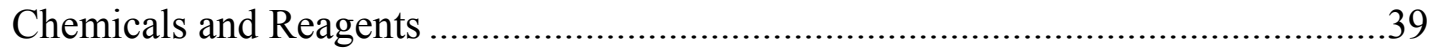

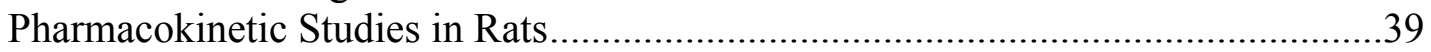

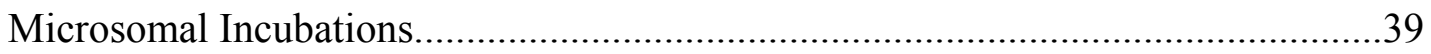

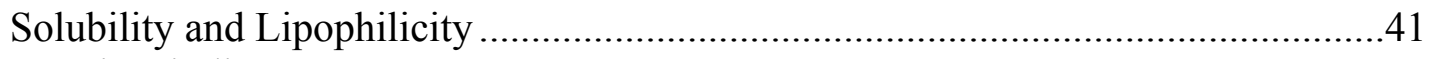

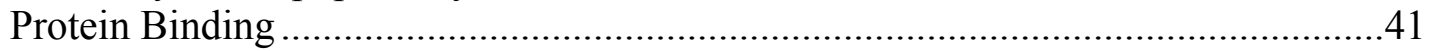

Plasma Concentration Measurements by LC-MS/MS .........................................41

Pharmacokinetic Data Analysis ........................................................................... 42

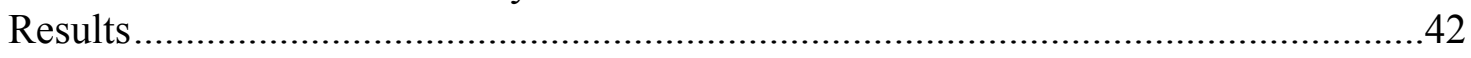

Pharmacokinetics of Lee 562 ......................................................................42

Microsomal Metabolic Stability of Second Generation Compounds Lee 878 and

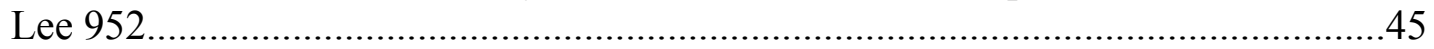

Pharmacokinetics of Second Generation Compounds Lee 878 and Lee 952 ...........45

Metabolic Stability of Third Generation Compounds .........................................48

Pharmacokinetics of the Third Generation Compound Lee 1106 ...........................48

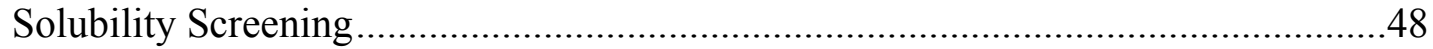

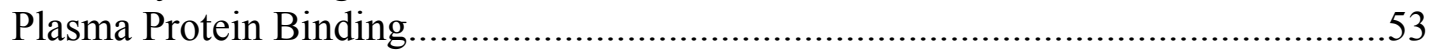

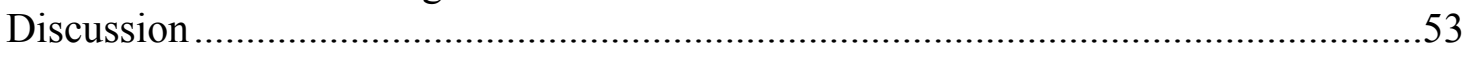

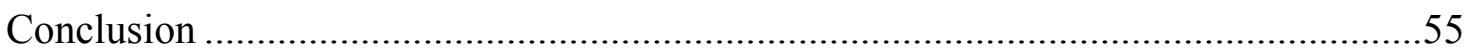

CHAPTER 3. AN IN VITRO PK/PD MODEL TO DETERMINE TIME-KILL CURVES OF ANTIBIOTICS AGAINST SLOW-GROWING

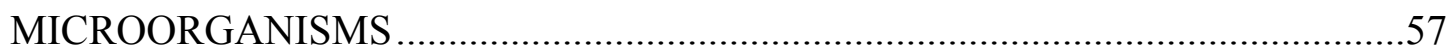

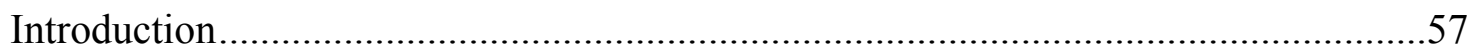

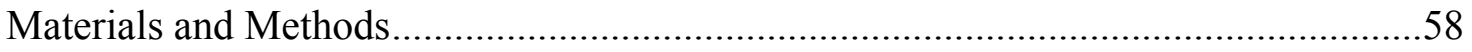

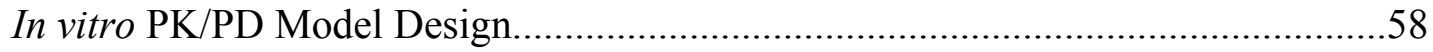

Culture, Media, and Antibiotics ........................................................................60

Minimum Inhibitory Concentration of Isoniazid ................................................6

Time-Kill Curves of INH................................................................................61

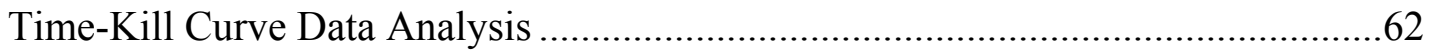

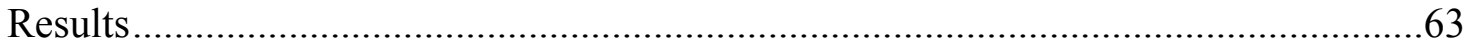

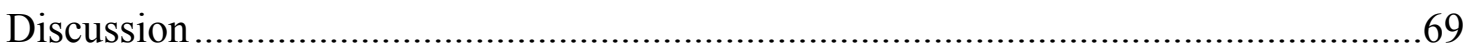

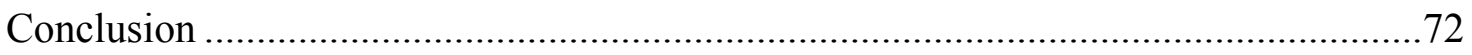

\section{CHAPTER 4. PHARMACODYNAMICS OF LEE 1106 AGAINST M. BOVIS BCG}

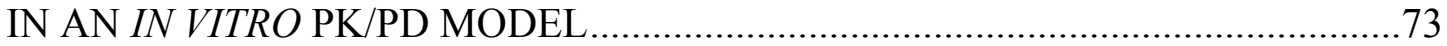

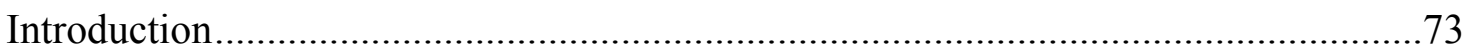

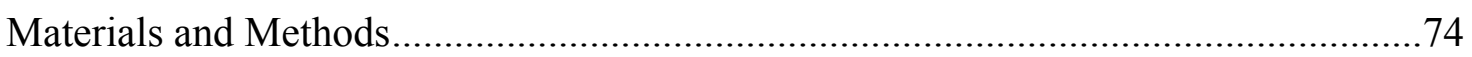

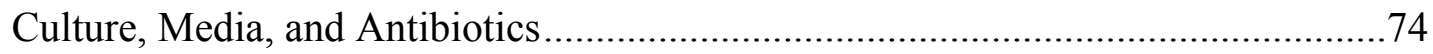

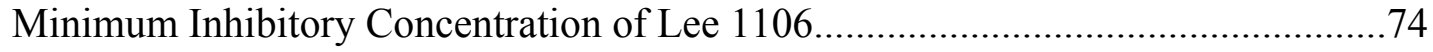

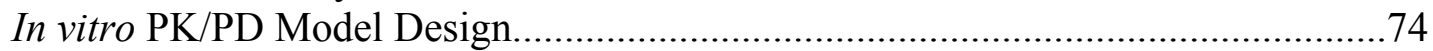


Time-Kill Curves of Lee 1106

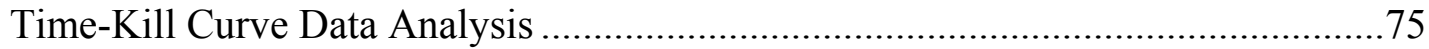

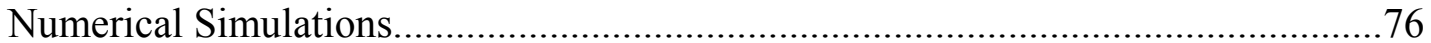

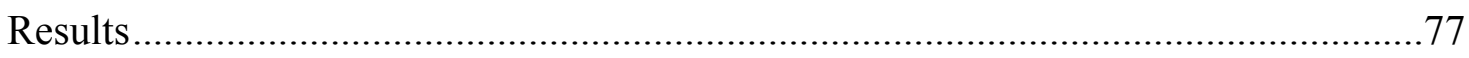

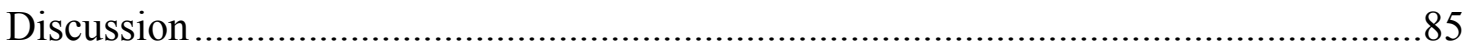

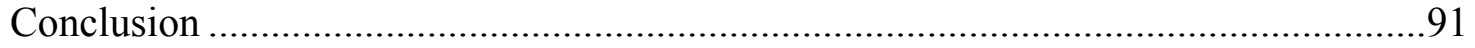

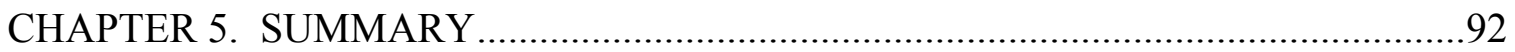

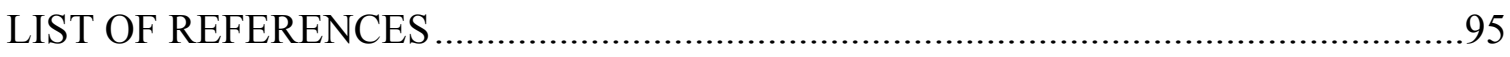

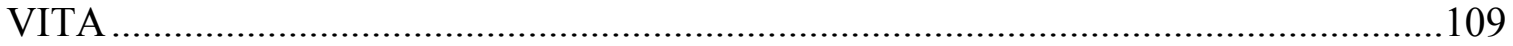




\section{LIST OF TABLES}

Table 1-1. Existing Anti-Tuberculosis Drug Targets...............................................

Table 1-2. Biopharmaceutic Properties of Anti-Tuberculosis Drugs.........................10

Table 1-3. Pharmacokinetic Parameters of Anti-Tuberculosis Drugs........................11

Table 1-4. Biopharmaceutic and Pharmacokinetic Properties of Investigational Anti-Tuberculosis Drugs........................................................................33

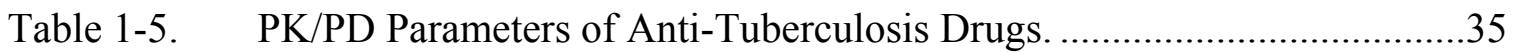

Table 2-1. Pharmacokinetic Parameters (Mean \pm SD) of Lee 562, Lee 878, Lee 952, and Lee 1106.............................................................................44

Table 2-2. Aqueous Solubility and Plasma Protein Binding of Lee 562, Lee 878,

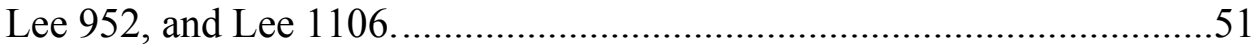

Table 3-1. Experimental Plan with Initial Concentrations of Isoniazid....................62

Table 3-2. Parameter Estimates of Isoniazid PK/PD Model...................................65

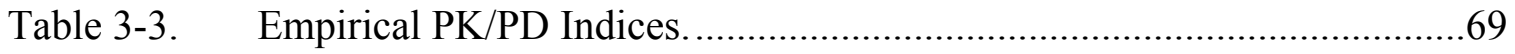

Table 4-1. Experimental Plan with Initial Concentrations of Lee 1106.....................75

Table 4-2. Parameter Estimates of Lee 1106 PK/PD Model....................................80

Table 4-3. $\quad$ Empirical PK/PD Indices Based on Day 3 Data...................................85 


\section{LIST OF FIGURES}

Figure 1-1. Chemical Structures of Established Anti-Tuberculosis Drugs...................18

Figure 1-2. Chemical Structures of Newer and Investigational Anti-Tuberculosis

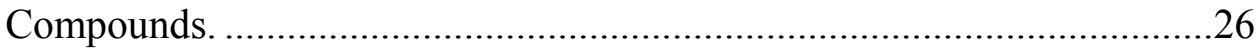

Figure 2-1. Chemical Structures of Lee 562, Lee 878, Lee 952, and Lee 1106...........40

Figure 2-2. Measured Plasma Concentration-Time Profiles (Mean \pm SD) after Intravenous (10 mg/kg, Panel A) or Oral (100 mg/kg, Panel B) Administration of Lee 562 to Rats

Figure 2-3. Measured Plasma Concentration-Time Profiles (Mean \pm SD) after Intravenous (10 mg/kg, Panel A) or Oral (100 mg/kg, Panel B) Administration of Lee 878 to Rats

Figure 2-4. Measured Plasma Concentration-Time Profiles (Mean \pm SD) after Intravenous (10 mg/kg, Panel A) or Oral (100 mg/kg, Panel B) Administration of Lee 952 to Rats

Figure 2-5. Chemical Structures of Third Generation Nitrofuranylamides

Figure 2-6. Measured Plasma Concentration-Time Profiles (Mean \pm SD) after Intravenous (10 mg/kg, Panel A) or Oral (100 mg/kg, Panel B) Administration of Lee 1106 to Rats

Figure 2-7. Relationship between Aqueous Solubility, Plasma Protein Binding and clogP in the Investigated Nitrofuranylamides.

Figure 2-8. Screening Paradigm for Lead Optimization of Anti-TB Compounds. .......56

Figure 3-1. Schematic Diagram of In vitro PK/PD Model..........................................59

Figure 3-2. A Picture Showing Two Working Units of In vitro PK/PD Model...........61

Figure 3-3. Plots Showing Measured (Mean \pm SD) and Model Predicted (Lines) M. bovis BCG CFU/mL over Time for the PK/PD Model.

Figure 3-4. Goodness-of-Fit Plots for the INH PK/PD Model - Observed versus Predicted Values.

Figure 3-5. Goodness-of-Fit Plots for the INH PK/PD Model - Weighted Residuals. 
Figure 3-6. Relationship between PK/PD Indices and Microbial Kill on Day 3 after Treatment.

Figure 4-1. Measured (Symbols) and PK/PD Model Predicted (Lines) M. bovis BCG CFU/mL over Time for Different Dosing Regimens of Lee 1106...78

Figure 4-2. Initial Kill Rates of Lee 1106 Plotted against Dose. 79

Figure 4-3. Goodness-of-Fit Plots for the Lee 1106 PK/PD Model - Observed versus Predicted Values.

Figure 4-4. Goodness-of-Fit Plots for the Lee 1106 PK/PD Model - Weighted Residuals.

Figure 4-5. Visual Predictive Check for the PK/PD Model.

Figure 4-6. Relationship between PK/PD Indices and Microbial Kill on Day 3 after Treatment.

Figure 4-7. 90\% Prediction Intervals (Dotted Lines) for Time-Kill Curves Calculated from 1000 Numerical Simulations for Once Daily Dosing Regimens of Lee 1106 in Mice.

Figure 4-8. 90\% Prediction Intervals (Dotted Lines) for Time-Kill Curves Calculated from 1000 Numerical Simulations for Twice Daily Dosing Regimens of Lee 1106 in Mice.

Figure 4-9. $\quad 90 \%$ Prediction Intervals (Dotted Lines) for Time-Kill Curves Calculated from 1000 Numerical Simulations for Once Weekly Dosing Regimens of Lee 1106 in Mice.

Figure 4-10. 90\% Prediction Intervals (Dotted Lines) for Time-Kill Curves Calculated from 1000 Numerical Simulations for Twice Weekly Dosing Regimens of Lee 1106 in Mice. 


\section{LIST OF ABBREVIATIONS}

BCS

CFU

CIP

CNS

$\mathrm{CS}$

CSF

CYP

EBA

EMB

ETH

FASII

FDA

FQ

HIV

HTS

ICL

INH

KM

LTB

MAC

MBC

MDR-TB

MIC

M.tb

MW

NIAID

$\mathrm{NIH}$

OFL

PAS

PD

PK

POA

PZA

RIF

SM

TB

TBTC

WHO

XDR-TB
Biopharmaceutics Classification System

Colony Forming Units

Ciprofloxacin

Central Nervous System

Cycloserine

Cerebrospinal Fluid

Cytochrome P450

Early Bactericidal Activity

Ethambutol

Ethionamide

Fatty Acid Synthase II

Food and Drug Administration

Fluoroquinolones

Human Immunodeficiency Virus

High Throughput Screening Isocitrate Lyase Isoniazid

Kanamycin

Latent Tuberculosis

Mycobacterium avium Complex

Minimum Bactericidal Concentration

Multi Drug Resistant Tuberculosis

Minimum Inhibitory Concentration

Mycobacterium tuberculosis

Molecular Weight

National Institute of Allergy and Infectious Diseases

National Institutes of Health

Ofloxacin

Para-Aminosalicylic Acid

Pharmacodynamics

Pharmacokinetics

Pyrazinoic Acid

Pyrazinamide

Rifampicin

Streptomycin

Tuberculosis

Tuberculosis Trials Consortium

World Health Organization

Extensively Drug Resistant Tuberculosis 


\section{CHAPTER 1. INTRODUCTION*}

\section{Tuberculosis and Drug Development}

Tuberculosis (TB) is a chronic, air-borne infectious disease caused by Mycobacterium tuberculosis (M.tb). According to the World Health Organization (WHO), one-third of the world population is currently infected with TB, and approximately $10 \%$ of these individuals are expected to develop active TB at some point in their lifetime. TB emerged as a leading killer among HIV-infected people with declined immune function. A quarter of a million TB deaths are HIV associated. The majority of TB deaths occurs in the developing world (countries of Latin America, Asia, and Africa), affecting mostly young adults in their most productive years which creates an adverse impact on global economics [1].

Currently used treatment strategies involve administration of multiple drugs for a minimum of 6 to 9 months. However, this prolonged regimen does not always achieve sterilization, as evidenced by post-therapy relapse in a subgroup of treated individuals [2]. Development of resistance to treatment is a hallmark of TB. The prevalence of multidrug resistant tuberculosis (MDR-TB) is very high in underdeveloped nations due to lack of adherence to therapy. Treatment of MDR-TB is 100 times costlier than drug sensitive $\mathrm{TB}$, increases the duration of therapy and necessitates the use of more toxic second-line drugs [1]. MDR-TB is present in all 109 countries recently surveyed by the WHO and the incidence rate of MDR-TB and XDR-TB (Extensively Drug Resistant TB) is increasing worldwide. MDR-TB is defined as TB resistant to the main first-line drugs, isoniazid and rifampicin. XDR-TB is defined as TB with MDR resistance as well as resistance to any one of the fluoroquinolone drugs and to at least one of the three injectable second-line drugs, amikacin, capreomycin or kanamycin. Although the annual incidence rate of TB cases in the United States and other industrialized nations is decreasing steadily, the increase in incidence rate of MDR and XDR-TB cases is raising concerns regarding progress towards the goal of TB eradication in these nations. The rise of MDR and XDRTB cases worldwide is also increasing concerns that we may lose control of the spread of TB $[1,3]$.

Discovery and development of antibiotics in general is in a declining phase due to the lack of commercial incentive and the perceived small market potential. This is because newly developed antibiotics are often reserved for treating only drug resistant bacterial infections which limits their market size [4]. Higher distribution of TB cases in the poorest nations of the world and steady decline in the number of TB cases in the developed world are further worsening the condition of TB drug discovery research [5]. Consequently, there is an urgent need to develop novel, fast acting anti-TB drugs with high potency that can provide treatment options for MDR and XDR-TB, reduce the

*This chapter adapted with permission. Budha, N.R., R.E. Lee, and B. Meibohm, Biopharmaceutics, pharmacokinetics and pharmacodynamics of antituberculosis drugs. Curr Med Chem, 2008. 15(8): p. 809-25 [152]. 
duration of therapy to improve adherence, and decrease the interaction potential with anti-HIV agents.

Drug discovery research has undergone remarkable changes in the last decade. Many new technologies and strategies have been developed and these developments resulted in new opportunities for accumulating detailed information about drug candidates. Examples include high throughput screening (HTS), combinatorial chemistry/ high throughput synthesis of libraries, genomics, proteomics and computational approaches [6]. HTS campaigns have produced large numbers of hits that have been subsequently optimized into high affinity ligands for many therapeutic targets. However, often this has resulted in high molecular weight compounds with poor biopharmaceutic and pharmacokinetic properties creating a major bottleneck in drug discovery research. Poor pharmacokinetic properties secondary to poor biopharmaceutical and/or physicochemical properties are accountable for the majority of failures in early drug development [7]. The in vivo activity of anti-infectives is dictated by their intrinsic antimicrobial activity and their free, unbound concentration in the target tissue, as only free, non-protein bound drug is pharmacologically active. Biopharmaceutic and pharmacokinetic properties consequently play a fundamental role in the efficacy of antibiotics, as they determine a drug's rate and extent of absorption as well as its disposition and tissue distribution profile. It has become imperative to consider biopharmaceutic properties like solubility, permeability and other physicochemical properties for increasing the success rate in developing new molecules [8,9]. This approach is now an integral part of most drug development programs [10-12].

In the following sections, we discuss the properties of a desirable TB target, the targets of current TB drugs, focus on important biopharmaceutic, pharmacokinetic, and pharmacodynamic properties desirable in antitubercular drug development, and provide a brief account on the properties of antimycobacterial medications in use and under development.

\section{Attributes of an Ideal TB Drug Target}

It is quite likely that there exists no single ideal target for tuberculosis therapy, rather tuberculosis should be viewed as an atypical infection with multiple subpopulations of bacteria showing differential drug sensitivity that may need to be targeted by a combination of different drugs. Currently it is believed that the most important subpopulations to be targeted by new anti-tuberculosis agents are actively growing and persister populations (bacterial cells that neither grow nor die in the presence of antibiotics). Thus, activity against all the subpopulations is a prerequisite for successful eradication. In order for a TB target to be 'druggable' it should be essential for the bacterium's survival, have a fully elucidated function, be amenable to a high throughput functional assay, and have no close human ortholog such that specific inhibitors can be generated [13]. The properties of current TB drug targets in relation to the outlined attributes for an ideal target are provided in Table 1-1. Desirable properties

of a TB drug target are described in the following [14]. 
Table 1-1. Existing Anti-Tuberculosis Drug Targets.

\begin{tabular}{|c|c|c|c|c|c|c|c|c|c|c|}
\hline Drug & Target & Essentiality & $\begin{array}{l}\text { Bactericidal } \\
\text { Activity }\end{array}$ & $\begin{array}{l}\text { Sterilizing } \\
\text { Activity }\end{array}$ & $\begin{array}{l}\text { Uniqueness/ } \\
\text { Selectivity }\end{array}$ & $\begin{array}{l}\text { Spectrum of } \\
\text { Activity }\end{array}$ & Testability & $\begin{array}{c}\text { 3D } \\
\text { Structure }\end{array}$ & Validity & References \\
\hline Isoniazid & $\begin{array}{l}\text { Multiple targets including } \\
\text { acyl carrier protein } \\
\text { reductase }(\operatorname{Inh} A)\end{array}$ & +++ & +++ & + & +++ & Narrow & None & Available & +++ & {$[18,19]$} \\
\hline Rifampicin & $\begin{array}{l}\text { RNA polymerase } \beta \\
\text { subunit }\end{array}$ & +++ & +++ & +++ & +++ & Medium & None & Available & +++ & [20] \\
\hline Pyrazinamide & $\begin{array}{l}\text { Membrane energy } \\
\text { metabolism }\end{array}$ & +++ & + & +++ & +++ & Narrow & None & $\begin{array}{c}\text { Not } \\
\text { available }\end{array}$ & +++ & [21] \\
\hline Ethambutol & Arabinosyl transferase & +++ & ++ & + & +++ & Narrow & None & $\begin{array}{c}\text { Not } \\
\text { available }\end{array}$ & +++ & [22] \\
\hline Streptomycin & $\begin{array}{l}\text { Ribosomal S12 protein } \\
\text { and 16S rRNA }\end{array}$ & +++ & + & + & +++ & Broad & None & Available & +++ & [23] \\
\hline Kanamycin & 16SrRNA & +++ & ++ & ++ & +++ & Broad & None & Available & +++ & {$[24,25]$} \\
\hline Moxifloxacin & DNA gyrase & +++ & +++ & ++ & ++ & Broad & None & Available & +++ & {$[26]$} \\
\hline Ethionamide & $\begin{array}{l}\text { Acyl carrier protein } \\
\text { reductase }(\operatorname{Inh} A)\end{array}$ & +++ & ++ & + & +++ & Narrow & None & Available & +++ & {$[18,19]$} \\
\hline PAS & Thymidylate synthase & +++ & + & + & ++ & Medium & $\begin{array}{l}\text { Currently } \\
\text { unknown }\end{array}$ & Available & +++ & {$[27,28]$} \\
\hline Cycloserine & D-alanine racemase & +++ & + & + & ++ & Medium & $\begin{array}{l}\text { Currently } \\
\text { unknown }\end{array}$ & Available & +++ & [29] \\
\hline Thiacetazone & Currently unknown & +++ & + & + & +++ & Narrow & None & $\begin{array}{l}\text { Currently } \\
\text { unknown }\end{array}$ & +++ & {$[30,31]$} \\
\hline
\end{tabular}


- Essentiality - desired target should be essential for bacterial growth (in vitro as well as in vivo), metabolism and viability. Inactivation of target should result in immediate bacterial death or inability to persist. Target enzyme/protein encoding gene should belong to a highly conserved region in the bacterial genome.

- Bactericidal activity - target inhibition should result in rapid killing of actively growing bacteria.

- Sterilizing activity - target inhibition should result in killing of dormant/persistent bacterial population and reduce the duration of therapy.

- Uniqueness/selectivity - desired target should not contain a direct mammalian ortholog for target specificity and avoiding host toxicity.

- Narrow spectrum of activity - corresponding target should not be available in other bacterial species to avoid emergence of drug resistance to TB because of inadvertent use of antibacterial in other infections and to avoid GI side effects from overgrowth of resistant gut flora during prolonged therapy.

- Testability - the effect of inhibiting a target should be easily testable i.e., a test system should be available for screening purposes. The target should be amenable to whole-cell or cell free high throughput screening format.

- Availability of $3 D$ structure - X-ray crystal or NMR structural information should ideally be available to guide optimization of the emerging leads.

- Validity - target should have extensive validation such that the active site is 'druggable' i.e., that suitably sized small molecule inhibitors with drug like properties can bind to it efficiently blocking enzyme function, and that the target can thus be used with confidence for developing drug candidates.

\section{Different Targets Available for TB Therapy: Old and New}

Most of the currently used anti-TB drugs were discovered serendipitously by screening compounds for anti-tuberculosis properties [15]. For instance, isoniazid (INH) was discovered after observing the growth inhibiting property of its precursor molecule, nicotinamide. Similarly, pyrazinamide would not have been discovered had it not been tested directly in animals, an approach that would be highly unusual today [16]. Prior to the determination of the sequence of the M.tb genome [17], most of the screening activities in TB research relied on a small number of existing targets and whole cell screening. The determination of the M.tb genome sequence revealed a multitude of potential targets, which at the time was hailed as a great breakthrough. However, no new drug candidates have come from new genomically derived targets due to a variety of reasons. First, validation of a target is time-consuming with many components such as, demonstration of biochemical activity of the enzyme and confirmation of essentiality of the target, which is particularly laborious for M.tb. Secondly, M.tb has a characteristic lipophilic cell wall and intracellular penetration is essential for activity for most targets. Thus, compounds that show activity in cell free biochemical screens often are not active in live bacteria as they cannot penetrate the cell wall. This is particularly true when the enzyme target has a polar active site, such as many important metabolic enzymes that would otherwise be ideal targets. Finally, compounds showing activity in whole cell screening against rapidly growing TB are often not active against persistent forms of TB 
i.e., latent TB infection. Some of the most important targets, old and new, available for TB drug discovery are reviewed briefly in the following section. Detailed reviews on various TB targets are provided elsewhere [24, 32-34].

\section{Cell Wall Biosynthesis}

M.tb has a unique, complex cell wall structure. It consists of three covalently linked macromolecules, peptidoglycan, arabinogalactan, and mycolic acids.

- Enoyl carrier protein reductase (InhA): Enzymes of the mycobacterial fatty acid biosynthesis pathway, the FAS II complex, are responsible for the biosynthesis of mycolic acids [35]. Mycolic acids are branched $\beta$-hydroxy fatty acids composed of an intermediate length (C24-C26) saturated $\alpha$-chain and a longer (>C50) meromycolate chain that contains characteristic functional moieties. Enoyl carrier protein reductase (InhA) is the enzyme of the FAS II complex that catalyzes the final step in the elongation of mycolic acids [18]. The anti-TB drugs INH and ethionamide both act by inhibiting this enzyme, but via different mechanisms. Recently, a new generation of promising biphenyl InhA and Fab I inhibitors have been developed that do not require prodrug activation [36].

- Arabinosyl transferases are responsible for synthesis of the unique arabinogalactan component of mycobacterial cell wall [22]. The anti-TB drug ethambutol acts by inhibiting this pathway. Recently, a novel enzyme, arabinofuranosyl transferase $(A f t A)$, has been identified that catalyzes the addition of the first key arabinofuranosyl residue to the galactan domain of the cell wall, thus priming the galactan for further elongation [37]. This enzyme and others in the arabinogalactan biosynthesis pathway have been found essential for growth and could serve as potential targets for drug discovery [38-40].

- D-alanine racemase (Alr) catalyzes the first committed step in the bacterial peptidoglycan biosynthesis. Peptidoglycan is a structural component of most bacterial species including mycobacteria. The second-line anti-TB drug cycloserine exerts activity by inhibiting this enzyme [29].

\section{Nucleic Acid Synthesis}

- $\quad$ RNA polymerase is responsible for transcribing RNA from DNA. The $\beta$-subunit of this enzyme is the target of the first-line anti-TB drug rifampicin and other drugs in the rifamycins class [20].

- DNA gyrase (Topoisomerase IV) is responsible for unwinding the DNA supercoil for replication and transcription [26]. Drugs belonging to the fluoroquinolone class such as moxifloxacin and ciprofloxacin act by inhibiting this enzyme and show good anti-tuberculosis activity [41]. 


\section{Protein Biosynthesis}

Streptomycin, an aminoglycoside antibiotic, acts by interfering with protein synthesis. The site of action is at ribosomal protein S12 (rpsL) of the 30S subunit of the ribosome, and 16S rRNA (rrs) in the protein synthesis [23]. The other drugs in the class, kanamycin, amikacin and capreomycin also exhibit similar action by inhibiting $16 \mathrm{~S}$ rRNA in protein synthesis [24]. Linezolid, which belongs to the new orally active oxazolidinone class of antibiotics, inhibits protein synthesis at the early stage by binding to the 50S subunit of the 23S ribosomal RNA and has shown promising anti-tuberculosis activity [42].

\section{Co-factor Biosynthesis}

Folate derivatives are cofactors utilized in the biosynthesis of essential molecules such as purines, pyrimidines and amino acids. Bacteria synthesize these folate cofactors via a de novo pathway. Dihydrofolate reductase and dihydropteroate synthase are the two enzymes involved in the folate biosynthesis that have been the targets for antibacterial drug discovery for many years. Para-aminosalicylic acid (PAS), an anti-TB drug, is thought to have activity on dihydrofolate reductase [27, 32].

\section{Miscellaneous Targets}

- Pyrazinamide, a first-line anti-TB drug, exhibits activity only in vivo. It is thought to act by de-energizing the proton motive force of the membrane that affects membrane transport [21].

- TMC207 (R207910), a novel diaryl quinoline analog in development, acts by inhibiting ATP synthase, a novel target for TB drug discovery [43].

- Isocitrate lyase (ICL), an enzyme in the glyoxylate pathway that allows the net synthesis of dicarboxylic acids from $\mathrm{C} 2$ compounds such as acetate, has been found essential for the survival of persistent TB organisms [44] and because of this feature ICL is viewed by many as an important target for future anti-TB drug discovery.

- Other targets that are being explored as novel drug targets include proteins involved in amino acid biosynthesis, regulatory proteins, proteins involved in stringent response, and enzymes involved in terpenoid biosynthesis [32].

\section{Ideal Properties of an Anti-TB Drug}

Desirable properties for a new drug are determined by unmet needs. The objectives of a newly developed antitubercular therapy are threefold [15]:

- The newly developed TB treatment should reduce the duration of therapy and the number of doses needed to be taken per day. 
- The newly developed TB treatment should provide treatment options for multidrug resistant tuberculosis (MDR-TB) and extensively drug resistant tuberculosis (XDR-TB) that are not otherwise treatable with an existing therapy.

- The newly developed TB treatment should provide treatment options for latent TB infections and should be able to eradicate its existence in low-incidence countries. This is an important requirement as one-third of the world's population is currently infected with TB and reactivation to an active TB could occur when the immune system is weakened.

As stated earlier, the in vivo activity of anti-infectives is dictated by their intrinsic antimicrobial activity and their free, unbound concentration in the target tissue, which is determined by its biopharmaceutic and pharmacokinetic properties. Desirable characteristics of a new TB drug from the biopharmaceutic, pharmacokinetic and pharmacodynamic point of view are discussed in the following [15].

\section{Biopharmaceutic Properties}

TB calls for chronic therapy. Accordingly, patient adherence to the dosing regimen is crucial to the success of therapy as well as to prevent emergence of drug resistance. Oral administration is the route of choice for TB drugs due to its ease of administration and good patient adherence. Consequently, high oral bioavailability is imperative for a new TB drug. Aqueous solubility and gastrointestinal permeability are two important prerequisites for high oral bioavailability. Further, the compound should also have sufficient physical and chemical stability to ensure a long shelf-life. In addition, the compound should not have any physical or chemical incompatibility with other TB drugs used in combination regimens.

\section{Pharmacokinetic Properties}

The compound should allow less frequent dosing (once a day or once weekly) than current regimens for improved patient adherence. Accordingly, a new TB drug is required to have a long elimination half-life. In addition, a new TB drug should be devoid of drug interaction potential with the other drugs used in TB as well as drugs used to treat comorbid conditions such as HIV infection. The compound should not have high protein binding, which could limit the free fraction available for drug action.

\section{Pharmacodynamic Properties}

A new TB drug is expected to have activity against actively replicating and persistent populations of mycobacteria in intracellular and extracellular environments. Activity against dormant/persistent bacteria facilitates shortening of the duration of therapy. The compound should provide treatment options for MDR-TB and XDR-TB. The compound should prevent emergence of drug resistance either alone or in 
combination therapy. In addition, the compound should not produce cross-resistance to other antibiotics. This could be achieved provided the compound has a novel mechanism of action. A new TB drug is also expected to treat latent TB (LTB). The compound should have no acute or long-term toxicity. This is an important feature for a new TB drug since TB drugs usually are taken for much longer durations than normal antibiotic therapies. The compound should not produce drug-comorbid disease interactions. Thiacetazone, for example, produces severe cutaneous hypersensitivity reactions in HIV infected populations [45].

\section{Biopharmaceutic, Pharmacokinetic, and Pharmacodynamic Properties of Anti-TB Drugs}

\section{Biopharmaceutics}

Biopharmaceutics is concerned with the relationship between physicochemical properties of drug molecules and its dosage forms on the rate and extent of drug absorption. The most important biopharmaceutical/physicochemical properties that influence the rate and extent of absorption of drugs are solubility and permeability [4648]. Other important parameters include lipophilicity, charge state $(\mathrm{pKa})$ and stability in gastrointestinal fluids $[6,49,50]$.

\section{Solubility}

Solubility is a key property for the absorption of orally administered drugs. A compound is conventionally classified as highly soluble when the largest dose of the compound is soluble in less than $250 \mathrm{~mL}$ water over a $\mathrm{pH}$ range from 1.0 to 7.5. 'Soluble' compounds have a solubility range of greater than or equal to $33 \mathrm{mg} / \mathrm{mL}$. 'Sparingly soluble' compounds have a range from 10-33 mg/mL, 'slightly soluble' compounds from $1-10 \mathrm{mg} / \mathrm{mL}$, and 'very slightly soluble' compounds from $0.1-1 \mathrm{mg} / \mathrm{mL}$. Compounds with solubility below $1 \mathrm{mg} / \mathrm{mL}$ are classified as 'practically insoluble'. Sparingly soluble compounds are frequently difficult to formulate into oral dosage forms that promote the bioavailability of the active ingredient.

\section{Permeability}

Permeability is another key biopharmaceutical property that depicts the ability of a molecule to cross biological membranes, which in turn largely affects its uptake and disposition in the body. The rate and extent of absorption of a drug after oral administration is dependent upon the concentration of drug in the intestinal lumen and permeability of the intestinal mucosa provided the drug is not degraded or metabolized in the gut. Aqueous solubility of above $50 \mu \mathrm{g} / \mathrm{mL}$ is acceptable for a compound that has mid-range permeability and an average potency of $1 \mathrm{mg} / \mathrm{kg}$ in most preclinical drug development programs. pKa, the negative logarithm of acid dissociation constant of a molecule, provides a perspective on the acidic or basic nature of a molecule and is useful 
to calculate the extent of ionization of a molecule in an aqueous environment. In practical terms, the unionized molecule exists for acids $2 \mathrm{pH}$ units below and for bases $2 \mathrm{pH}$ units above its pKa. Degree of ionization affects the lipophilicity of a molecule, and eventually affects permeability and many other physicochemical properties. Amidon et al. proposed a biopharmaceutics classification system (BCS) under which drugs can be categorized into four groups according to their solubility and permeability properties [46]. Drugs with high solubility and high permeability are grouped into class I, low solubility and high permeability into class II, high solubility and low permeability into class III, and low solubility and low permeability into class IV. Drugs falling under classI are considered ideal and those falling under II and III exhibit solubility and permeability limited absorption, respectively. Drugs falling under class IV are not desirable as they are difficult to be developed as orally active molecules. Currently used antitubercular agents are classified according to the BCS in Table 1-2 [46]. Ideally, the classification of drug solubility should be based on the experimental solubility values and permeability classification on the experimental human jejunal permeability data or well-defined mass balance studies. Since the experimental permeability data is not readily available for most of the drugs, permeability classification was done in this review based on the $n$-octanolwater partition coefficient $(\log \mathrm{P})$. Compounds having a dose number $\left(\mathrm{D}_{0}\right) \leq 1\left(\mathrm{D}_{0}=\right.$ maximum dose strength $(\mathrm{mg}) /[250 \mathrm{x}$ solubility $(\mathrm{mg} / \mathrm{mL})])$ are considered as highly soluble and those having an $n$-octanol-water partition coefficient $(\log \mathrm{P})$ values of above 1.72 are considered as highly permeable compounds [51].

\section{Pharmacokinetics}

Pharmacokinetics (PK) describes the time course of absorption, distribution, metabolism and excretion of drug substances in the body. In essence, pharmacokinetics describes 'what happens to a drug molecule in the body' [52]. The utility of pharmacokinetics lies in determining the best way to administer a drug to achieve its therapeutic objective. PK parameters of first-line and second-line anti-TB drugs are given in Table 1-3.

\section{Absorption}

The process of in vivo drug absorption is a complex phenomenon involving several mechanisms. The principal mechanisms of drug absorption include passive diffusion (transcellular and paracellular) and carrier-mediated transport (active transport and facilitated diffusion). Physicochemical properties such as solubility, lipophilicity, charge state (extent of ionization) and stability, and physiological conditions such as gastric emptying time, intestinal transit time and gastrointestinal $\mathrm{pH}$ influence the absorption of drug molecules across the gastrointestinal mucosa. En route to systemic circulation, a drug has also to cross 'metabolic barriers' such as first-pass intestinal and hepatic metabolism. The 'metabolic barriers' coupled with the physicochemical barriers determine the extent of drug absorbed from a given oral dose. 
Table 1-2. Biopharmaceutic Properties of Anti-Tuberculosis Drugs.

\begin{tabular}{|c|c|c|c|c|c|c|c|c|}
\hline Drug & $\mathbf{M W}$ & $\begin{array}{c}\text { Maximum } \\
\text { Dose Strength } \\
\text { (mg) }\end{array}$ & $\begin{array}{l}\text { Aqueous } \\
\text { Solubility } \\
\text { (mg/mL) }\end{array}$ & $\begin{array}{c}\text { Dose Number } \\
\left(D_{0}\right)\end{array}$ & $\log P$ & pKa & $\begin{array}{l}\text { BCS } \\
\text { Class }\end{array}$ & References \\
\hline Isoniazid & 137.1 & 300 & 140 & 0.009 & 0.64 & $\begin{array}{c}2.0,3.6 \text { and } \\
10.8\end{array}$ & 3 & {$[51,53]$} \\
\hline Rifampin & 823.0 & 300 & $0.1\left(2.8^{*}\right)$ & 12 & 3.72 & 1.7 and 7.9 & 2 & {$[51,54,55]$} \\
\hline Pyrazinamide & 123.1 & 400 & $15\left(26.5^{\$}\right)$ & 0.107 & -1.41 & 0.5 & 3 & {$[51,54]$} \\
\hline Ethambutol.2HCl & 277.2 & 400 & 100 & 0.016 & 0.06 & 6.6 and 9.5 & 3 & {$[51,56]$} \\
\hline Streptomycin & 581.6 & 500 & $>20$ & N/A & -8.005 & na & N/A & {$[51,54,57]$} \\
\hline Kanamycin & 484.5 & 500 & Freely soluble & N/A & -7.936 & na & N/A & {$[51,54,58]$} \\
\hline PAS & 153.1 & 4000 & 1.67 & 9.58 & 1.012 & 2.015 & 4 & {$[51,54,59]$} \\
\hline Cycloserine & 102.1 & 500 & 100 & 0.02 & -1.631 & na & 3 & {$[51,54,60]$} \\
\hline Ethionamide & 166.2 & 500 & $\begin{array}{l}\text { Practically } \\
\text { insoluble }\end{array}$ & $>20$ & 0.705 & na & 4 & {$[51,54]$} \\
\hline Ciprofloxacin. HCl & 331.3 & 750 & 10 & 0.3 & 1.335 & 6.09 & 3 & {$[51,54]$} \\
\hline Ofloxacin & 361.4 & 400 & 28.3 & 0.0056 & 1.268 & na & 3 & {$[51,54]$} \\
\hline Linezolid & 337.3 & 600 & 3 & 0.8 & 0.232 & na & 3 & {$[51,54]$} \\
\hline Moxifloxacin & 401.4 & 400 & na & na & 2.033 & na & na & {$[51,54]$} \\
\hline Gatifloxacin & 375.4 & 400 & $40-60$ & $0.04-0.027$ & 1.801 & na & 1 & {$[51,54]$} \\
\hline Metronidazole & 171.2 & 500 & 10 & 0.2 & -0.262 & na & 3 & {$[51,54]$} \\
\hline
\end{tabular}

* Solubility at $\mathrm{pH} 7.5$.

$\$$ Solubility at temperature $38^{\circ} \mathrm{C}$.

N/A: Not applicable (administered by intramuscular injection).

Dose number $\left(\mathrm{D}_{0}\right)$ is calculated using the formula, $\mathrm{D}_{0}=$ Maximum dose strength $(\mathrm{mg}) /[250(\mathrm{~mL}) *$ solubility $(\mathrm{mg} / \mathrm{mL})]$. LogP: $n$-Octanol-water partition coefficient. 
Table 1-3. Pharmacokinetic Parameters of Anti-Tuberculosis Drugs.

\begin{tabular}{|c|c|c|c|c|c|c|c|c|c|c|}
\hline Drug & $\begin{array}{l}\text { Dose } \\
\text { (mg) }\end{array}$ & $\mathbf{F}$ & $\begin{array}{c}\mathbf{t}_{1 / 2} \\
(\mathbf{h r})\end{array}$ & $\begin{array}{l}\text { Protein } \\
\text { Binding }\end{array}$ & $\begin{array}{l}\mathbf{T}_{\max } \\
(\mathbf{h r})\end{array}$ & $\underset{(m g / L)}{C_{\max }}$ & $\begin{array}{c}\text { Clearance } \\
(\mathrm{L} / \mathrm{hr})\end{array}$ & $\begin{array}{l}\text { Volume of } \\
\text { Distribution } \\
\text { Vd (L) } \\
\end{array}$ & $\begin{array}{c}\text { \%Excreted } \\
\text { Unchanged } \\
\text { in Urine }\end{array}$ & References \\
\hline Isoniazid & 300 & - & $\begin{array}{c}2-4.5 \text { (Slow) } \\
0.75-1.8 \text { (Fast) }\end{array}$ & Negligible & $1-2$ & $3-5$ & $\begin{array}{c}22.7-39.5^{\mathrm{a}} \\
11-20.2^{\mathrm{a}}\end{array}$ & $36.4-57.4^{\mathrm{a}}$ & $\begin{array}{c}5-9^{\#} \\
24-34^{\#}\end{array}$ & [61-63] \\
\hline Rifampicin & 600 & $90-95 \%$ & $2-5$ & $60-90 \%$ & $1.5-2$ & $3-10$ & $8-21.42^{b}$ & 55 & 4-10 & {$[31,61-65]$} \\
\hline Ethambutol & 800 & $70-80 \%$ & $3-4$ & $6-30 \%$ & $2-4$ & $2-5$ & $32.8-39.5$ & $19-21$ & $75-80$ & [61-63] \\
\hline Pyrazinamide & 1000 & $>70 \%$ & $10-24$ & $5-10 \%$ & 2 & $45-65$ & $0.84-9.66^{\mathrm{c}}$ & $40-52^{c}$ & $4-14$ & [61-63] \\
\hline Ethionamide & 250 & $>90 \%$ & $2-3$ & $30 \%$ & $1-3$ & 2.16 & na & $93.5^{\mathrm{d}}$ & 1 & [61-63] \\
\hline Thiacetazone & 150 & na & 4 & $\sim 95 \%$ & $4-5$ & $1.6-3.2$ & na & na & 42 & [61-63] \\
\hline PAS & $\begin{array}{c}\text { Free Acid } 4000 \\
\text { Sodium Salt } \\
4000\end{array}$ & na & $2-3$ & $15 \%$ & $\begin{array}{c}3-4 \\
0.5-1\end{array}$ & $\begin{array}{l}41-68 \\
76-104\end{array}$ & na & 7.4 & 30 & [61-63] \\
\hline Cycloserine & 750 & $70-90 \%$ & 10 & na & $3-4$ & $20-35$ & na & $8-18$ & 70 & {$[61-63]$} \\
\hline Streptomycin* & 1000 & - & $2-4$ & $35-57 \%$ & $<1$ & $50-60$ & na & $76-115$ & 90 & [61-63] \\
\hline Kanamycin* & 1000 & - & $2-3$ & Negligible & $<1$ & $20-35$ & na & $13-28$ & 99 & [62] \\
\hline Rifapentine & 600 & na & $14-18$ & $98 \%$ & $5-6$ & $8-30$ & na & na & $<10$ & {$[61,64]$} \\
\hline Ciprofloxacin & 750 & $70 \%$ & 4 & $20-40 \%$ & $1-2$ & 4.3 & $18^{\mathrm{e}}$ & 260 & $40-50$ & {$[66,67]$} \\
\hline Ofloxacin & 600 & $85-95 \%$ & $3-7$ & $20-30 \%$ & $1-2$ & 10 & na & 90 & 75 & [66-68] \\
\hline Moxifloxacin & 400 & $86-92 \%$ & $12-14$ & $26-50 \%$ & $1-3$ & $2.5-5$ & $9.1-11.6$ & 119-189 & 20 & {$[66,69]$} \\
\hline Gatifloxacin & 400 & $96 \%$ & $7-14$ & $20 \%$ & $1-2$ & $2.33-3.59$ & 11.4 & 126 & $>70$ & {$[66,70]$} \\
\hline Linezolid & 600 & $\sim 100 \%$ & $4.5-5.5$ & $31 \%$ & $1-2$ & 9-16 & $6-12$ & $40-50$ & 30 & [71] \\
\hline
\end{tabular}

*Streptomycin and kanamycin are administered intramuscularly.

\# Includes unchanged drug and metabolites (hydrazones).

a $\mathrm{V}_{\mathrm{ss}} / \mathrm{F}$ and $\mathrm{CL} / \mathrm{F}$ reported.

$\mathrm{b} \mathrm{CL} / \mathrm{F}$.

c $\mathrm{V}_{\text {area }} / \mathrm{F}$ and $\mathrm{CL} / \mathrm{F}$ reported.

$\mathrm{d} V / F$ reported.

e $\mathrm{CL}_{\text {renal }}$ reported. 


\section{Distribution}

Once drug has entered the vascular system, it gets distributed throughout the various tissues and body fluids. However, most drugs do not distribute uniformly and similarly throughout the body. The differences in tissue distribution of drugs can be attributed to differential tissue affinity, blood flow to tissues, ability to cross biomembranes and physicochemical properties (lipophilicity and extent of ionization). Many drugs interact with body components such as proteins and other macromolecules. Binding to protein structures in the plasma is an important binding process for many drugs, and plasma protein binding is a major determinant of drug disposition. It is a well known fact that the drug bound to protein is not pharmacologically active, and is oftentimes not accessible to drug metabolizing enzymes (e.g. in the liver) and excretion processes of organs such as kidneys and lungs. Furthermore, binding to proteins also restricts the mobility of drug across biomembranes. On the contrary, free drug is relevant for the pharmacological effects as it can interact with pharmacologic target structures, can be metabolized and excreted, and may pass biomembranes. Thus, only free drug concentrations are of therapeutic interest.

\section{Metabolism}

Metabolism/biotransformation and excretion are the two major pathways of drug elimination from the body. In general, metabolizing enzymes convert drugs into more polar molecules that can easily be excreted by elimination organs such as the kidneys. Usually, metabolism results in immediate loss of activity due to changes in lipophilicity, polarity etc. that hinders a drug's access to its site of action. However, biotransformation makes some drugs more potent than the parent compounds (bioactivation). Metabolism takes place by enzymatic catalysis, predominantly in the liver, but may also occur in other body organs and tissues such as plasma, kidneys, lungs and intestines. Diverse classes of enzymes are available for elimination of drugs in the body. Based on the proportion of drugs and endogenous molecules metabolized, the most important and the best-characterized class of drug-metabolizing enzymes is the cytochrome P450 (CYP) family [72]. It is involved in the oxidative metabolism of many endogenous chemicals, as well as herbal components and drug molecules.

\section{Excretion}

Excretion is referred to as the irreversible removal of drug from the body. Although the kidneys are the major organ of drug excretion, drug molecules are also excreted from several other organs such as the lungs, the intestines, the skin, and the liver via the bile. The major mechanisms of drug excretion in the kidneys include glomerular filtration and active tubular secretion. Drugs with small molecular weight are removed by glomerular filtration. Their removal, however, is limited to only the free fraction of drug in the plasma. Physicochemical parameters such as lipophilicity, solubility, urinary $\mathrm{pH}$ and ionization status of a molecule play a role in the elimination by the kidneys. Lipophilic drugs get frequently reabsorbed by passive diffusion processes summarized as 
tubular reabsorption. Thus the tubular reabsorption process limits drug excretion by the kidneys.

Tubular secretion in the kidneys as well as biliary secretion in the liver are active transporter-mediated elimination processes. Active drug transport across biomembranes has recently been identified playing a major role in drug absorption, distribution and elimination processes. The human MDR1 gene product, p-glycoprotein, has been described as a major determinant of pharmacokinetics of numerous drugs. Other transporters such as multidrug resistant proteins in the liver, MRP1 and MRP2, are also involved in the elimination of numerous drug molecules and their metabolites. In the kidneys, active tubular secretion is largely mediated by a series of organic cation (OCT) and anion (OAT) transporters.

A brief discussion of key PK parameters is provided in the following for a better understanding of their implications in drug development and pharmacotherapy:

\section{Bioavailability $(F)$}

Oral bioavailability of drugs is defined as rate and extent to which a drug is absorbed and becomes available in the systemic circulation. Extent of oral bioavailability $(F)$ of a drug molecule is expressed as the fraction of the orally administered dose relative to an intravenous dose that gets absorbed into the systemic circulation. $F$ ranges from 0 ( $0 \%$ absorption) to 1 (100\% absorption). The absolute oral bioavailability $(F)$ is of great interest as it determines the dose to maintain concentrations sufficient for effective pharmacotherapy.

\section{Clearance $(C L)$}

Clearance is a measure of drug removal efficiency from the body. In general, more than one organ contributes to the elimination of drug from the body and total clearance of a drug is the sum of all elimination organ clearances. Clearance is a fundamental pharmacokinetic property. It determines exposure to a given drug dose and consequently determines (together with $F$ ) the dose of a drug to achieve effective pharmacotherapy. Also, clearance is a determinant of the elimination half-life of a drug and consequently, determines together with volume of distribution the frequency of drug administration.

\section{Volume of Distribution $\left(V_{d}\right)$}

The volume of distribution relates the amount of drug in the body to the plasma drug concentration. The magnitude of $\mathrm{V}_{\mathrm{d}}$ indicates whether the drug is residing largely within or outside the vascular space. $\mathrm{V}_{\mathrm{d}}$ is also a fundamental pharmacokinetic property. Together with clearance, it determines elimination half-life of the drug and consequently, frequency of drug administration. 


\section{Half-life $\left(t_{1 / 2}\right)$}

The elimination half-life $\left(t_{1 / 2}\right)$ of a drug is the time required for elimination of half of the bioavailable dose. It is a derived pharmacokinetic parameter and is determined by the two fundamental parameters of drug disposition, clearance and volume of distribution.

$$
t_{1 / 2}=\frac{0.693 \times V_{d}}{C L}
$$

The elimination half-life of a drug determines the frequency of drug administration. Together with the extent of bioavailability $(F)$ and clearance $(C L)$, it determines the dosage regimen for effective pharmacotherapy.

\section{Pharmacodynamics}

Pharmacodynamics (PD) is defined as the intensity of a drug effect in relation to its concentration in the body. In general, pharmacodynamics is what the drug does to the body [52]. However, antimicrobial pharmacodynamics is what the drug does to the pathogen residing in the host organism.

\section{Measures of Anti-Tuberculosis Activity}

The minimum inhibitory concentration (MIC) and the minimum bactericidal concentration (MBC) have been the major pharmacodynamic parameters used to quantify the activity of an antimicrobial agent against the infecting pathogen. The MIC is defined as the lowest drug concentration that inhibits growth of microorganisms after 16-20 h of incubation with a standard inoculum $\left(1-5 \times 10^{5} \mathrm{CFU} / \mathrm{mL}\right)$ [73]. The $\mathrm{MBC}$ is defined as the drug concentration that produces $99.99 \%$ killing of the initial inoculum. The MIC and the $\mathrm{MBC}$ are in vitro measures of antimicrobial's efficacy. In vivo measures of antitubercular activity include early bactericidal activity (EBA) and sterilizing activity of a drug. EBA is defined as the rate of fall of colony forming units $(\mathrm{CFU} / \mathrm{mL})$ in the sputum of patients during the first two days of drug treatment and is expressed as $\log _{10}$ $(\mathrm{CFU} / \mathrm{mL}) /$ day. The EBA of a drug reduces the viable counts of bacteria in the patient's sputum rapidly thereby rendering them noninfectious. Sterilizing activity of a drug is defined as the ability to sterilize the sputum, caseous granulomas in the lungs and the spleen and prevent relapse, i.e., it captures activity against slow growing and persister populations of mycobacteria which are responsible for relapse.

\section{In vivo Models of Anti-Tuberculosis Activity}

For the assessment of in vivo efficacy, several animal models of TB have been established in a variety of species, including mice, guinea pig, rabbit and non-human primates. The choice of animal model must be weighed against the feature of the disease captured by the model and careful interpretation of the data obtained from each model is 
warranted. Detailed reviews on various animal models of TB can be found elsewhere [7477].

The availability of a variety of genetically defined mouse strains as well as their ease of manipulation, housing and their low cost made murine models of TB most popular among the available models. However, the pathology of disease in murine models is markedly different from humans. After aerosol infection, the granulomas developed in the lungs are not well-formed structures as opposed to human disease. Caseous necrosis and cavity formation that are characteristic of human M.tb infection are not observed in the mouse model of TB. During the active TB infection, human lung contains an array of granulomas (solid, caseous, necrotic and cavitary) with varying numbers of bacteria. In contrast, little or no such heterogeneity is observed in mouse lung. During the chronic phase of the disease, unlike humans, high numbers of bacteria are found in the lungs and the spleen $[74,76]$. Thus, the mouse model is not ideal for studying latent TB. However, murine models can be used for evaluation of EBA of novel drug compounds and new dosing regimens.

Recently, interferon- $\gamma$ (IFN- $\gamma$ ) knockout mouse models have been utilized for rapid screening of the efficacy of new drug compounds against TB. Without the protective IFN- $\gamma$ gene, these mice are highly susceptible to the M.tb infection and, therefore, the activity of a compound can be seen rapidly when compared to untreated controls. This sensitive mouse model requires only 9 days of treatment and a small number of animals, which makes it an ideal model for first line testing of TB compounds [78]. Wayne showed in his in vitro model of dormancy that the oxygen depleted M.tb cultures enter a non-replicating persistence state [79]. Recently, it has been shown that animals infected intranasally with oxygen depleted cultures can be used to test against TB vaccines [80]. Once optimized, this model also may be used to screen the activity of candidate drugs against persistent forms of TB.

The formation of granulomas and associated caseation in rabbit and guinea pig models of TB is closely similar to human disease. Hematogenous spread to uninfected lobes occurs in both models. The exponential increase in bacterial numbers in the lungs and caseous necrosis during cell mediated immunity in guinea pigs ultimately progress to fatal disease within a time frame of several months to a year. Persistent populations of bacteria have been observed in the primary lesions. Thus, the guinea pig model may be useful to screen new drug compounds against persistent forms of TB [81]. Since rabbits are more resistant to M.tb infection and highly susceptible to Mycobacterium bovis, much of the research in rabbits has been carried out using M. bovis. Caseation is moderate, and cavity formation and bronchial dissemination are observed in rabbit lungs $[74,76]$.

TB in non-human primates is markedly similar to human disease and exhibits antigen induced T-lymphocyte activity both in vitro and in vivo. Although expensive, it is the best currently available model of latent TB [74, 76]. Since shortening of duration of TB therapy is largely dependent on eradicating the latent disease, the non-human primate model is probably the best model for studying the efficacy of new drugs and combination treatment regimens. 


\section{First-Line Agents}

\section{Isoniazid}

Isoniazid (INH) is highly active against $M . t b$ with an MIC of $0.01-0.25 \mu \mathrm{g} / \mathrm{mL}$. It is bactericidal against rapidly dividing bacteria, but has limited activity against slow growing populations. INH is a prodrug that requires activation by the catalase $(\mathrm{kat} G)$ gene of M.tb $[82,83]$. The action of INH is quite complex and involves several targets [84], but the most significant is InhA causing interference with the synthesis of mycolic acids, an essential component of the mycobacterial cell wall [85]. Drug resistance to INH is commonly mediated via mutations in the kat $G$ gene, leading to the loss of catalaseperoxidase activity and the failure to produce toxic INH derivatives [82]. INH is known to cause hepatotoxicity in a small number of patients, with an increased risk for individuals with a slow acetylator phenotype.

INH is highly hydrophilic in nature and has a high, temperature-dependent water solubility of $14 \mathrm{~g}$ in $100 \mathrm{~mL}$ at $25^{\circ} \mathrm{C}$ and $26 \mathrm{~g}$ in $100 \mathrm{~mL}$ at $40^{\circ} \mathrm{C}$ [53]. The aqueous solubility of INH is highly favorable for oral absorption. There is a discrepancy in the literature on the acid dissociation constants $(\mathrm{pKa})$ of INH. This is in part due to the difference in measurement methods employed. INH has three different $\mathrm{pKa}$ values, $\mathrm{pKa} 1$ $=2.13, \mathrm{pKa} 2=3.81$ and $\mathrm{pKa} 3=11.03$ [86]. In another report, $\mathrm{pKa}$ values were found to be $2.00,3.6$, and 10.8 for $\mathrm{pKa} 1, \mathrm{pKa} 2$ and $\mathrm{pKa} 3$, respectively [87]. INH exists in an ionized state in the entire $\mathrm{pH}$ range of the gastrointestinal tract. The hydrophilicity of INH is further reflected by its low $n$-octanol-water partition coefficient of 0.64. [51]. Hydrophilicity, low- $n$-octanol water partition coefficient and charge state, however, result in a relatively low permeability of INH. Thus, INH can be classified under BCS as a class-III drug, i.e., a compound with high solubility and low permeability [51]. However, this classification is based on the correlation between partition coefficients and permeability of the compounds and such correlations have only limited predictability. The data on INH oral absorption and permeability are rather inconclusive for its BCS classification, but it suggests INH to be on the borderline of BCS class-I and class-III [88]. As with the other BCS class-III drugs, oral absorption of INH is decreased by food.

Following oral administration, INH undergoes rapid and complete absorption with peaks occurring 1-2 hours after drug intake. Absorption of INH is decreased by food and antacids. INH is distributed extensively into tissues and body fluids including CSF. It passes through the placental barrier and is secreted into milk achieving concentrations comparable to plasma. Protein binding of the drug is negligible. It is metabolized in the liver primarily by acetylation and dehydrazination. The acetylation of INH is mediated by a polymorphically expressed enzyme, N-acetyl transferase 2 (NAT2). As a result, the population can be divided as slow acetylators and fast acetylators based on their NAT2 metabolizing capacity. The majority of Asians and about $50 \%$ of Caucasians and Blacks are rapid acetylators. However, the rate of elimination does not significantly alter the effectiveness of the drug. 5-30\% of the orally administered drug is excreted in the urine, 
mostly as metabolites. Higher percentages have been observed after IV administration suggesting significant first-pass metabolism [62, 89-91].

\section{Rifampicin}

Rifampicin (RIF) is the mainstay of current anti-tuberculosis therapy. Chemical structure of RIF and other established anti-TB drugs are shown in Figure 1-1. RIF shows bactericidal activity against both actively growing and slowly metabolizing non-growing bacilli $[24,83]$. RIF in concentrations of $0.005-0.2 \mu \mathrm{g} / \mathrm{mL}$ inhibits the growth of M.tb in vitro. RIF's activity against dormant forms of mycobacteria is thought to be responsible for its effect of reducing the duration of TB therapy necessary for sterilization [92]. Resistance to RIF is thought to occur primarily through changes in the $r p o B$ gene leading to alteration of the target site on RNA polymerase [93]. Resistance to RIF has been reported to also confer cross-resistance to other rifamycins such as rifabutin and rifapentine. RIF's metabolite, 25-desacetyl rifampin, retains most of RIF's activity. Frequent adverse effects of RIF include skin rash, fever and GI distress. Allergic reactions also have been reported in the treatment with RIF and occur more frequently during high dose treatment (>900 mg twice weekly) $[83,89,94]$.

Analogous to INH, RIF also exhibits $\mathrm{pH}$ and temperature dependent water solubility. At room temperature RIF has a solubility of $2.8 \mathrm{mg} / \mathrm{mL}$ at $\mathrm{pH} 7.5$ and 99.5 $\mathrm{mg} / \mathrm{mL}$ at $\mathrm{pH} 2.0$ [55]. At $37^{\circ} \mathrm{C}$, RIF shows a solubility of $200 \mathrm{mg} / \mathrm{mL}$ in $0.1 \mathrm{~N} \mathrm{HCl}$ and $9.9 \mathrm{mg} / \mathrm{mL}$ in phosphate buffer $\mathrm{pH} 7.4$ [95]. RIF is very stable in solid state in sealed containers at room temperature. In contrast, it shows decreased stability in aqueous solutions. It undergoes desacetylation at alkaline $\mathrm{pH}$ and transforms into a quinone in the presence of oxygen. RIF has two pKa values, 1.7 and 7.9. It has an $n$-octanol-water partition coefficient of 3.72 and is classified as a BCS class II drug, i.e., a low solubility and high permeability drug [51].

RIF is well absorbed after oral administration (oral bioavailability of 90-95\%) with therapeutic concentrations occurring in the body 2-4 hours after drug intake. Food interferes with the absorption of rifampicin resulting in an approximate $30 \%$ reduction in the bioavailability. RIF is widely distributed in the body and is about $80 \%$ bound to plasma proteins. RIF is distributed readily into cavity lining, lung parenchyma and kidneys. In contrast, distribution into caseous tissue and cerebrospinal fluid (CSF) are much poorer [96]. Though the distribution into CSF is poorer than into lung tissue, RIF still achieves therapeutic concentrations in CSF. Distribution into CSF is enhanced in local inflammatory conditions such as meningitis [62, 89]. RIF may discolor body fluids such as urine and tears, producing an orange-red color. RIF is metabolized in the body to a desacetylated derivative, which is also active. Importantly, RIF induces the activity of the phase-I drug metabolizing enzymes CYP3A, CYP1A2 and CYP2C [97, 98], the phase-II enzymes glucuronosyl transferases and sulfotransferases [98, 99], and efflux transporter such as p-glycoprotein [100]. It has also been shown to induce the activity of CYP2D6 in individuals with extensive metabolizer phenotype for this enzyme [101]. In addition, RIF induces hepatic enzymes that accelerate its desacetylation. As a result, the 


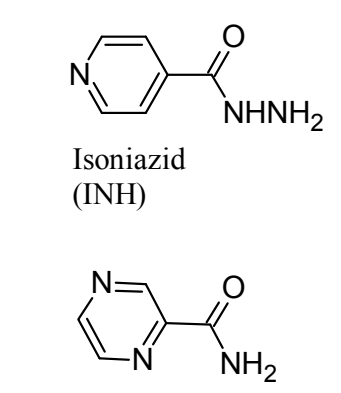

Pyrazinamide (PZA)

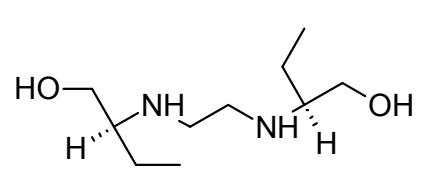

Ethambutol (EMB)

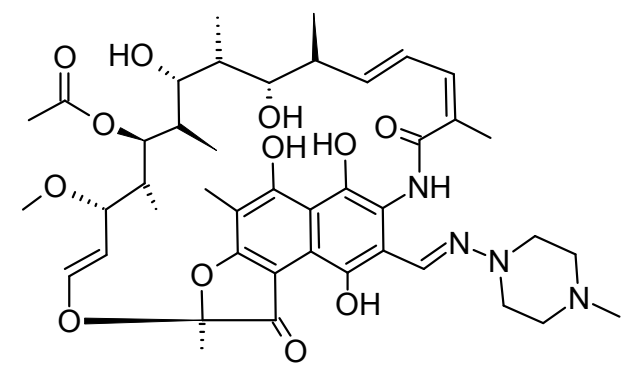

Rifampicin

(RIF)

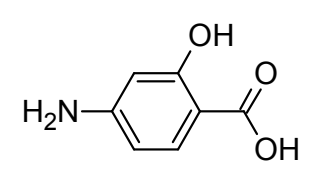

p-Aminosalicylic acid (PAS)

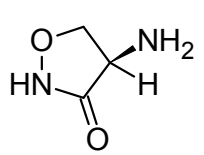

Cycloserine

(CS)

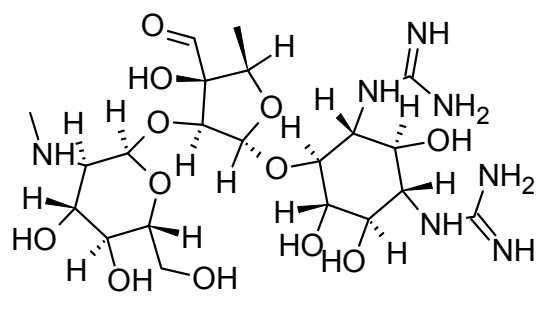

Streptomycin

(SM)

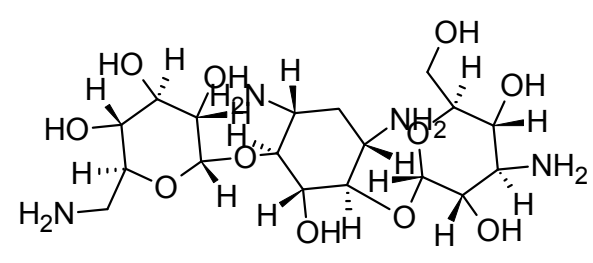

Kanamycin

(KM)

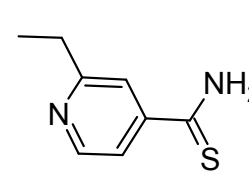

Ethionamide (ETH)

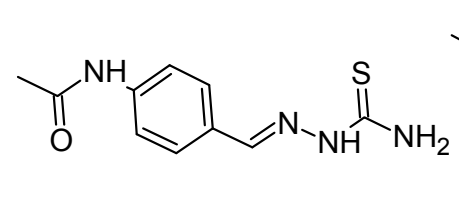

Thiacetazone

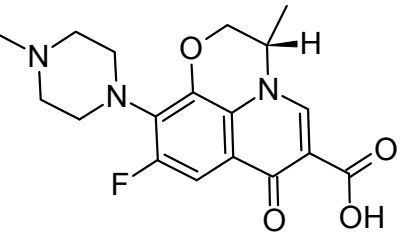

Ofloxacin (OFL)

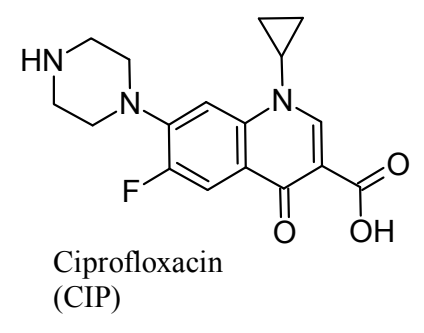

Figure 1-1. Chemical Structures of Established Anti-Tuberculosis Drugs. 
half-life of RIF is progressively shortened by about $40 \%$ during the first 14 days of treatment. However, RIF is not metabolized by CYP metabolism. RIF and its metabolites are rapidly excreted into the bile and undergo enterohepatic circulation. Intestinal reabsorption of desacetylated derivatives, however, is poor and about $65 \%$ of the drug is excreted in the feces. About $35 \%$ of the drug is excreted in the urine, with approximately half of this being unchanged drug [62].

\section{Pyrazinamide}

Pyrazinamide (PZA) is a structural analog of nicotinamide used exclusively for TB therapy. PZA is a paradoxical drug that does not show any activity against mycobacteria under normal culture conditions at neutral $\mathrm{pH}$ but shows high in vivo sterilizing activity [102]. PZA shows in vitro activity only in acidic cultures [103, 104]. Activity of PZA at acidic $\mathrm{pH}$ makes it ideal drug for killing tuberculosis residing in acidic phagosomes within infected macrophages [105]. Addition of PZA to a combination pharmacotherapy against tuberculosis for the first two months reduces the treatment duration by at least 6 months [92]. PZA is a prodrug requiring conversion to its active form, pyrazinoic acid (POA) by the enzyme pyrazinamidase/nicotinamidase in mycobacteria $[24,106]$. A mutation in $p n c A$ that encodes pyrazinamidase is thought to be the mechanism of resistance to PZA in some $M . t b$ strains [106, 107]. Most commonly observed adverse effects of PZA are gastrointestinal distress, arthralgias and elevations in the serum uric acid levels. Hepatotoxicity is the major limiting toxicity for treatment with PZA [83].

PZA, a hydrophilic drug, exhibits temperature dependent water solubility. It shows solubility of $0.64 \mathrm{~g} / 100 \mathrm{~g}$ of solution at $0^{\circ} \mathrm{C}$ and $2.65 \mathrm{~g} / 100 \mathrm{~g}$ solution at $37^{\circ} \mathrm{C}$ [108]. PZA exhibits good stability in the solid state, in both wet or dry atmosphere and daylight. PZA has a reported $\mathrm{pKa}$ value of 0.5 and exists in the ionized state in the gastrointestinal tract. Hydrophilicity of PZA is reflected by its low $n$-octanol-water partition coefficient of -1.41. PZA is classified as a BCS class-III drug, i.e., a high solubility, low permeability drug [51].

PZA is readily absorbed after oral administration with peak concentrations occurring two hours after drug administration. PZA is widely distributed throughout the body. It has excellent tissue penetration in CNS, lungs and liver and achieves high levels in the cerebrospinal fluid as well. Plasma protein binding of the drug is about $10 \%$. PZA is metabolized in the body by hydrolysis followed by hydroxylation to 5hydroxypyrazinoic acid. PZA has a relatively long elimination half-life of 9-10 hours, and metabolites are excreted by the kidneys. The plasma half-life may be prolonged in patients with impaired hepatic or renal function. About $4-14 \%$ of the drug is excreted unchanged in urine $[62,89,90]$. 


\section{Ethambutol}

Ethambutol (EMB) is used as a first-line agent replacing para-aminosalicylic acid due to its better patient tolerability. EMB is active against all forms of mycobacteria but it is generally bacteriostatic. EMB is used in combination regimens while awaiting susceptibility data to INH, RIF and PZA. Use of EMB can be stopped provided the organism is susceptible to the three first-line drugs [83]. EMB interferes with the biosynthesis of a mycobacterial cell wall polysaccharide, arabinogalactan [109]. The enzyme arabinosyl transferase, encoded by embB, is thought to be the major target of EMB [110]. In M.tb, embB is present in an operon along with two other genes, $e m b C$ and $e m b A$ in the order of $e m b C A B$. These three genes share more than $65 \%$ amino acid sequence homology with each other [110]. Mutations in the operon embCAB are thought to be responsible for resistance to EMB [110]. The major adverse effect with EMB treatment is reversible retrobulbar neuritis [83].

EMB is available as dihydrochloride salt and free base. The free base is sparingly soluble in water and shows relatively good solubility in organic solvents [111]. On the contrary, the dihydrochloride salt is readily soluble in water $(100 \mathrm{mg} / \mathrm{mL})$ and dimethyl sulfoxide and is sparingly soluble in organic solvents [56]. EMB is a weak base with reported $\mathrm{pKa}$ values of 6.6 and 9.5. EMB is also a hydrophilic drug and exists in the ionized state throughout the gastrointestinal tract. The hydrophilicity of EMB is further reflected by its low $n$-octanol-water partition coefficient of 0.06 . Based on $\log P$ and aqueous solubility, EMB can be classified as a BCS class-III drug, i.e., a high solubility and low permeability drug [51].

EMB is absorbed rapidly after oral administration with an absolute oral bioavailability of $70-80 \%$. Peak plasma concentrations occur 2-4 hours after drug intake and the drug is well distributed throughout the body and is only about $30 \%$ bound to plasma proteins. EMB does not enter CSF, limiting its use in CSF infections. Approximately $15 \%$ of the drug is metabolized to aldehyde and dicarboxylic acid metabolites. EMB has an elimination half-life of 3-4 hours and the majority of the drug (75-80\% of bioavailable dose) is excreted unchanged in urine. The plasma half-life may be prolonged in renal insufficiency, requiring dosage adjustment. $20-22 \%$ of the drug is excreted unchanged into the feces $[62,90,105,112]$.

\section{Second-Line Agents}

\section{Streptomycin}

Streptomycin (SM) is an aminoglycoside antibiotic produced from Streptomyces griseus. Concentrations ranging from $0.4-10 \mu \mathrm{g} / \mathrm{mL}$ inhibit the growth of the vast majority of strains of M.tb. SM is often used as an additional drug while awaiting susceptibility test results for hospitalized patients and also used as important treatment for some MDR-TB and XDR-TB strains [83]. Streptomycin was shown to cause ototoxicity 
and nephrotoxicity on chronic use. Nephrotoxicity caused by SM is mild and reversible compared with other aminoglycoside antibiotics but administration is often painful and poorly tolerated by patients [83].

SM is available as free base and sulfate, hydrochloride and calcium chloride salt forms. All forms are very soluble in water $(>20 \mathrm{mg} / \mathrm{mL})$ and practically insoluble in chloroform and ether [57].

Because of its polycationic nature SM is not absorbable after oral administration. Thus, it needs to be given by intramuscular injection or intravenously. The dose of SM is $15 \mathrm{mg} / \mathrm{kg}$ per day for patients with normal renal function. It is typically administered as $1000 \mathrm{mg}$ single daily dose or $500 \mathrm{mg}$ twice daily dose, resulting in peak serum levels of about $50-60 \mu \mathrm{g} / \mathrm{mL}$ and $15-30 \mu \mathrm{g} / \mathrm{mL}$, respectively, within one hour of intramuscular administration. SM is widely distributed into body tissues and fluids, passes through the placental barrier and is secreted into milk. However, distribution of SM into the CSF is limited. About $35-57 \%$ of the drug is protein bound. The majority of the drug undergoes renal elimination $(\sim 90 \%)$ and no metabolites have been identified. SM has an elimination half-life of about 2-4 hours. The elimination is dependent on renal function, and dosage adjustment is necessary in case of renal insufficiency [61, 62, 105]

\section{Para-Aminosalicylic Acid}

Para-aminosalicylic acid (PAS), a structural analog of para-amino benzoic acid, is bacteriostatic in nature. It inhibits the growth of M.tb in vitro at a concentration of 1 $\mu \mathrm{g} / \mathrm{mL}$. The antimicrobial activity of PAS is highly specific. Gastrointestinal disturbances are the most commonly observed adverse effects with PAS therapy. It is also reported to produce hypersensitivity reactions and rarely, hepatitis [31, 83].

PAS exits as free acid and as a sodium salt. PAS shows a solubility of $1 \mathrm{~g}$ in 600 $\mathrm{mL}$ water [59]. PAS has a reported $\mathrm{pKa}$ value of 2.015 and exists in the ionized state throughout the gastrointestinal tract. The $n$-octanol-water partition coefficient of PAS was reported as 1.012. Based on this partition coefficient and aqueous solubility, PAS can be classified as a BCS class-IV drug, i.e., a low solubility and low permeability drug.

PAS is readily absorbed after oral administration with peak plasma concentrations occurring within 2 hours after drug intake. A single oral dose of $4 \mathrm{~g}$ of the free acid results in peak serum concentrations of $41-68 \mu \mathrm{g} / \mathrm{mL}$, whereas $4 \mathrm{~g}$ of sodium salt results in peak serum concentrations of 76-104 $\mu \mathrm{g} / \mathrm{mL}$. PAS is well distributed throughout body while distribution into CSF is low. PAS is metabolized by acetylation and $>50 \%$ of drug is acetylated. In contrast to INH, the acetylation of PAS is mediated by $\mathrm{N}$-acetyl transferase 1 (NAT1), for which in contrast to NAT2 genetic variants with defective function are relatively rare [113]. In addition to acetylation, a N-glycyl derivative of PAS is observed in the plasma. When PAS is concomitantly administered with INH the in vivo acetylation of PAS competes with the acetylation of INH, resulting in higher plasma concentrations of free INH than when INH is given alone. This is believed to be due to 
the competition for coenzyme A by the acetylating enzyme systems. However, this fact has no clinical relevance. The drug has an elimination half-life of 2-3 hours and more than $80 \%$ is excreted unchanged in the urine. Elimination of the drug is retarded in case of renal impairment $[30,31,62,90,105]$.

\section{Kanamycin}

Kanamycin (KM) is an aminoglycoside antibiotic produced by Streptomyces kanamyceticus. KM is comprised of three components, Kanamycin A, B and C. Kanamycin $\mathrm{A}$ is the major component of all and designated as kanamycin. KM shows inhibitory activity against $M . t b$ at concentrations of about $6 \mu \mathrm{g} / \mathrm{mL}$ in vitro. $\mathrm{KM}$ is more active at alkaline $\mathrm{pH}$ and exerts a rapid bactericidal effect at concentrations little above the MIC. Like other aminoglycosides, KM inhibits protein synthesis. KM has poor patient tolerability due pain associated with its injection. Minor side effects similar to those encountered with streptomycin have been described with KM therapy. Eosinophilia in the absence of other manifestations of allergy occurs in about $10 \%$ of treated individuals. The most important toxic effect with KM therapy is nephrotoxicity [31].

KM exists as free base, mono sulfate form and acid sulfate form. All forms are soluble in water and almost insoluble in organic solvents. The mono sulfate form shows a solubility of $350 \mathrm{mg} / \mathrm{mL}$ whereas the acid sulfate shows a solubility of 1 part in 1 part of water [58]. KM is highly hydrophilic in nature and has a $\operatorname{logP}$ value of -7.936. Since KM is only administered intramuscularly, BCS classification is not applicable.

Like streptomycin, $\mathrm{KM}$ is poorly absorbed from the gastrointestinal tract. Less than $1 \%$ of the dose is absorbed after either oral or rectal administration. The drug is administered via deep intramuscular injection and is rapidly absorbed from the intramuscular site of injection. An intramuscular dose of $7.5 \mathrm{mg} / \mathrm{kg}$ of $\mathrm{KM}$ results in peak serum levels of $20-35 \mu \mathrm{g} / \mathrm{mL}$. Because of its polar nature penetration of KM into cells is poor and distribution of the drug is limited to extracellular spaces. About $99 \%$ of KM is excreted unchanged in the urine. The elimination half-life of KM is approximately 2-3 hours and may be prolonged in case of renal insufficiency [105].

\section{Ethionamide}

Although ethionamide (ETH) is bactericidal in nature, large doses are required for achieving bactericidal concentrations in serum and these doses produce severe gastrointestinal disturbances. Thus, ETH could be considered as bacteriostatic agent [83]. ETH suppresses the multiplication of $M . t b$ in the concentrations ranging from 0.6 to 2.5 $\mu \mathrm{g} / \mathrm{mL}$. ETH is a prodrug structurally related to INH that when activated targets the same $\operatorname{Inh} A$ - mycolic acid biosynthesis pathway [18]. ETH is activated by a monooxygenase, $E t h A$, in the mycobacteria $[114,115]$. Mutations in the activation enzyme, EthA, and the target, InhA, result in the development of resistance to ETH [116]. ETH is reported to 
produce dose limiting gastrointestinal toxicity. Hepatotoxicity and CNS toxicity such as psychosis, peripheral neuritis and visual disturbances have also been reported [83].

Ethionamide is practically insoluble in water and is unstable on exposure to light. ETH has a low $n$-octanol-water partition coefficient $(0.705)$ and falls under BCS classIV.

After oral administration ETH is not subject to any appreciable first-pass metabolism. Peak concentrations of about $2 \mu \mathrm{g} / \mathrm{mL}$ occur 3 hours after $250 \mathrm{mg}$ of drug intake. ETH is widely distributed in the body including CSF. It undergoes extensive metabolism yielding several metabolites. ETH is eliminated quickly from the body with an elimination half-life of 2-3 hours. ETH inhibits acetylation of INH in vitro. About 1\% of the drug is excreted unchanged via the kidneys [30, 62, 90, 105].

\section{Ciprofloxacin}

The fluoroquinolones ciprofloxacin (CIP), levofloxacin, ofloxacin and sparfloxacin have been used in TB therapy and ciprofloxacin and ofloxacin are currently used as second-line agents for the treatment of MDR-TB in the USA and other countries [117]. CIP is bactericidal against M.tb. It acts by inhibiting DNA synthesis, targeting the $D N A$ gyrase $A$ and $B$ subunits. Mutations in the $G y r A$ and $G y r B$ subunits are responsible for the development of resistance to CIP [24].

CIP exists as free base and a hydrochloride salt. Aqueous solubility of the free base $(1.1 \mathrm{mg} / \mathrm{L})$ is much lower than the hydrochloride salt $(10 \mathrm{mg} / \mathrm{mL})$. CIP has a $\mathrm{pKa}$ value of 6.09, and thus exists in an ionized state in the gastrointestinal tract. The $n$ octanol-water partition coefficient $(\log \mathrm{P})$ of CIP is reported as 1.335 [54]. Based on aqueous solubility and $\log \mathrm{P}, \mathrm{CIP}$ can be classified as a BCS class-III compound, i.e., a high solubility and low permeability drug.

CIP is readily absorbed after oral administration with peaks occurring in less than 2 hours. The absolute bioavailability is approximately $70 \%$ without appreciable loss by first pass metabolism. CIP is widely distributed in the body and about $20-40 \%$ of the drug is bound to plasma proteins. CIP is metabolized primarily in the liver and four metabolites have been identified in urine accounting for about $15 \%$ of the administered dose. CIP has an elimination half-life of 3-6 hours in the body. About $40-50 \%$ of the administered dose is excreted unchanged in the urine [61].

\section{Ofloxacin}

Ofloxacin (OFL) is also a fluoroquinolone antibiotic used as a second-line agent for the treatment of MDR-TB. OFL, like CIP, is bactericidal against TB and acts by inhibiting the enzyme DNA gyrase. Resistance to OFL can also confer cross-resistance to other agents in this class of antibiotics [117]. 
OFL is more soluble in water $(28.3 \mathrm{mg} / \mathrm{mL})$ than CIP. OFL has a reported $\log P$ value of 1.268. Based on reported values of aqueous solubility and $\log \mathrm{P}$, OFL can be classified as a BCS class-III drug, i.e., a high solubility and low permeability drug similar to CIP $[51,54]$.

OFL is almost completely absorbed (85-95\%) from the gut after oral administration. Peak plasma concentrations are reached within 1-2 hours after drug intake. OFL is widely distributed in the body and about $20-30 \%$ of the drug is bound to plasma proteins. In contrast to CIP, OFL is not metabolized in the body and the majority of drug $(75 \%)$ is excreted unchanged in the urine [66-68].

\section{Cycloserine}

Cycloserine (CS) shows moderate activity against $M . t b$, with inhibitory activity at concentrations of $5-20 \mu \mathrm{g} / \mathrm{mL}$ in vitro. No cross-resistance has been observed between CS and other anti-tuberculosis agents. CS is a cell wall peptidoglycan synthesis inhibitor. It blocks the action of D-alanine racemase $(A l r)$ and D-alanine: D-alanine ligase $(D d l)$ $[118,119]$. Alr mediates L-alanine to D-alanine conversion; D-alanine then serves as a substrate for $D d l$. However, the mechanism of resistance to CS therapy is so far unclear [24].

CS shows good water solubility $(100 \mathrm{mg} / \mathrm{mL})$ and is practically insoluble in organic solvents [60]. CS is a hydrophilic drug with an $n$-octanol-water partition coefficient $(\log \mathrm{P})$ of -1.631 . Based on aqueous solubility and $\log \mathrm{P}, \mathrm{CS}$ can be classified as a BCS class-III drug, i.e., a high solubility and low permeability drug.

CS is well absorbed (70-90\%) after oral administration with peak plasma concentrations occurring within 3-4 hours of drug intake. CS is extensively distributed throughout the body including CSF. Very little of the drug undergoes metabolism and most of it is eliminated renally as unchanged drug (70\%). CS has an elimination half-life of about 10 hours. The elimination half-life may be prolonged in patients with renal insufficiency $[62,90,120]$.

\section{Thiacetazone}

Thiacetazone is bacteriostatic in nature and is considered obsolete for antitubercular pharmacotherapy [121] as it produces severe skin reactions including Steven-Johnson syndrome [83].

Thiacetazone is only slightly soluble in water. It is readily absorbed from the gut. Peak concentrations occur within 4-5 hours after drug administration. It is distributed widely into different tissues in the body with varying tissue concentrations. The drug 
undergoes rapid elimination from the body and has an elimination half-life of 4 hours. About $42 \%$ of the drug is excreted unchanged in the urine $[30,62,90,105]$.

\section{Novel Compounds/Therapies}

There has been a lot of interest in developing new compounds/therapies for the treatment of tuberculosis in view of the emergence of MDR and XDR strains of TB. Chemical structures of new and investigational anti-TB compounds are shown in Figure 1-2. In the following is a brief discussion on a novel set of compounds for which the treatment of tuberculosis has recently been approved as a new indication and compounds that are currently under clinical investigation for treatment of TB.

\section{Rifapentine}

Rifapentine, a newer agent in the class rifamycins, was approved for the treatment of pulmonary tuberculosis by the US Food and Drug Administration in 1998. Rifapentine inhibits the growth of M.tb in vitro at concentration of $0.04 \mu \mathrm{g} / \mathrm{mL}$. The mechanism of action of rifapentine is similar to that of rifampin [64].

The absolute bioavailability of rifapentine has not been determined. The relative bioavailability with an oral solution was found to be approximately $70 \%$ after a $600 \mathrm{mg}$ oral dose in healthy volunteers. Peak serum concentrations ranging from 8 to $30 \mu \mathrm{g} / \mathrm{mL}$ occur within 5-6 hours after a $600 \mathrm{mg}$ oral dose of rifapentine. A high fat meal increases bioavailability of the drug by about 50\% [64]. Rifapentine and its active metabolite, 25desacetyl rifapentine, have excellent penetration into macrophages with ratios of intracellular vs. extracellular concentration of 24-60 and 7, respectively [122]. Rifapentine is $97.7 \%$ bound to plasma proteins and has an elimination half-life of about 14-18 hours. It is hydrolyzed by intestinal and hepatic esterases to its desacetylated metabolite. In the gastrointestinal tract rifapentine also undergoes non-enzymatic, acid mediated degradation to a 3-formyl derivative. Rifapentine is a weak inducer of CYP enzymes and its induction capacity lies somewhere in-between rifampin and rifabutin. Auto-induction of metabolism has not been observed on repeated administration [64]. Following a single $600 \mathrm{mg}$ oral dose of radiolabeled rifapentine to four healthy volunteers, $17 \%$ of the total radioactivity was recovered in the urine and $70 \%$ in the feces [123]. Due to its high activity and long elimination half-life, rifapentine has compared to rifampicin the potential advantage of only needing to be administered twice weekly in the intensive phase of treatment and once weekly in the continuation phase of treatment. With this regimen, rifapentine reached similar efficacy measured as sputum culture conversion rates as rifampicin administered once daily in the intensive phase and twice weekly in the continuation phase of treatment [124]. 


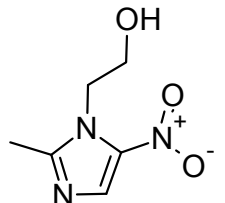

Metronidazole

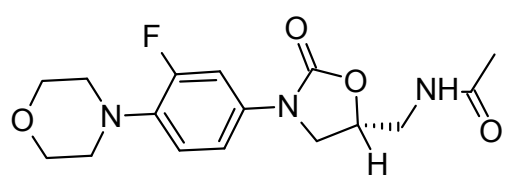

Linezolid

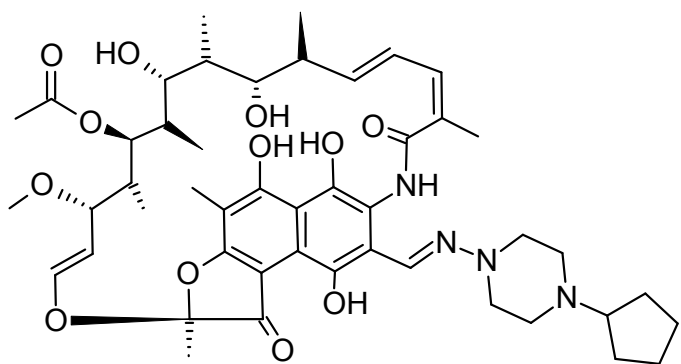

Rifapentine

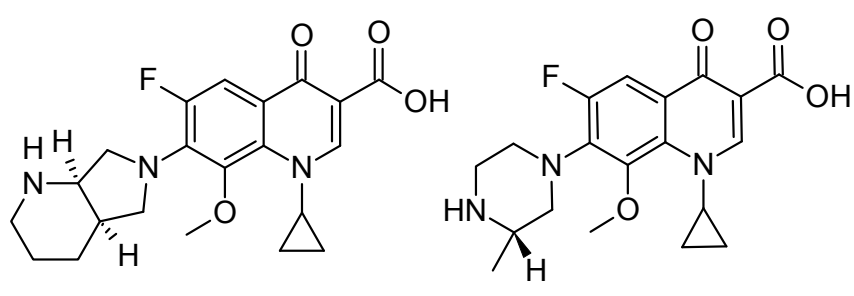

Gatifloxacin

Moxifloxacin

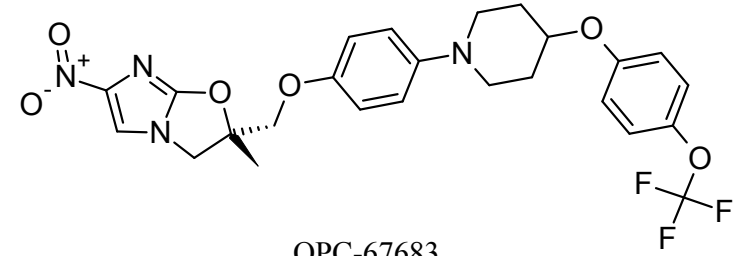

OPC-67683

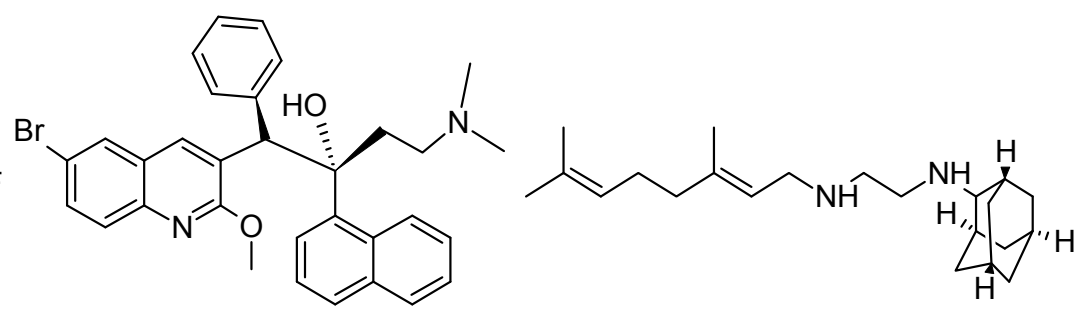

PA-824

TMC207 (R207910)

SQ109

Figure 1-2. Chemical Structures of Newer and Investigational Anti-Tuberculosis Compounds. 


\section{Moxifloxacin}

The fourth generation fluoroquinolone moxifloxacin is perhaps the most active quinolone antibiotic against M.tb [125]. Moxifloxacin is active against TB equivalent to or greater than INH at a dose level of $25 \mathrm{mg} / \mathrm{kg}$ [126]. In a mouse model, moxifloxacin monotherapy was equally effective as INH, and the combination of moxifloxacin with INH was more effective than each drug alone [127]. Moxifloxacin has also shown early bactericidal activity comparable to INH and rifampin in human subjects [128].

Moxifloxacin is readily absorbed from the gastrointestinal tract with an absolute oral bioavailability of approximately $90 \%$. Peak concentrations occur within 1-3 hours after drug administration. Co-administration with a high fat meal does not affect absorption of the drug. Moxifloxacin is approximately $50 \%$ bound to plasma proteins. It is extensively distributed throughout the body with higher tissue levels than the corresponding plasma concentrations and has a volume of distribution of 1.7-2.7 L/kg. Moxifloxacin exhibits excellent penetration into alveolar macrophages and the epithelial lining fluid with a mean tissue to plasma ratio of 21.2 and 8.7 , respectively. It is metabolized by glucuronidation ( $\sim 14 \%$ of the dose) and sulfation ( $\sim 38 \%$ of the dose). The CYP enzyme system is neither involved in the metabolism of moxifloxacin nor is it affected by moxifloxacin. The sulfate conjugate is excreted primarily in the feces whereas glucuronide conjugated is excreted in the urine. About $45 \%$ of the drug is excreted unchanged in the urine $(\sim 20 \%)$ and feces $(\sim 25 \%)$. Moxifloxacin has a mean elimination half-life of 12-14 hours [129].

\section{Gatifloxacin}

Like moxifloxacin, gatifloxacin has also shown promising activity against $M . t b$ either in combination therapy or in monotherapy in mouse models [130]. Gatifloxacin has been removed from the US market because of its adverse effects on glucose homeostasis (disturbances in blood glucose levels, hypoglycemia and hyperglycemia) in both diabetic and non-diabetic patients. Gatifloxacin exhibited potent activity against TB and no crossresistance with other classes of drugs was observed [130]. It has shown to be active against occasionally dividing bacteria in an in vitro study [131,132]. Early bactericidal activity of gatifloxacin against TB is currently being investigated in an NIH sponsored phase II clinical trial.

Gatifloxacin exhibits temperature dependent water solubility, with maximum solubility $(40-60 \mathrm{mg} / \mathrm{mL})$ occurring in a $\mathrm{pH}$ range of 2-5[70].

Gatifloxacin is well absorbed after oral administration and peak concentrations occur within 1-2 hours after drug intake. Its absolute bioavailability is $96 \%$. Food does not affect the absorption of the drug from the gastrointestinal tract. Plasma protein binding of gatifloxacin was found to be $20 \%$ in healthy volunteers. Gatifloxacin undergoes rapid tissue distribution after oral administration and achieves high concentrations in respiratory fluids and tissues such as alveolar macrophages, lung 
parenchyma, bronchial mucosa, sputum and lung epithelial lining fluid. It undergoes limited biotransformation in the body and is excreted to more than $70 \%$ unchanged in the urine. Less than $1 \%$ of the drug is recovered in the urine as metabolites. The mean elimination half-life of gatifloxacin ranges from 7-14 hours and is independent of dose and route of administration [70].

\section{Linezolid}

Linezolid is an oxazolidinone compound approved recently for gram-positive infections. It showed good activity against drug sensitive and resistant strains of M.tb both in vitro and in animal studies [133]. However, long-term use of linezolid is associated with myelosuppression and neurotoxicity. Although some recent studies have shown the efficacy of linezolid in treating MDR-TB [134, 135], severe adverse effects have been reported during its long-term use at a dose of $600 \mathrm{mg} /$ day [136]. Hence, linezolid may only be considered for treating MDR-TB.

Linezolid has an aqueous solubility of about $3 \mathrm{mg} / \mathrm{mL}$. The $n$-octanol-water partition coefficient $(\log \mathrm{P})$ of linezolid is reported as 0.232 [54]. Based on aqueous solubility and $\log \mathrm{P}$, linezolid can be classified as a BCS class-III drug.

Linezolid is rapidly and completely absorbed after oral administration with an absolute bioavailability of approximately $100 \%$. Therefore, linezolid can be administered orally or intravenously without dosage adjustment. Peak concentrations are reached within 1-2 hours after drug intake. Food does not affect the extent absorbed, however, the peak plasma concentration $\mathrm{C}_{\max }$ is decreased by $17 \%$ and the time at which the peak concentration occurs $\left(\mathrm{T}_{\max }\right)$ is delayed from 1.5 hours to 2.2 hours when high fat food is given with linezolid. Linezolid is readily distributed into highly perfused organs and is about $31 \%$ bound to plasma proteins. It is primarily metabolized by oxidation of the morpholine ring, producing two inactive metabolites. Linezolid is not detectably metabolized by enzymes of CYP system in vitro and it does not inhibit the activities of clinically significant CYP enzymes such as CYP1A2, 2C9, 2C19, 2D6, 2E1 and 3A. Under steady-state conditions, approximately $50 \%$ of the dose is excreted as metabolites and $30 \%$ as unchanged drug in the urine. Linezolid has a mean elimination half-life of about 5 hours [137].

\section{Metronidazole}

Metronidazole is a well-known anti-infective agent that has never been used for treatment of TB because it is inactive against rapidly growing aerobic TB cultures and also in mouse infection disease models of TB. Recent experiments, however, have shown it to be active against anaerobic cultures in vitro [138-140]. It has been suggested that the disease pathology in humans is significantly different to that of the mouse, with closed caseous necrotic lesions that are highly anoxic and are likely to contain anaerobic bacilli $[74,141]$, which may be susceptible to metronidazole. Studies in rabbit models of TB 
that recapitulate this feature have demonstrated metronidazole to be highly effective [142]. These studies suggest that metronidazole may have unique activity against anaerobic subpopulations of TB bacilli. Because anoxic bacteria are highly resistant to sterilizing effects of first-line drugs, such subpopulations of bacilli are thought to be responsible for extending the duration of TB chemotherapy. A clinical trial sponsored by the National Institutes of Health has started recruiting patients in South Korea to evaluate the ability of metronidazole to kill anaerobic subpopulation of M.tb within MDR-TB patients [142].

Metronidazole has an aqueous solubility of about $10 \mathrm{mg} / \mathrm{mL}$ and an $n$-octanolwater partition coefficient of -0.262 [54]. The low value of $\log \mathrm{P}$ indicates hydrophilicity of the compound. Based on these properties, metronidazole can be classified as a BCS class-III drug.

Metronidazole is well absorbed after oral administration with peaks occurring 1-2 hours after drug intake. Metronidazole exhibits linear pharmacokinetics, i.e., plasma concentrations and exposure follow dose proportionality. The drug is distributed widely throughout the body and less than $20 \%$ of the circulating metronidazole is bound to plasma proteins. In CSF, saliva and milk, metronidazole achieves concentrations similar to those found in plasma. Further, bactericidal concentrations have also been observed in the pus from hepatic abscesses. Metronidazole is primarily metabolized by side chain oxidation and glucuronide conjugation. About $20 \%$ of the drug is excreted unchanged in urine. Metronidazole has an elimination half-life of about 8 hours in healthy volunteers [143].

\section{PA-824}

PA-824 is a novel nitroimidazopyran derivative currently under development by the Global Alliance for TB Drug Development [77]. PA-824 acts mainly by inhibiting the synthesis of cell wall components through molecular targets that are yet to be identified. PA-824 showed high activity against drug sensitive and drug resistant strains of M.tb, indicating absence of cross-resistance to current TB drugs [144]. Furthermore, PA-824 exhibits activity against both replicating and non-replicating strains of bacteria in vitro making it a promising molecule for reducing the duration of therapy. PA-824 entered human phase I trials in June 2005 [145].

PA-824 is highly lipophilic and exhibits poor aqueous solubility $(\sim 20 \mu \mathrm{g} / \mathrm{mL})$ [146]. This poor solubility resulted in inefficient drug delivery in animal models. Lipid coated cyclodextrin complexation improved aqueous solubility as well as the drug's oral bioavailability from about 2 to $40 \%$ in mice. PA- 824 is widely distributed in mice and has an excellent tissue penetration in the lungs and spleen with tissue to plasma ratios of about 5 and 4, respectively [146]. Concentrations of PA-824 were not significantly different in mice when given alone or in various combination regimens including first line TB drugs such as RIF, INH and PZA. This suggests that PA-824 does not produce significant drug interactions with the first-line TB drugs. Peak serum concentrations 
achieved after a single oral dose of PA-824 in mice are 80 to 110 fold higher than the MIC of $0.25 \mu \mathrm{g} / \mathrm{mL}$ [144].

\section{OPC-67683}

OPC-67683 is a nitro-dihydroimidazooxazole derivative currently being developed by Otsuka Pharmaceuticals [147, 148]. In vitro data suggest that OPC-67683 is highly active against both drug sensitive and drug resistant subpopulations of bacteria (MIC 0.006-0.024 $\mu \mathrm{g} / \mathrm{mL}$ ). No cross-resistance has been observed with any of the existing first-line drugs. In addition, OPC-67683 exhibited very high intracellular activity against TB in a macrophage assay $[147,148]$. Phase I safety, tolerability and pharmacokinetic testing and a 7-day early bactericidal activity (EBA) study at a dose of $400 \mathrm{mg}$ have been reported to be completed. A new formulation is being tested to minimize fed vs. fasting effect in a multiple dose, multi-center extended EBA (14 days) study at four dose levels $(100,200,300$ and $400 \mathrm{mg})$. No results have been published yet [149].

In a murine experimental model of TB, OPC-67683 exhibited the longest terminal half-life $(7.6 \mathrm{hr})$ compared to all other first line drugs. Peak plasma concentration $\left(\mathrm{C}_{\max }\right.$ $0.297 \mu \mathrm{g} / \mathrm{mL})$ occurred about 6 hours after drug administration $(2.5 \mathrm{mg} / \mathrm{kg})$. The oral bioavailability of the compound is in the range of 35-60\% in mice, rats and dogs. OPC67683 is well distributed into tissues with a tissue to plasma ratio of about 3-7 in the lungs. In vitro metabolism with animal and human microsomes suggests that the drug is not metabolized by CYP enzymes and neither stimulates nor inhibits CYP enzymes at concentrations up to $100 \mu \mathrm{M}$. This suggests that clinically significant drug interactions are not expected with other CYP metabolized drugs at therapeutic levels [148].

\section{TMC207}

The diaryl quinoline TMC207 (previously, R207910) is a member of a new class of anti-mycobacterial agents currently being developed by Tibotec Pharmaceuticals, a research subsidiary of Johnson \& Johnson. It has been found to have a new mode of action that is different from the existing anti-TB agents, implying a low probability of cross-resistance. TMC207 inhibits the proton pump of the M.tb ATP synthase, the main source of energy for mycobacteria [43]. It has been recently identified that the oligomeric subunit c (AtpE) of ATP synthase is the target of TMC207 [150]. A remarkable feature of TMC207 is its exceptional specificity for mycobacteria. Though ATP synthase is a ubiquitous enzyme found in most living organisms, a very limited sequence similarity between the mycobacterial and human AtpE proteins was observed [151]. The compound showed potent early bactericidal activity matching or exceeding that of INH in a nonestablished murine mouse model [171]. It also showed synergistic effects when given with pyrazinamide in murine models $[153,154]$. Phase IIa proof-of-concept studies were completed recently in TB patients at three dose levels, 25, 100 and $400 \mathrm{mg}$ given once daily for one week. Bactericidal activity was observed at a dose of $400 \mathrm{mg}$. The 
compound was well tolerated and no major safety concerns were identified. Dosing regimen and treatment duration needed for sterilization has not been defined in humans yet. Phase II trials to determine dosing regimen, long-term safety and efficacy are planned to start soon [155].

In a single ascending-dose pharmacokinetic study, TMC207 was well tolerated by human volunteers without any severe adverse effects. It is well absorbed after oral administration and peak concentrations $\left(\mathrm{C}_{\max }\right)$ occur about $5 \mathrm{hr}$ after the dose. Absorption of TMC207 is increased twofold with food. Plasma drug concentrations decline triexponentially after $\mathrm{C}_{\max }$ is reached. The compound exhibits linear pharmacokinetics in healthy volunteers as well as TB patients, i.e., a proportional increase in $\mathrm{C}_{\max }$ and area under the curve (AUC) in the dose range tested (25-700 mg). A multiple-ascending dose study suggests an 'effective half-life' of 24 hours. Steady state blood levels were not achieved until 14 days of therapy due to the long terminal half-life. Steady state plasma concentrations were higher than those effective in a mouse model of TB [43]. TMC207 is metabolized by CYP3A4 enzymes. Thus, enzyme induction during co-administration with rifampin lowered TMC207 levels by 50\%.

TMC207 is an attractive candidate molecule for TB therapy because of its high potency against drug sensitive and drug resistant TB strains, its novel mode of action and its long elimination half-life [151]. However, it has several potential problems for development as a first-line drug: influence of food on the oral absorption, druginteraction potential with the important first-line TB drug rifampicin, and minimal early bactericidal activity (EBA) for the first four days of therapy when compared to INH and RIF. However, EBA from days 5 to 7 is similar to INH and RIF at a dose of $400 \mathrm{mg}$. The influence of food on the oral bioavailability would likely complicate designing a standardized dosing regimen suitable for patients around the globe. Since TMC207 is metabolized by CYP3A4, it is also likely subject to drug interactions with anti-HIV therapeutics such as protease inhibitors raising concerns about its usefulness in HIV positive TB patients [156]. Tibotec is considering the evaluation of the activity of TMC207 in a phase II trial in MDR-TB patients because of the absence of RIF in the standard MDR-TB regimen and the relatively low efficacy of the existing regimen in these patients [157].

\section{SQ109}

SQ109 is a novel ethambutol analog developed from high throughput combinatorial screening [158]. SQ109 shows good activity against TB in in vitro assays including a macrophage assay and in an in vivo mouse model. It has shown efficacy and potency in inhibiting intracellular mycobacteria that is similar to that of INH and superior to ethambutol. SQ109 showed a dose dependent reduction of mycobacterial load in lungs and spleen after 28 days of therapy at $0.1-25 \mathrm{mg} / \mathrm{kg} /$ day that was equivalent to ethambutol at $100 \mathrm{mg} / \mathrm{kg} /$ day and was less potent than INH at $25 \mathrm{mg} / \mathrm{kg} /$ day [159]. On substitution for ethambutol, SQ109 showed improved efficacy in combination therapy with first-line TB drugs, isoniazid and rifampicin, in a chronic infection mouse models 
[160]. SQ109 is currently being tested for safety, tolerability and pharmacokinetics in phase I clinical trials.

SQ109 undergoes rapid but incomplete absorption following oral administration in mice, rats and Beagle dogs with peak concentrations occurring in less than an hour. The compound has an oral bioavailability of $2.4-5,12$ and $3.8 \%$ in dogs, rats and mice, respectively. After the oral administration of ${ }^{14} \mathrm{C}$ labeled compound to rats, the highest level of radioactivity was found in the liver followed by the lungs, the spleen and the kidneys. $22.2 \%$ and $5.6 \%$ of the radioactivity was found in feces and urine, respectively. The plasma protein binding of SQ109 varied from 6 to $23 \%$ depending on the species (human, rat, mouse and dog), and the compound showed a terminal half-life of 5.3, 7.4 and 29.3 hours in mice, rats and dogs, respectively [161]. In an in vitro metabolic study using 10 minute incubation with mouse, rat, dog and human microsomes, about 23, 48, 51 and $58 \%$ of SQ109 remained unchanged, respectively. The majority of SQ109 is metabolized via oxidation, epoxidation and N-dealkylation in human liver microsomes. CYP reaction phenotyping coupled with CYP specific inhibitors identified CYP2D6 and CYP2C19 to be the predominant enzymes involved in the metabolism of SQ109.

The novel antitubercular compounds PA-824, OPC-67683, TMC207 and SQ109 are all in various phases of clinical development, raising the hopes for a more effective eradication of TB. A closer look at the available data (mostly animal and few human data) and the compounds' predicted biopharmaceutic characteristics (Table 1-4) suggests that all of these compounds suffer from poor aqueous solubility. Poor solubility coupled with the high lipophilicity (characteristics of BCS class-II drugs) of these compounds could be obstacles in developing oral formulations for efficient delivery. For instance, the formulation used in the phase I and proof-of-concept studies of OPC-67683 needed to be replaced with a newer formulation [149] and PA-824 exhibited poor bioavailability in mice before a new formulation approach (lipid coated cyclodextrins) was used [146]. Furthermore, poor aqueous solubility frequently results in high interindividual variability in oral absorption, and low oral bioavailability. Food influences the absorption of BCS class-II drugs by either increasing the gastric residence time or increasing the solubility (fatty food). Influence of food on the absorption is undesirable for a TB therapeutic since it complicates the design of an effective, standardized dosing regimen suitable for TB patients worldwide. Thus, it is important to focus on the aqueous solubility and lipophilicity of novel compounds in the early stages of drug discovery to avoid potential problems in their delivery.

\section{PK/PD Parameters of Anti-TB Drugs}

Anti-infective agents, in general, have been characterized by two different patterns of killing, time-dependent and concentration-dependent killing. Intracellularly acting drugs such as aminoglycosides, fluoroquinolones and rifamycins exhibit concentration-dependent killing patterns whereas antibiotics that act on cell wall targets, such as penicillins and cephalosporins exhibit time-dependent killing patterns. PK/PD parameters such as $\mathrm{AUC} / \mathrm{MIC}$ and $\mathrm{C}_{\max } / \mathrm{MIC}$ explain the bactericidal activity of 
Table 1-4. Biopharmaceutic and Pharmacokinetic Properties of Investigational Anti-Tuberculosis Drugs.

\begin{tabular}{lccccccc}
\hline Compound & MW & $\begin{array}{c}\text { MIC } \\
(\boldsymbol{\mu g} / \mathbf{m L})\end{array}$ & $\mathbf{c l o g P}$ & $\begin{array}{c}\text { Solubility } \\
(\boldsymbol{\mu g} / \mathbf{m L})^{*}\end{array}$ & $\begin{array}{c}\text { Bioavailability } \\
(\mathbf{\%})\end{array}$ & $\begin{array}{c}\text { Half-life } \\
(\mathbf{h r})\end{array}$ & References \\
\hline PA-824 & 359.3 & $0.015-0.25$ & 1.91 & 21.6 & $2-40^{\mathrm{a}}$ & $12.8-18.3^{\mathrm{a}}$ & {$[144,162-164]$} \\
OPC-67683 & 534.5 & $0.006-0.024$ & 5.23 & 0.04 & $35-60^{\mathrm{b}}$ & $7.6^{\mathrm{a}}$ & {$[148,149,162$,} \\
TMC207 & 555.5 & $0.003-0.120$ & 6.41 & 0.08 & na & $24^{\mathrm{c}}$ & {$[43,162,163]$} \\
SQ109 & 330.6 & $0.16-0.64$ & 5.84 & 4.07 & $2.4-12^{\mathrm{b}}$ & $5.3-29.3^{\mathrm{b}}$ & {$[161-163]$} \\
\hline
\end{tabular}

*: In silico predicted solubility.

a: Data from PK studies in mice.

b: Data from PK studies in mice, rats, and dogs.

c: Data from PK studies in humans. 
concentration-dependent killers while time during which serum levels remain above MIC ( $>$ MIC) explains the efficacy of time dependent killers [165]. The magnitude of these parameters varies among organisms for reliable prediction of bactericidal effect. For fluoroquinolones, the bactericidal effect against gram-negative bacilli such as Pseudomonas aeruginosa is observed at a $\mathrm{C}_{\max } / \mathrm{MIC}$ ratio of $>12$ or AUC/MIC ratio $\geq 100-125$, with poor activity at $\mathrm{C}_{\max } / \mathrm{MIC}$ ratios $<4$. Maximum activity is achieved when the AUC/MIC ratio exceeds 250 [166]. If this holds true for mycobacterial infections, the effectiveness of rifampicin, ciprofloxacin, ofloxacin, streptomycin, amikacin and kanamycin should improve by giving larger doses less frequently. However, limited information exists on the PK/PD correlates of bactericidal activity of antitubercular agents. Important PK/PD parameters of antitubercular agents are given in Table 1-5. So far, very little or no correlation was observed between PK/PD measures and bactericidal or sterilizing activity of anti-TB agents. Correction for protein binding did not improve this correlation. PAS, a bacteriostatic agent, has surprisingly larger $\mathrm{C}_{\max } / \mathrm{MIC}$ and AUC/MIC ratios compared with rifampin and isoniazid. Recent studies in the aerosol infected mouse model have shown that RIF and INH exhibit a concentration-dependent killing pattern that correlates best with the AUC/MIC ratio [167, 168]. The same authors have also shown AUC/MIC to be a reliable predictor of efficacy for the fluoroquinolones moxifloxacin, sparfloxacin, ciprofloxacin and ofloxacin [169]. However, the PK/PD parameters identified using a mouse model can predict only the early bactericidal activity because of inherent drawbacks of these models. An important difference exists between the aerosol infected mouse model of TB and human disease. Unlike humans, the granulomas in mouse models are not progressed to caseation and liquefaction. Further, after the onset of the chronic phase of the disease, unlike humans, the lungs and spleen of mice contain high numbers of persisting bacteria [76]. Non-human primate models of TB that recapitulate the human disease are ideal for identifying the clinically relevant PK/PD predictors of sterilization efficacy of anti-TB agents. However, these models are very expensive and are not suitable for screening compounds in the early stages of the drug development process. Thus, more studies are needed to fill this gap between drug discovery and clinical testing and provide definitive guidance in antitubercular drug development.

In spite of remarkable developments in the fields of mycobacteriology and drug development, the number of antitubercular drug molecules that have recently reached clinical trials is small. The major bottlenecks in antitubercular drug development include lack of inexpensive animal models to predict the efficacy of compounds under development, a poor understanding of predictive PK/PD measures and a poor understanding of latent TB infection. Recent reports on the emergence of extensively drug-resistant tuberculosis (XDR-TB) [3] are concerning and reinforce the need for a speedy development of agents with novel modes of action and lack of cross-resistance. Novel molecules such as OPC-67683, TMC207 and PA-824 are promising candidates to treat drug resistant TB and are expected to reach the market by 2012 [171]. TB pharmacotherapy, however, also needs to be improved by developing more efficient dosing regimens for the existing anti-TB drugs that allow for shortening the duration and improving the adherence to therapy. 
Table 1-5. PK/PD Parameters of Anti-Tuberculosis Drugs.

\begin{tabular}{|c|c|c|c|c|c|c|c|c|c|c|c|}
\hline Target & Drug & $\mathrm{C}_{\max } / \mathrm{MIC}$ & $\mathbf{T}>\mathbf{M I C}$ & AUC/MIC & $\begin{array}{l}\text { Protein } \\
\text { Binding }\end{array}$ & fu & $\begin{array}{c}f u^{*} \\
C_{\max } / M I C\end{array}$ & $\begin{array}{c}\mathbf{f u}^{*} \\
\text { AUC/MIC }\end{array}$ & $\begin{array}{c}\text { Bactericidal } \\
\text { Activity }\end{array}$ & $\begin{array}{c}\text { Sterilizing } \\
\text { Activity }\end{array}$ & References \\
\hline \multirow{7}{*}{$\begin{array}{l}\text { Cell wall } \\
\text { Components }\end{array}$} & Isoniazid (Slow) & \multirow{2}{*}{40} & 18 & 19.2 & \multirow{2}{*}{ Negligible } & \multirow[t]{2}{*}{0.99} & \multirow{2}{*}{39.6} & 19.01 & \multirow{2}{*}{+++} & \multirow{2}{*}{+} & \multirow{2}{*}[31,62,83]{} \\
\hline & Isoniazid (Fast) & & 9 & 11.6 & & & & 11.48 & & & \\
\hline & Ethambutol & 10 & 13 & 23.4 & $6-30 \%$ & 0.7 & 7 & 16.38 & ++ & + & {$[31,62,83]$} \\
\hline & Pyrazinamide & 3.8 & na & 52 & $5-10 \%$ & 0.9 & 3.42 & 46.8 & + & +++ & {$[31,62,170]$} \\
\hline & Ethionamide & 1.6 & 1.5 & 1 & $30 \%$ & 0.7 & 1.12 & 0.7 & ++ & + & {$[31,62,83]$} \\
\hline & Thiacetazone & 1.3 & 5.5 & 1.2 & $95 \%$ & 0.05 & 0.065 & 0.06 & + & + & {$[31,62,83]$} \\
\hline & Cycloserine & 3.8 & 22.5 & 195.5 & na & na & na & na & + & + & {$[31,62,83]$} \\
\hline \multirow[t]{5}{*}{$\begin{array}{l}\text { Intracellular } \\
\text { Targets }\end{array}$} & Rifampicin & 24 & 9 & 39.9 & $60-90 \%$ & 0.1 & 2.4 & 3.99 & +++ & +++ & {$[31,62,83]$} \\
\hline & Streptomycin & 10 & 8 & 124.5 & $35-57 \%$ & 0.43 & 4.3 & 53.54 & + & + & {$[31,62,83]$} \\
\hline & PAS & 75 & 4 & 153.7 & $15 \%$ & 0.85 & 63.75 & 130.6 & + & + & {$[31,62,83]$} \\
\hline & Ciprofloxacin & 5 & 10.5 & 16.9 & $20-40 \%$ & 0.6 & 3 & 10.14 & + & + & {$[31,62,83]$} \\
\hline & Ofloxacin & 5 & 15.5 & 47.4 & $20-30 \%$ & 0.7 & 3.5 & 33.18 & + & + & {$[31,62,83]$} \\
\hline
\end{tabular}

$\mathrm{C}_{\max } / \mathrm{MIC}$ : Ratio of peak serum concentration to minimum inhibitory concentration.

$\mathrm{T}>\mathrm{MIC}: \%$ of time during which the serum concentration remains above the MIC.

AUC/MIC: Ratio of area under plasma concentration-time curve to MIC.

Bactericidal Activity: Killing of rapidly dividing bacteria.

Sterilizing Activity: Total eradication of bacteria that includes rapidly dividing and dormant subpopulations.

fu: Fraction of unbound drug.

fu $\mathrm{x}_{\mathrm{max}} / \mathrm{MIC}$ and fu $\mathrm{x}$ AUC/MIC: $\mathrm{PK} / \mathrm{PD}$ parameters calculated using free fraction of the drug. 


\section{Central Hypothesis}

The effectiveness of an antibacterial agent depends upon its ability to reach the site of infection (intracellular and/or interstitial) in a sufficiently high unbound concentration and to stay at the site of infection for a sufficiently long period of time. The aspect of presentation of drugs to bacteria is governed by the pharmacokinetics (PK) of the drug. The mere presence of an antibacterial agent at the site of infection is not sufficient for its activity; the compound also needs to have the ability to penetrate the bacterial cell wall to achieve sufficient intracellular concentrations and the compound should have affinity for its target to achieve antimicrobial effect (intrinsic activity). The aspects of a drug's antimicrobial activity are termed pharmacodynamics (PD) [172]. It is thus readily apparent that the in vivo activity of an anti-infective agent is determined by its intrinsic antimicrobial activity and free, unbound concentration in the target tissue, since only free, non-protein bound drug is pharmacologically active.

Traditionally, the antimicrobial drug development process has been a trial and error approach in which different candidate compounds were tested for in vitro and subsequently in vivo antimicrobial activity. This process is inefficient, resource intensive and time consuming as it requires a large number of compounds to be screened to identify a potential lead for further development. Pharmacokinetic/pharmacodynamic (PK/PD) models in an integrated fashion provide powerful predictive capabilities for drug efficacy in vivo $[173,174]$, and allow in silico modeling and simulation of the effectiveness of different dosing regimens and treatment modalities $[52,175]$. The utilization of PK/PD principles from the early stages of drug development is believed to result in scientifically driven, evidence-based, more focused and accelerated drug development process [176-179]. The utilization of PK/PD principles in designing dosing regimens and guiding drug development processes has been the common practice for various classes of antibiotics in non-mycobacterial infections, especially for fast-growing microorganisms [165, 180-186]. However, so far very limited information exists on the $\mathrm{PK} / \mathrm{PD}$ relationships of existing or novel anti-tubercular drugs and on the utility of these relationships in guiding a drug development process. We hypothesized that an iterative $\mathrm{PK} / \mathrm{PD}$ guided paradigm involving pharmacokinetically guided lead optimization followed by a pharmacodynamic evaluation in in vitro pharmacokinetic/pharmacodynamic model would facilitate the identification of antimycobacterial lead compounds suitable for further development. We investigated this hypothesis for a novel class of nitrofuranylamides targeted against $M$. tuberculosis.

In specific aim 1 (Chapter 2), we developed an iterative pharmacokinetically guided lead optimization paradigm based on a set of biopharmaceutic and in vivo pharmacokinetic properties. We applied this approach in the lead optimization of a novel class of nitrofuranylamides with antimycobacterial activity.

In specific aim 2 (Chapter 3), we developed a novel in vitro PK/PD model for characterizing the time course of bacterial kill rates for antibiotic dosing regimens against slow growing microorganisms, thereby capturing the dynamic interplay between microorganisms and changing drug concentrations as encountered during prolonged 
antibiotic pharmacotherapy in vivo. We validated the in vitro $\mathrm{PK} / \mathrm{PD}$ model by characterizing the time course of bacterial kill rates for dosing regimens of a first line anti-tuberculosis drug, isoniazid (INH) using M. bovis BCG as a model organism.

In specific aim 3 (Chapter 4), we determined time course of bacterial kill rates for dosing regimens of a nitrofuranylamide lead compound, Lee 1106 in the in vitro PK/PD model. Based on these data, we developed an in silico pharmacodynamic model for describing the Lee 1106 mediated bacterial kill. We applied this in silico pharmacodynamic model to predict the bactericidal effect of Lee 1106 at different, untested dosing regimens by numerical simulation experiments, thereby gaining insights on the potential in vivo antimycobacterial activity of Lee 1106. 


\section{CHAPTER 2. PHARMACOKINETICALLY-GUIDED LEAD OPTIMIZATION OF NITROFURANYLAMIDE ANTI-TUBERCULOSIS AGENTS*}

\section{Introduction}

Mycobacterium tuberculosis (M.tb) is the causative agent of TB in humans. As indicated by World Health Organization statistics, one-third of the world population is currently infected with TB, and it is anticipated that close to $10 \%$ of these infected individuals develop active TB at some point in their lifetime. TB is the second leading cause of infectious disease mortality in the world with approximately 2 to 3 million deaths per year [14]. Association of TB with HIV has proven to be fatal; a quarter of a million TB deaths are HIV infection associated [1]. Current pharmacotherapeutic regimens require treatment of TB with multiple drugs, including isoniazid, rifampicin, ethambutol and pyrazinamide for a period of six months. Treatment of drug resistant TB is more difficult requiring the usage of second-line agents that are associated with increased toxicity [187]. Adherence to prolonged treatment regimens required to kill persistent or latent bacteria and thus clear the infection is often poor, leading to treatment failures and development of resistance to therapy. Recently, the emergence of XDR-TB [3] has raised concerns that we may lose control over the spread of TB. Though currently there are intensive efforts to develop new TB therapies, it is worth reflecting that no new major classes of drugs have been approved for the treatment of TB since the introduction of the rifamycins into the market in 1971 [188]. Thus, the availability of more potent antibiotics that could effectively reduce the duration of therapy against $M . t b$ is highly desirable.

Besides good in vivo activity, an ideal TB drug should have minimal side effects, high oral bioavailability, and a favorable pharmacokinetic profile, i.e., linear pharmacokinetics and a long terminal half-life that would allow once-a-day dosing with predictable and reproducible systemic exposure, and compatibility with existing HIV medications for joint therapy. In an effort to develop new and more potent therapies to treat TB, a novel class of anti-infectives with high in vitro activity against M.tb, nitrofuranylamides, has recently been characterized by our group [189-192].

The main objective of the current work is to gain an understanding of the preclinical pharmacokinetics of the nitrofuranylamide series of compounds, and to improve the pharmacokinetic properties of these compounds via structural modification. Because optimization of pharmacokinetic properties is frequently a rate limiting step in the preclinical development of anti-tuberculosis agents [12, 193, 194], the pharmacokinetically-guided lead optimization used in this publication may also be useful for screening other compounds in early drug discovery projects.

*This chapter adapted with permission. Budha, N.R., et al., Pharmacokinetically-guided lead optimization of nitrofuranylamide anti-tuberculosis agents. AAPS J, 2008. 10(1): p. 157-65 [235]. 


\section{Materials and Methods}

\section{Chemicals and Reagents}

All tested nitrofuranylamides were synthesized in the laboratory of Dr. R.E. Lee at the University of Tennessee Health Science Center, Memphis, TN, including Lee 562 (5-Nitro-furan-2-carboxylic acid [6-(4-benzyl-piperazin-1-yl)-pyridin-3-ylmethyl]amide), Lee 878 (1-benzyl-4-\{4-[(5R)-3-(5-nitro-2-furyl)-4, 5-dihydroisoxazol-5-yl] phenyl $\}$ piperazine), Lee 952: $N$-methyl- $N$-[4-(2-morpholin-4-ylethoxy) benzyl]-5-nitro2-furamide, and Lee 1106 (1-\{4-[3-(5-nitro-2-furyl)-4, 5-dihydroisoxazol-5-yl] phenyl $\}$ 4-[4-(trifluoromethoxy) phenoxy] piperidine) (Figure 2-1). Details on the synthesis of the test compounds are described elsewhere [191, 192, 195]. Acetonitrile, HPLC grade water, acetic acid and ammonium acetate were purchased from Fisher Scientific (Pittsburgh, PA). Drug free rat plasma was purchased from Aleken Biologicals (Nash, TX).

\section{Pharmacokinetic Studies in Rats}

Catheterized male Sprague-Dawley rats (jugular vein alone for oral study and jugular vein and femoral vein for intravenous study) from Harlan Bioscience (Indianapolis, IN), weighing approximately $250 \mathrm{~g}$, were kept on a $12 \mathrm{hr}$ light/dark cycle with food and water available ad libitum. Groups of rats $(n=4-5)$ received either an intravenous (IV) or oral dose of a test compound at a dose level of $10 \mathrm{mg} / \mathrm{kg}$ or 100 $\mathrm{mg} / \mathrm{kg}$, respectively. For oral administrations, the animals were fasted overnight and until $4 \mathrm{hr}$ after administration of test compound. Serial blood samples $(\sim 250 \mu \mathrm{L})$ were collected predose and at predetermined time points postdose. Plasma was separated immediately by centrifugation $\left(3000 \mathrm{~g}\right.$ for $10 \mathrm{~min}$ at $\left.4^{\circ} \mathrm{C}\right)$ and stored at $-80^{\circ} \mathrm{C}$ until analysis. Feces and urine specimens were collected for Lee 562 and Lee 1106 for a period of 24 hours after administration. The study protocol was approved by the Institutional Animal Care and Use Committee of the University of Tennessee Health Science Center.

\section{Microsomal Incubations}

Microsomal metabolic stability of the compounds was assessed in pooled rat liver microsomal preparations (Cellzdirect, Austin, TX) by monitoring disappearance of the parent compound over an incubation period of 90 minutes. The percentage of intact parent compound in the samples was estimated by comparing analyte concentrations before and after incubation using a liquid chromatography-tandem mass spectrometry (LC-MS/MS) assay. Reactions were started by adding $25 \mu \mathrm{L}$ of microsomal protein solution $(10 \mathrm{mg} / \mathrm{mL})$ to $25 \mu \mathrm{L}$ of test compound $(50 \mu \mathrm{M})$ and $200 \mu \mathrm{L}$ of NADPH regenerating solution (1.3 mM NADP ${ }^{+}, 3.3 \mathrm{mM}$ glucose-6-phosphate, $3.3 \mathrm{mM} \mathrm{MgCl} 2$ and 1 unit/mL glucose-6-phosphate dehydrogenase in $\mathrm{pH} 7.4$ phosphate buffer solution). The mixture was incubated at $37^{\circ} \mathrm{C}$ and samples were taken at $0,15,30,45,60$ and 90 


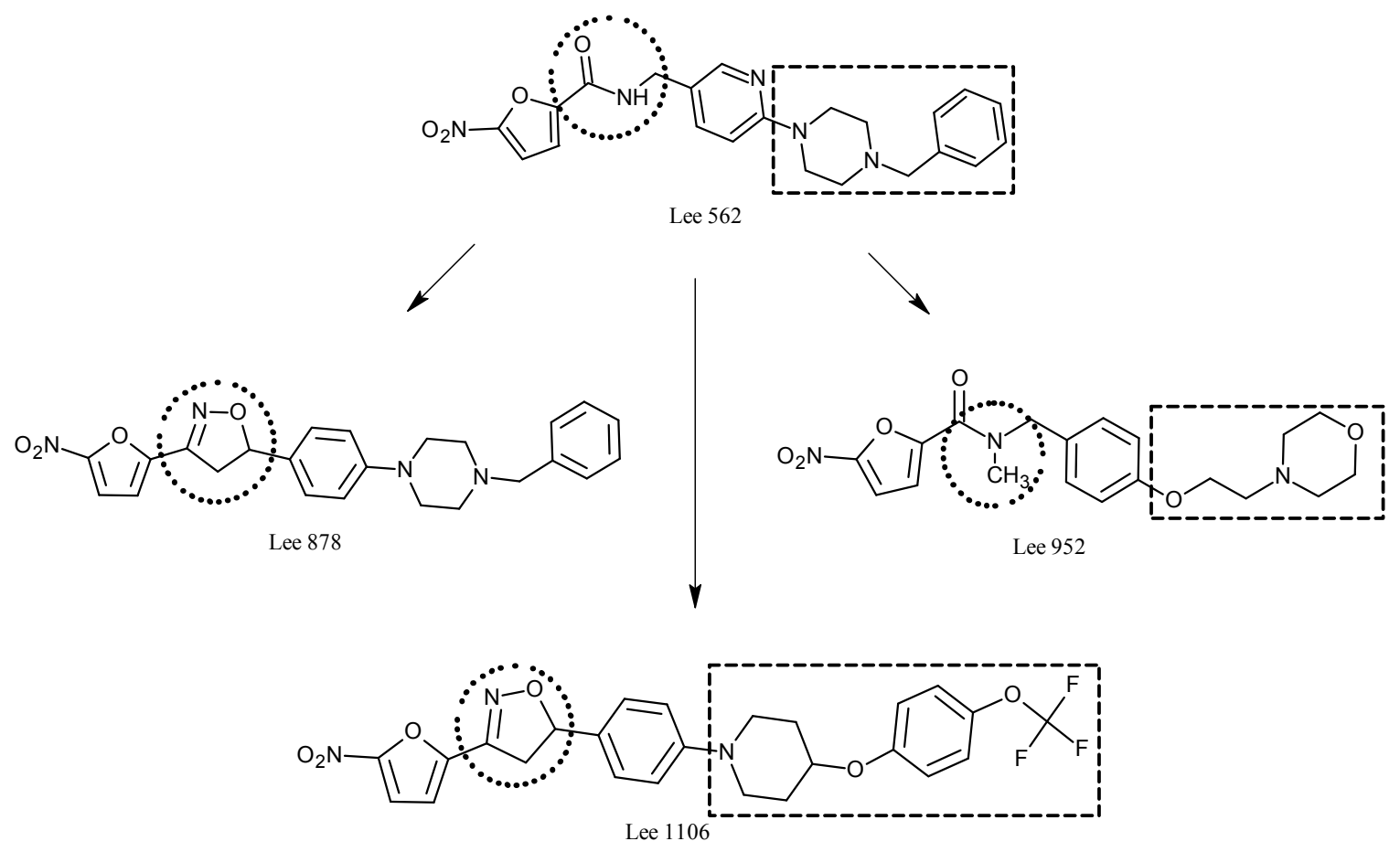

Figure 2-1. Chemical Structures of Lee 562, Lee 878, Lee 952, and Lee 1106. The boxes and circles depict important structural differences between the first generation compound Lee 562, the second generation compounds Lee 878 and Lee 952, and the third generation compound Lee 1106. 
minutes. The reaction was terminated by the addition of three volumes of ice-cold acetonitrile containing an internal standard, Lee 563. Controls were treated in a similar manner using heat-denatured microsomes. Samples were centrifuged at $3,000 \times g$ for 10 minutes at $4^{\circ} \mathrm{C}$ and the supernatants were removed for analysis. An aliquot $(10 \mu \mathrm{L})$ was analyzed by LC-MS/MS.

\section{Solubility and Lipophilicity}

Aqueous solubility of the compounds was estimated at two different $\mathrm{pH}$ values using a miniaturized shake-flask method [196]. Approximately $1 \mathrm{mg}$ of the test compound was shaken with $500 \mu \mathrm{L}$ of either $\mathrm{pH} 6.0$ or $\mathrm{pH} 7.4$ buffer in a glass vial at $400 \mathrm{rpm}$ and room temperature for 24 hours. The resulting mixture was centrifuged and the concentration in the supernatant was determined by LC-MS/MS. The octanol-water partition coefficient ( $\log \mathrm{P}$ ) of the compounds as measure of their lipophilicity was calculated using ChemDraw Ultra version 7.0 (CambridgeSoft Corporation, Cambridge, MA).

\section{Protein Binding}

Protein binding of the compounds was determined using equilibrium dialysis. Biologically relevant concentrations of test compound were prepared (low and high) in rat plasma. $200 \mu \mathrm{L}$ of the plasma sample was placed in the central chamber and $350 \mu \mathrm{L}$ of blank isotonic phosphate buffer, $\mathrm{pH} 7.4$ in the peripheral chamber of a dialysis device (MW cutoff 6000-8000, RED $\circledast$ device, Pierce Biotechnology Inc, Rockford, IL). The chambers were covered with a seal and incubated at $37^{\circ} \mathrm{C}$ for $4-6$ hours at a rocking speed of $100 \mathrm{rpm}$. At the end of incubation, the volumes of plasma and recipient buffer were measured to identify and account for volume shift, if any. Aliquots of plasma and buffer were used to determine the drug concentration using an LC-MS/MS assay. The free fraction of the drug was calculated as ratio of the concentrations in the buffer and in plasma.

\section{Plasma Concentration Measurements by LC-MS/MS}

A calibration curve ranging from 1-500 $\mu \mathrm{g} / \mathrm{L}$ was constructed for each test compound by spiking the test compound into $50 \mu \mathrm{L}$ of blank rat plasma. A structural analogue of the test compounds, Lee 563 (ethyl 4-(5-\{[(5-nitro-2-furoyl) amino] methyl $\}$ pyridin-2-yl) piperazine-1-carboxylate), was added as internal standard to all calibration standards and all plasma specimens. Plasma proteins were precipitated by the addition of three volumes of acetonitrile. The samples were centrifuged at $3000 \times \mathrm{g}$ for 10 minutes at $4^{\circ} \mathrm{C}$ and the supernatants were removed for analysis. Chromatographic separations were carried out using a Shimadzu liquid chromatograph (Shimadzu Corporation, USA) consisting of two pumps, online degasser, system controller and a CTC Leap auto sampler (Leap Technologies, Carrboro, NC). A gradient of acetonitrile and $10 \mathrm{mM}$ 
ammonium acetate was used at a flow rate of $0.3 \mathrm{~mL} / \mathrm{min}$. A Phenomenex ${ }^{\circledR}$ Luna, $3 \mu$ C18 (2), $50 \times 2 \mathrm{~mm}$ column (Phenomenex, Torrance, CA) protected with a guard column was used for the separation. $10 \mu \mathrm{L}$ of sample was injected onto the column and the eluate was led directly into an API 3000 triple-quadrupole mass spectrometer (Applied Biosystems ABI/MDS-Sciex, Foster City, CA) equipped with an electrospray ion source. The quadrupoles were operated in the positive ion mode. The resulting multiple reaction monitoring chromatograms were used for quantification using Analyst software version 1.4.1 (Applied Biosystems ABI/MDS-Sciex, Foster City, CA).

\section{Pharmacokinetic Data Analysis}

Plasma concentration-time data were analyzed by non-compartmental analysis using WinNonlin 5.0.1 (Pharsight Corporation, Mountain View, CA). The area under the plasma concentration-time curve from time 0 to infinity $\left(\mathrm{AUC}_{\text {inf }}\right)$ was calculated by the trapezoidal rule with extrapolation to time infinity. The terminal half-life $(t 1 / 2)$ was calculated as $0.693 / \lambda_{z}$, where $\lambda_{z}$ is the terminal phase rate constant. The plasma clearance (CL) was calculated using the equation $\mathrm{CL}=$ Dose $_{\text {iv }} / \mathrm{AUC}_{\text {inf, iv }}$, where Dose $\mathrm{iv}_{\text {iv }}$ and $\mathrm{AUC}_{\text {inf, iv }}$ are the IV dose and corresponding area under the plasma concentration-time curve from time 0 to infinity, respectively. Volume of distribution based on terminal phase $\left(\mathrm{V}_{\mathrm{z}}\right)$ was calculated using $\mathrm{V}_{\mathrm{z}}=$ Dose $_{\text {iv }} /\left(\lambda_{\mathrm{z}}{ }^{*} \mathrm{AUC} \mathrm{C}_{\text {inf, iv }}\right)$. The peak plasma concentration $\left(\mathrm{C}_{\max }\right)$ and the time when it occurred $\left(\mathrm{t}_{\max }\right)$ in the oral dose group were obtained by visual inspection of the plasma concentration-time curves. Clearance after oral dose $(\mathrm{CL} / \mathrm{F})$ was calculated as Dose $_{\text {oral }} / \mathrm{AUC}_{\text {inf, oral }}$. Volume of distribution after oral dose $\left(\mathrm{V}_{\mathrm{z}} / \mathrm{F}\right)$ was calculated as Dose $_{\text {oral }} /\left(\lambda_{z}{ }^{*} \mathrm{AUC}_{\text {inf, oral }}\right)$. Oral bioavailability $(\mathrm{F})$ was calculated using $\mathrm{F}=\left(\mathrm{AUC}_{\text {inf, oral }}{ }^{*}\right.$ Dose $\left._{\text {iv }}\right) /\left(\mathrm{AUC}_{\text {inf, iv }} *\right.$ Dose $\left._{\text {oral }}\right)$, where Dose oral, Dose $_{\text {iv }}, \mathrm{AUC}_{\text {inf, iv }}$, and $\mathrm{AUC}_{\text {inf, oral }}$ are the oral and IV dose and the corresponding areas under the plasma concentration-time curves from time 0 to infinity, respectively.

\section{Results}

\section{Pharmacokinetics of Lee 562}

The first evaluated lead compound was Lee 562 (Figure 2-1). The plasma concentration-time profiles of Lee 562 after single IV and oral administration are shown in panels A and B of Figure 2-2, the derived pharmacokinetic parameters in Table 2-1. Lee 562 appeared rapidly in plasma after oral administration with peak plasma levels $\left(\mathrm{C}_{\max }\right)$ observed in less than $15 \mathrm{~min}$ after administration. There was a rapid decline in the plasma levels followed by a smaller second peak about $6 \mathrm{hr}$ after the dose administration. The second peak in the profile after oral administration indicates the possibility of enterohepatic recirculation of the compound or erratic absorption. The compound was distributed extensively in the body with a volume of distribution of $28.9 \mathrm{~L} / \mathrm{kg}$. A high clearance of $12.9 \mathrm{~L} / \mathrm{hr} / \mathrm{kg}$ indicates rapid elimination. The amount of compound excreted unchanged into urine or feces was less than $0.1 \%$ of the dose by both routes of 
A)

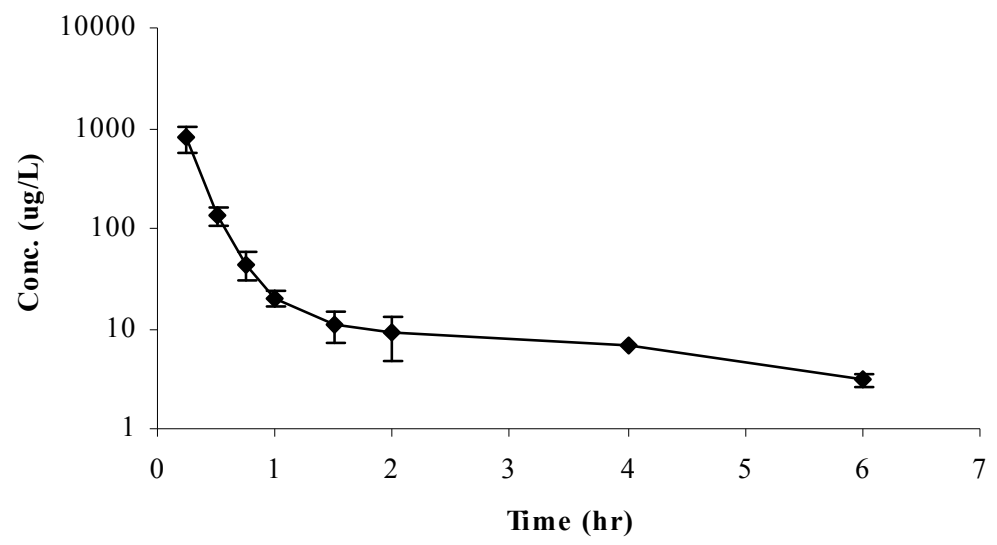

B)

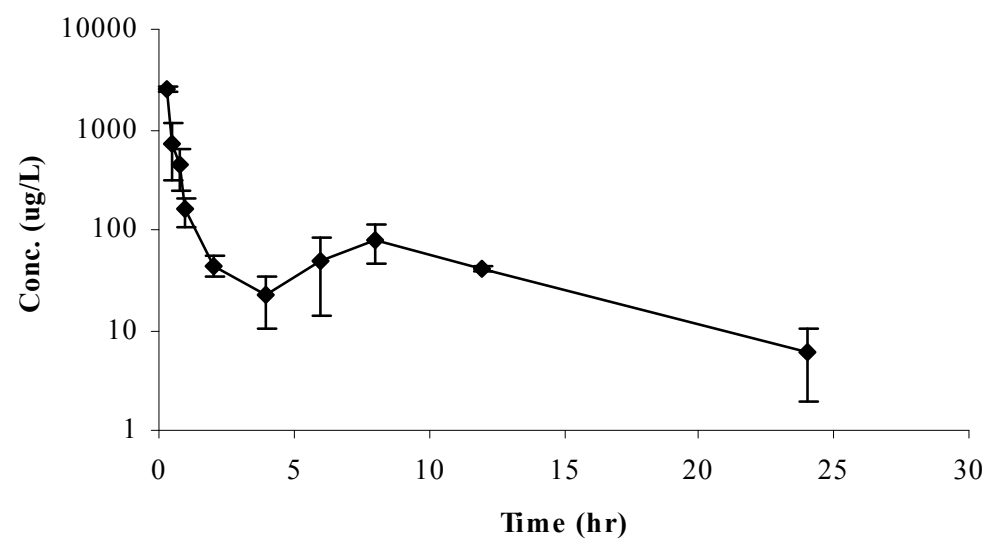

Figure 2-2. Measured Plasma Concentration-Time Profiles (Mean \pm SD) after Intravenous (10 mg/kg, Panel A) or Oral (100 mg/kg, Panel B) Administration of Lee 562 to Rats ( $\mathrm{n}=5-6$ per group). 
Table 2-1. $\quad$ Pharmacokinetic Parameters (Mean \pm SD) of Lee 562, Lee 878, Lee 952, and Lee 1106.

\begin{tabular}{|c|c|c|c|c|c|c|c|c|}
\hline \multirow{2}{*}{ Route } & \multirow{2}{*}{ Compound } & \multirow{2}{*}{$\begin{array}{c}\mathbf{t}_{1 / 2} \\
(\mathrm{hr})\end{array}$} & \multirow{2}{*}{$\begin{array}{c}\mathbf{A U C}_{\mathbf{i n f}} \\
(\mu \mathrm{g} \mathrm{hr} / \mathrm{L})\end{array}$} & \multirow{2}{*}{$\begin{array}{c}\text { Volume of } \\
\text { Distribution } \\
(\mathrm{L} / \mathrm{kg}) \\
\end{array}$} & \multirow{2}{*}{$\begin{array}{c}\text { Clearance } \\
(\mathrm{L} / \mathrm{hr} / \mathrm{kg})\end{array}$} & \multicolumn{2}{|c|}{ \% Dose Excreted } & \multirow{2}{*}{$\begin{array}{c}\text { Bioavailability } \\
(\%)\end{array}$} \\
\hline & & & & & & Urine & Feces & \\
\hline \multirow[t]{4}{*}{ IV } & Lee 562 & $1.30 \pm 0.89$ & $956 \pm 442$ & $28.9 \pm 29.4$ & $12.9 \pm 7.6$ & $0.001 \pm 0.0003$ & $0.001 \pm 0.001$ & \\
\hline & Lee 878 & $2.63 \pm 0.35$ & $19091 \pm 1724$ & $2.00 \pm 0.36$ & $0.527 \pm 0.05$ & ND & ND & \\
\hline & Lee 952 & $7.34 \pm 2.27$ & $1846 \pm 82.2$ & $58.0 \pm 20.1$ & $5.42 \pm 0.24$ & ND & ND & \\
\hline & Lee 1106 & $10.3 \pm 1.41$ & $22423 \pm 4096$ & $6.72 \pm 1.10$ & $0.456 \pm 0.07$ & $0.035 \pm 0.01$ & $0.14 \pm 0.06$ & \\
\hline \multirow[t]{4}{*}{ Oral } & Lee 562 & $3.69 \pm 0.86$ & $1519 \pm 458$ & $386 \pm 187$ & $71.6 \pm 23.2$ & $0.009 \pm 0.02$ & $0.023 \pm 0.02$ & $15.9 \pm 4.8$ \\
\hline & Lee 878 & ND & $52324 \pm 14834^{\mathrm{a}}$ & ND & $2.07 \pm 0.71^{\mathrm{b}}$ & ND & ND & $27.4 \pm 7.8^{\mathrm{b}}$ \\
\hline & Lee 952 & $3.39 \pm 1.16^{\mathrm{c}}$ & $2936 \pm 1119$ & $192 \pm 127$ & $37.7 \pm 12.5$ & ND & ND & $15.9 \pm 6.1$ \\
\hline & Lee 1106 & $9.24 \pm 1.71$ & $10230 \pm 9101$ & $231 \pm 174$ & $16.0 \pm 10.3$ & $0.025 \pm 0.02$ & $11.8 \pm 7.09$ & $4.6 \pm 4.1$ \\
\hline
\end{tabular}

ND: Not determined.

a: $\mathrm{AUC}_{0-48 \mathrm{hr}}$.

b: $\mathrm{AUC}_{0-48 \mathrm{hr}}$ was used for calculation of clearance and oral bioavailability.

c: Not reliable. Determined to limited log-linear phase. 
administration suggesting that the high clearance is the result of extensive metabolism. The high clearance resulted in a short terminal half-life of $1.3 \mathrm{hr}$ after IV and $3.7 \mathrm{hr}$ after oral administration, and an oral bioavailability of only $15.9 \%$. To investigate the extent of metabolism, Lee 562 was incubated in rat liver microsomal preparations. The microsomal metabolism of Lee 562 was rapid and extensive, with nearly complete loss $(>99.5 \%)$ of the parent compound within 90 minutes of incubation.

\section{Microsomal Metabolic Stability of Second Generation Compounds Lee 878 and Lee 952}

Metabolic stability of a compound is investigated to determine metabolic half-life and intrinsic clearance which can be major determinants for in vivo drug efficacy, and to aid in structural optimization of lead compounds in drug discovery settings [197]. Analysis of the structure of Lee 562 led us to attribute its metabolic instability in part to the amide bond of the compound. Thus, two follow-up second generation compounds, Lee 878 and Lee 952, were tested that included structural modifications to improve metabolic stability. For Lee 878 , the amide bond was replaced by an isoxazole ring; for Lee 952, the amide hydrogen was substituted with a methyl group (Figure 2-1). The microsomal metabolism of Lee 952 was rapid and extensive, with $>95 \%$ loss of the parent compound after 90 minutes of incubation (4.3\% remaining). The metabolic halflife appears to be less than 15 minutes. The desired cutoff for metabolic stability in most preclinical programs is more than $30 \%$ of parent remaining stable after 90 minutes of incubation [198]. In contrast, Lee 878 exhibited acceptable metabolic stability under this criterion with $31.4 \%$ of the parent compound remaining stable at the end of incubation.

\section{Pharmacokinetics of Second Generation Compounds Lee 878 and Lee 952}

The plasma concentration-time profiles and pharmacokinetic parameters of Lee 878 and Lee 952 after single IV and oral administration to rats are shown in panels A and B of Figures 2-3 and 2-4 and Table 2-1, respectively. Oral absorption of Lee 878 was slow with peak plasma concentration $\left(\mathrm{C}_{\max }\right)$ occurring about 6 hours after administration. In contrast, oral absorption of Lee 952 was rapid with peaks observed within 15 minutes of administration. Lee 952 distributed more extensively in the body than Lee 878 with a distribution volume of $58 \mathrm{~L} / \mathrm{kg}$ compared to $2.0 \mathrm{~L} / \mathrm{kg}$. As expected from the microsomal metabolic stability studies, the mean plasma clearance was much larger for Lee 952 with $5.4 \mathrm{~L} / \mathrm{hr} / \mathrm{kg}$ compared to $0.53 \mathrm{~L} / \mathrm{hr} / \mathrm{kg}$ for Lee 878 . Consequently, oral bioavailability of Lee 878 was found to be $27.4 \%$, while Lee 952 exhibited poor oral bioavailability with only $15.9 \%$. Since the terminal elimination phase was not captured after oral administration of Lee $878, \mathrm{AUC}_{0-48} \mathrm{hr}$ was used to determine oral bioavailability. Despite its substantially lower clearance, Lee 878 exhibited a much shorter terminal half-life of $2.6 \mathrm{hr}$ after IV administration compared to Lee 952 with $7.3 \mathrm{hr}$. This can be explained with Lee 878's substantially smaller distribution volume compared to Lee 952, which indicates less drug distribution to peripheral tissues and thus easier access of drug 
A)

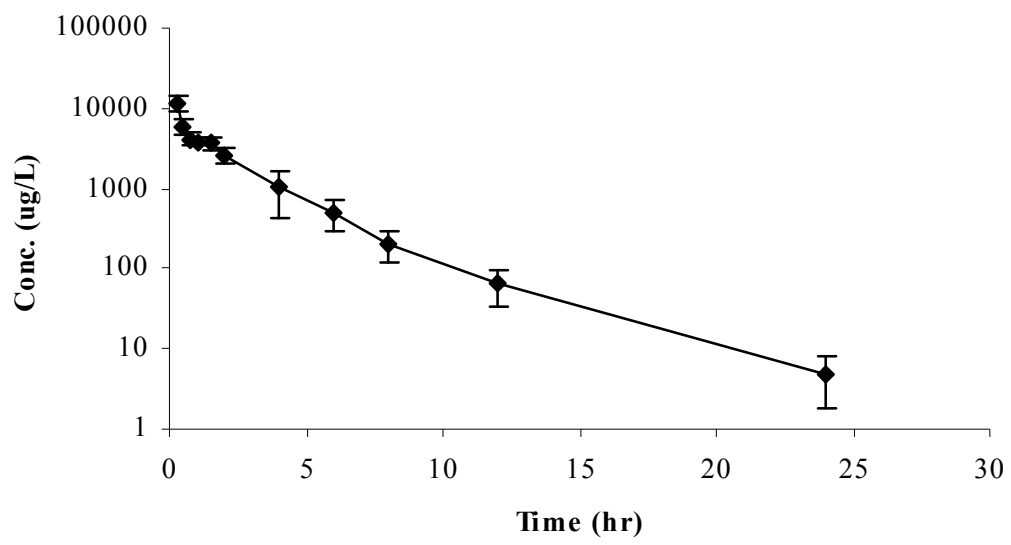

B)

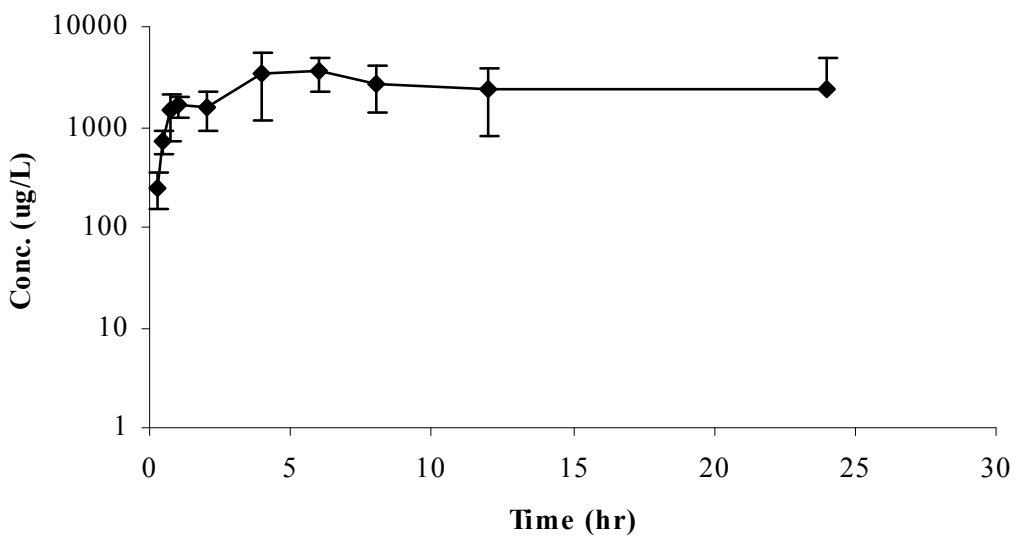

Figure 2-3. Measured Plasma Concentration-Time Profiles (Mean \pm SD) after Intravenous (10 mg/kg, Panel A) or Oral (100 mg/kg, Panel B) Administration of Lee 878 to Rats ( $\mathrm{n}=5-6$ per group). 
A)

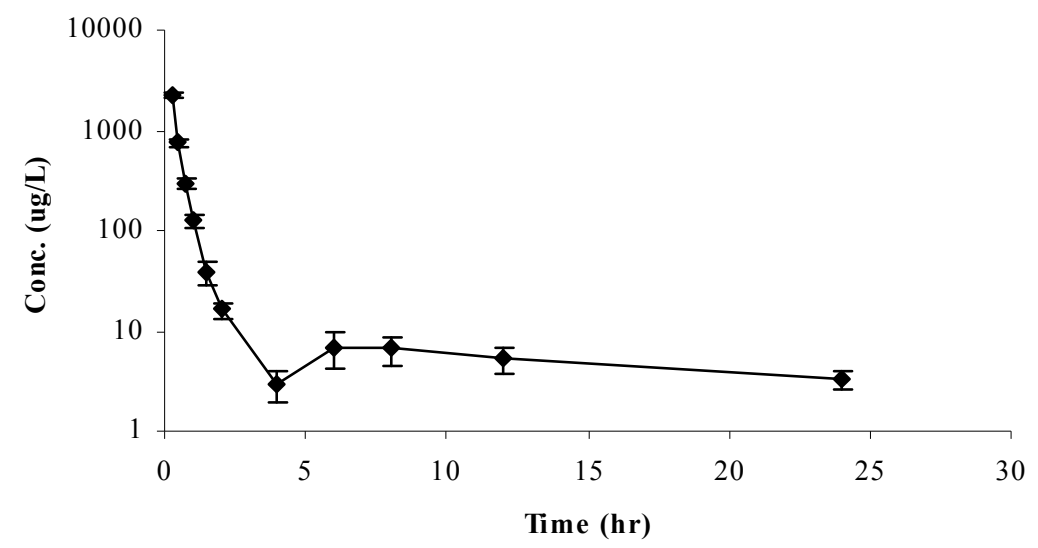

B)

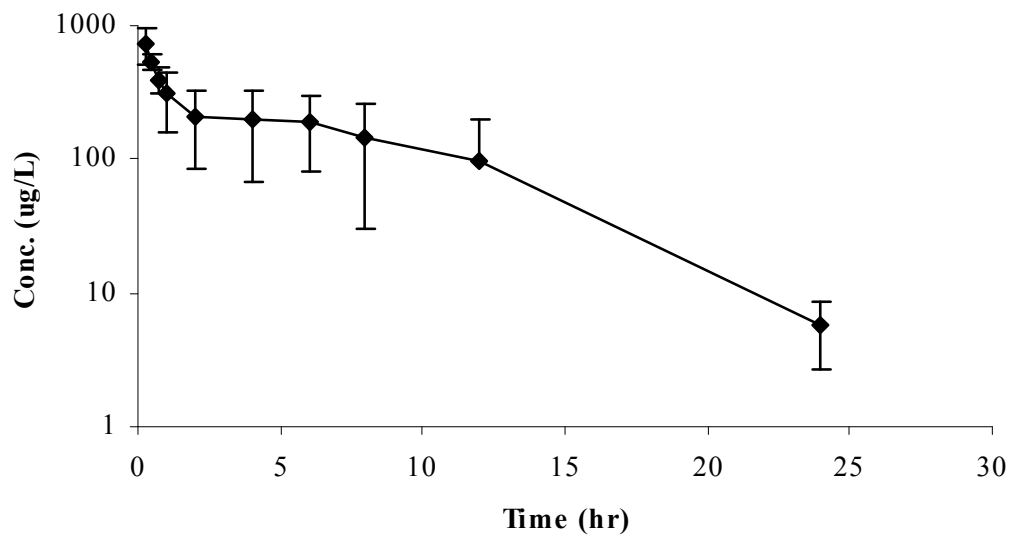

Figure 2-4. Measured Plasma Concentration-Time Profiles (Mean \pm SD) after Intravenous (10 mg/kg, Panel A) or Oral (100 mg/kg, Panel B) Administration of Lee 952 to Rats ( $\mathrm{n}=5-6$ per group). 
compound to elimination processes.

\section{Metabolic Stability of Third Generation Compounds}

Since the microsomal metabolic stability was found to be correlated with the oral bioavailability of Lee 562, Lee 878 and Lee 952, a new set of nine compounds within the nitrofuranylamide series was screened for metabolic stability. Chemical structures of the compounds and microsomal metabolic stability data are shown in Figure 2-5. Lee 1106 exhibited superior metabolic stability with more than $70 \%$ of the parent compound remaining stable after 90 minutes of incubation. Thus, Lee 1106 was selected for further pharmacokinetic evaluation in rats. The structural modifications of Lee 562 that lead to the other three compounds, Lee 878, Lee 952 and Lee 1106, are shown in Figure 2-1.

\section{Pharmacokinetics of the Third Generation Compound Lee 1106}

The plasma concentration-time profiles of Lee 1106 after single IV and oral administration are shown in panels A and B of Figure 2-6, the corresponding pharmacokinetic parameters in Table 2-1. Absorption of Lee 1106 was slow, with peak plasma concentration $\left(\mathrm{C}_{\max }\right)$ observed $9 \mathrm{hr}$ after administration. Oral bioavailability of the compound was $4.6 \%$. Lee 1106 showed a distribution volume of $6.7 \mathrm{~L} / \mathrm{kg}$. As expected from the metabolic stability data, the plasma clearance of the compound was low, 0.46 $\mathrm{L} / \mathrm{hr} / \mathrm{kg}$. The unchanged compound excreted in urine was less than $1 \%$ of the dose by both routes of administration, whereas about $0.1 \%$ and $12 \%$ of the dose was excreted unchanged in feces after IV and oral administration, respectively. The low plasma clearance of Lee 1106 resulted in a longer terminal half-life than observed in the other tested nitrofuranylamides, $10.3 \mathrm{hr}$ after the IV and $9.24 \mathrm{hr}$ after the oral administration.

\section{Solubility Screening}

Aqueous solubility is one of the most important properties for a compound intended to be developed as an orally active molecule. The solubility of the investigated compounds is presented in the Table 2-2. A compound is conventionally classified as highly soluble when the largest dose of the compound is soluble in less than $250 \mathrm{~mL}$ water over a $\mathrm{pH}$ range from 1.0 to 7.5 [46]. Though there is no strict cutoff value for solubility available, solubility above $50 \mu \mathrm{g} / \mathrm{mL}$ is arbitrarily considered acceptable for compound progression in most preclinical development programs [12]. It is assumed that this level of solubility would never pose a problem in limiting bioavailability. Lee 562, Lee 878 and Lee 1106 did not pass this solubility requirement, but the solubility for Lee 952 clearly exceeds the cutoff value. Predicted octanol-water partition coefficient, clogP values for Lee 562, Lee 878, Lee 952 and Lee 1106 are shown in Table 2-2. A graph showing the relationship between the clogP and the aqueous solubility of all the tested compounds is presented in the panel A of Figure 2-7. 

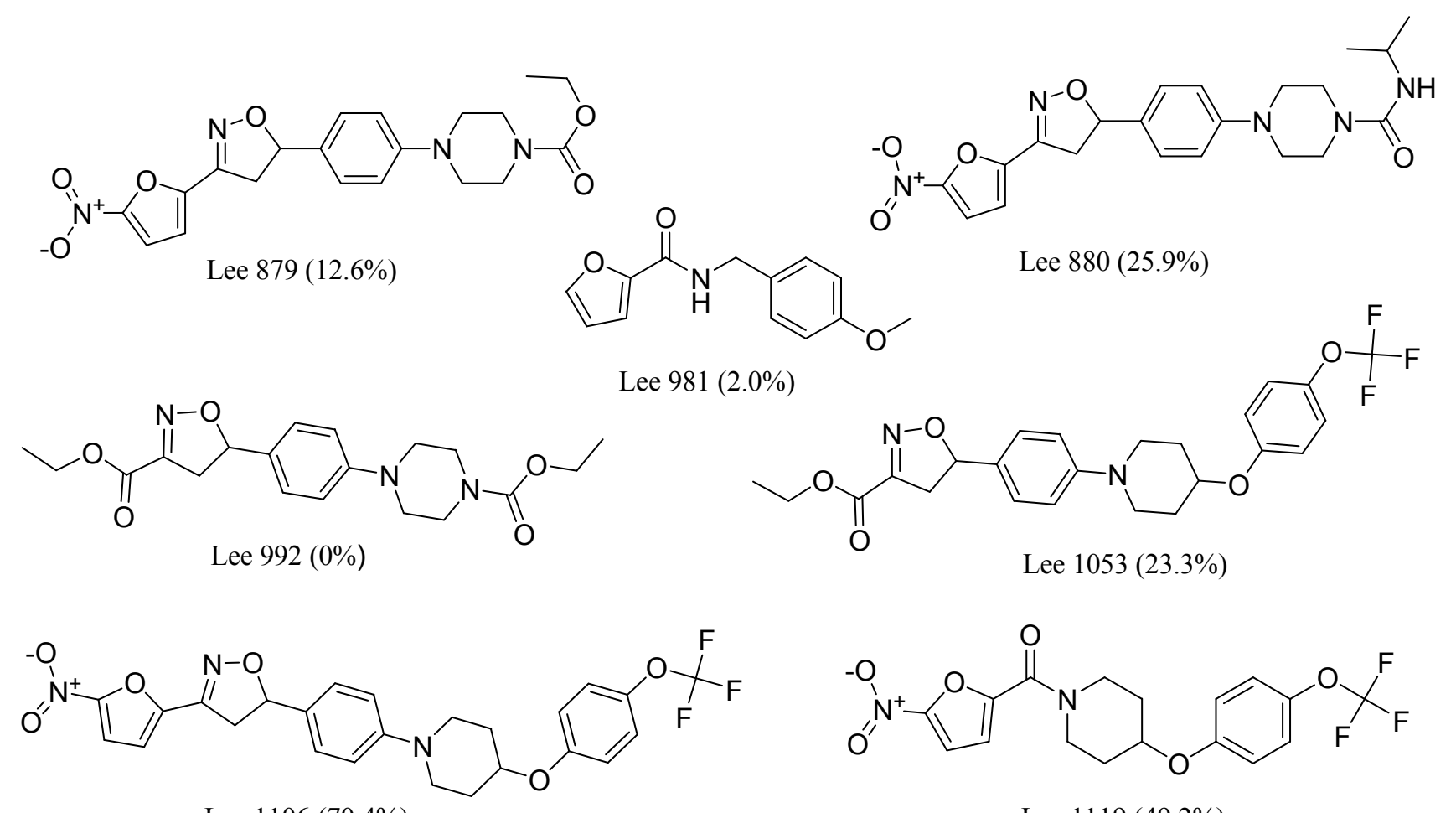

Lee $1106(70.4 \%)$

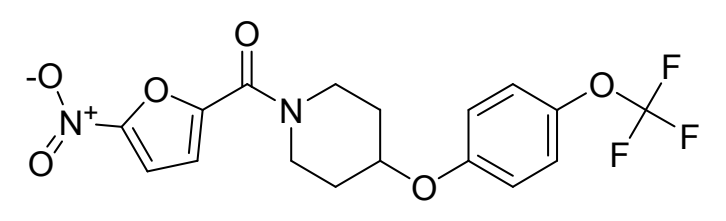

Lee $1119(49.2 \%)$

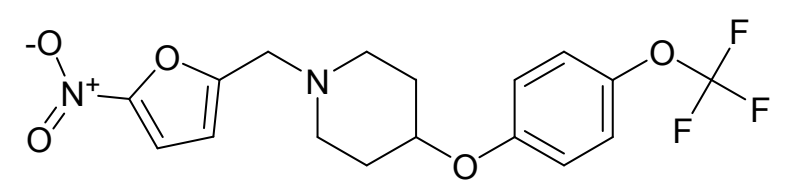

Lee $1120(0.5 \%)$

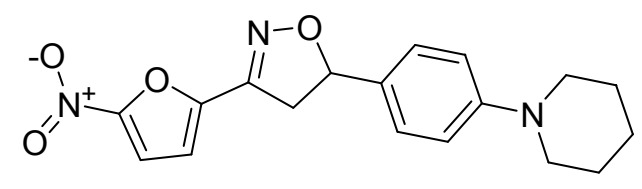

Lee $1131(6.2 \%)$

Figure 2-5. Chemical Structures of Third Generation Nitrofuranylamides. The values provided in parenthesis indicate the metabolic stability in an in vitro microsomal stability assay (percent parent compound remaining stable after 90 min of incubation). 
A)

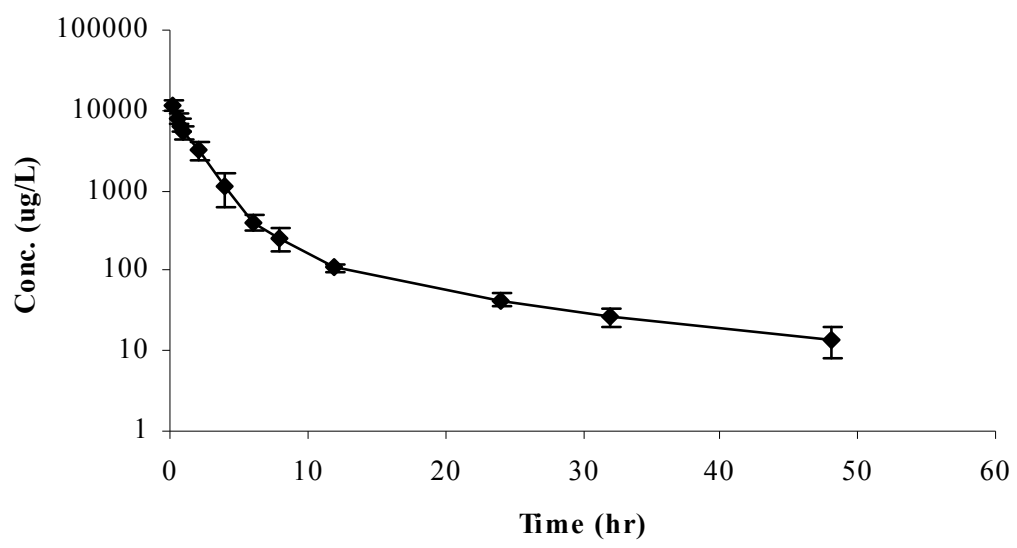

B)

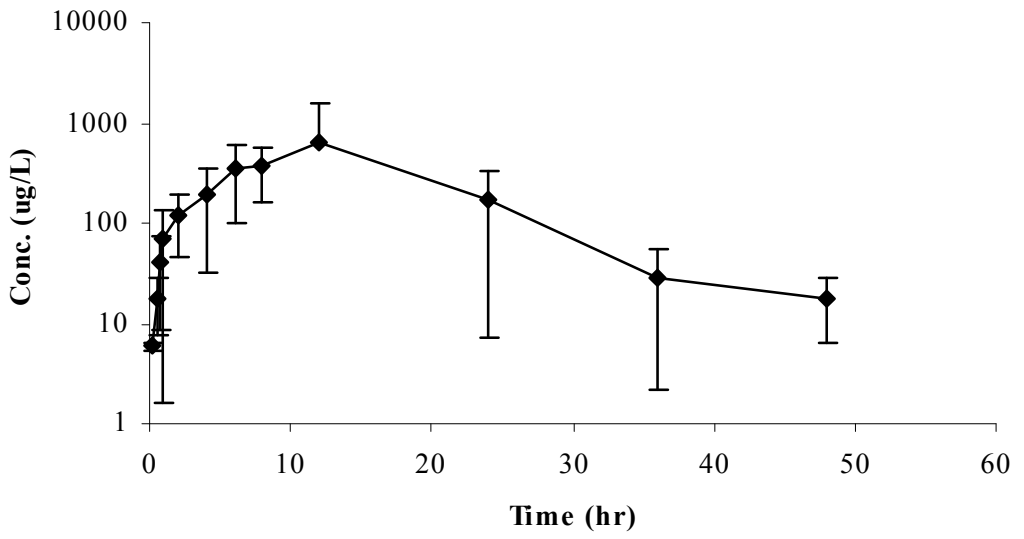

Figure 2-6. Measured Plasma Concentration-Time Profiles (Mean \pm SD) after Intravenous (10 mg $/ \mathrm{kg}$, Panel A) or Oral (100 mg/kg, Panel B) Administration of Lee 1106 to Rats ( $\mathrm{n}=5-6$ per Group). 
Table 2-2. Aqueous Solubility and Plasma Protein Binding of Lee 562, Lee 878, Lee 952, and Lee 1106.

\begin{tabular}{ccccccc}
\hline Compound & $\begin{array}{c}\text { Molecular } \\
\text { Weight }\end{array}$ & $\begin{array}{c}\text { MIC90* } \\
(\boldsymbol{\mu g} / \mathbf{m L})\end{array}$ & clogP & \multicolumn{2}{c}{ Solubility (mg/L) } & \multirow{2}{*}{$\begin{array}{c}\text { \% } \\
\text { Protein } \\
\text { Binding }\end{array}$} \\
\hline Lee 562 & 421.4 & 0.0062 & 2.41 & 18.5 & 4.43 & 96.43 \\
Lee 878 & 432.5 & 0.00005 & 4.29 & 3.34 & 0.24 & 99.92 \\
Lee 952 & 389.4 & 0.09 & 1.91 & 1975 & 759 & 44.53 \\
Lee 1106 & 517.5 & 0.025 & 6.41 & BLQ $^{\mathbf{a}}$ & 2.67 & 99.99 \\
\hline
\end{tabular}

* $\mathrm{MIC}_{90}$ : Minimum inhibitory concentration to inhibit the growth of $90 \%$ of organisms determined against $M$. tuberculosis $\mathrm{H}_{37} \mathrm{Rv}$ using microbroth dilution in Middlebrook $7 \mathrm{H} 9$.

a: BLQ: Below the limit of quantification. 
A)

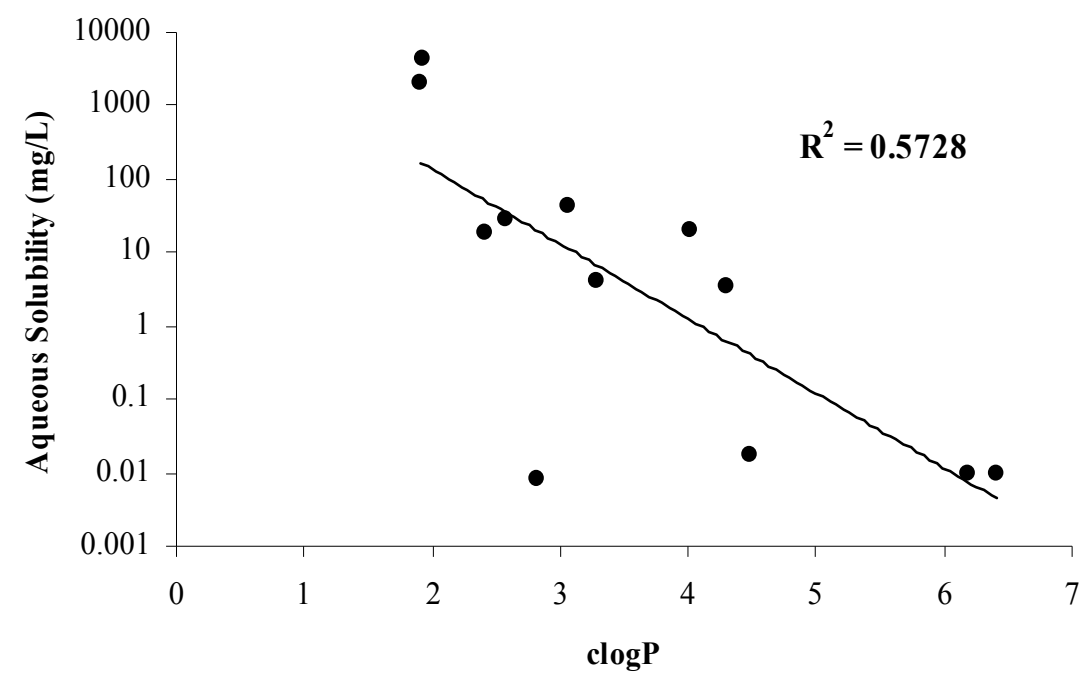

B)

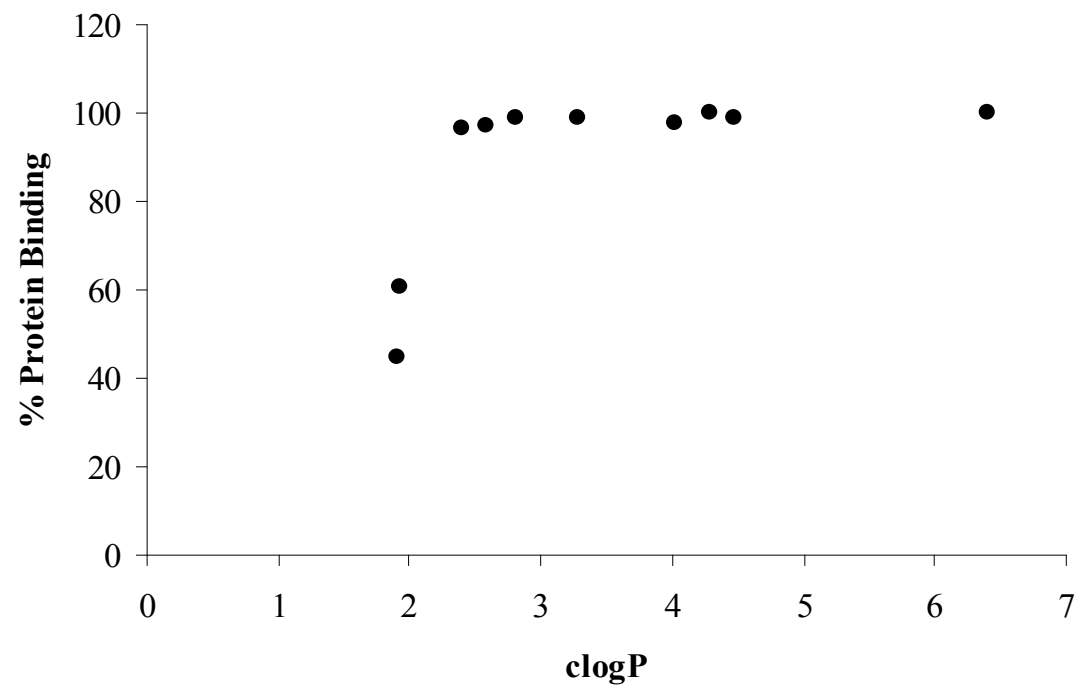

Figure 2-7. Relationship between Aqueous Solubility, Plasma Protein Binding and clogP in the Investigated Nitrofuranylamides. Panel A shows correlation between aqueous solubility and $\operatorname{cog} \mathrm{P}$ and panel $\mathrm{B}$ shows correlation between plasma protein binding and $\operatorname{cog} \mathrm{P}$. The plasma protein binding of the compounds Lee 992 and Lee 1053 could not be determined due to instability in plasma. 


\section{Plasma Protein Binding}

The compounds Lee 562, Lee 878 and Lee 1106 are extensively bound to plasma proteins with $96.4 \%, 99.9 \%$ and $>99.9 \%$ binding, respectively (Table $2-2$ ). In contrast, Lee 952 was only $44.5 \%$ bound to plasma proteins. A graph showing relationship between clogP and the protein binding of all the tested compounds is presented in the panel B of Figure 2-7.

\section{Discussion}

The first tested lead compound, Lee 562, exhibited a high systemic clearance, short terminal half-life, and low oral bioavailability of $15.9 \%$. These observations were further supported by the poor metabolic stability of Lee 562, which was attributed to its amide linkage and the benzyl piperazine side chain in the structure. Thus, two second generation follow-up compounds were tested that included structural modifications for increased metabolic stability. For Lee 878 , the amide bond was replaced by an isoxazole ring; for Lee 952, the amide hydrogen was substituted with a methyl group and the benzyl piperazine was replaced with a solubilizing morpholino group. Both compounds showed improved metabolic stability in the rat microsome assay compared to Lee 562, with Lee 878 being much more stable than Lee 952. As expected, this in vitro observation translated into an increased in vivo stability (lower clearance) of Lee 878 compared to Lee 562 and Lee 952, with a 20- and 10-fold higher systemic exposure, respectively. As a consequence, oral bioavailability of Lee 878 reached $\sim 27 \%$ compared to $16 \%$ for Lee 952 and Lee 562.

The low oral bioavailability of Lee 562 and Lee 952 was predictable, considering that the mean plasma clearance after IV administration was $12.9 \mathrm{~L} / \mathrm{hr} / \mathrm{kg}$ and $5.4 \mathrm{~L} / \mathrm{hr} / \mathrm{kg}$ respectively. Based on their extensive metabolism, their plasma clearance and a typical hepatic blood flow of $3.3 \mathrm{~L} / \mathrm{hr} / \mathrm{kg}$ in rats [199], the theoretical hepatic extraction ratio (ER) for these compounds could be estimated as 3.9 and 1.6 for Lee 562 and Lee 952, respectively. As the maximum physiologically possible hepatic ER is 1, i.e., complete removal and/or metabolic conversion of all drug presented to the liver, the calculated ERs for Lee 562 and Lee 952 exceeding 1 suggest the presence of extrahepatic metabolism and/or sequestration of drug into blood cells. Upon incubation with heparinized human whole blood, Lee 562 showed slow distribution into red blood cells with a partitioning coefficient of 2.1 (results not shown). Overall, the low oral bioavailability of these compounds could be attributed to high first-pass metabolism. For Lee 562, poor aqueous solubility apart from the first-pass inactivation could also be a contributing factor to its low oral bioavailability.

Similarly, assuming that clearance of Lee 878 is only hepatic, the hepatic ER for Lee 878 could be estimated as 0.16 . Considering first-pass inactivation alone, this would suggest that the maximum oral bioavailability for Lee 878 could theoretically reach $84 \%$ $\left([1-E R]^{*} 100\right)$ as opposed to the observed bioavailability of $27.4 \%$. Possible reasons for this difference in bioavailability could be poor aqueous solubility, transporter-mediated 
efflux in the gastrointestinal membrane, and/or degradation/metabolism in the gastrointestinal tract.

The elimination half-life of Lee 878 was only $2.6 \mathrm{hr}$, mainly due to the surprisingly small volume of distribution of this compound. Considering the high clogP of 4.3 for Lee 878 and the corresponding low aqueous solubility, the limited volume of distribution of Lee 878 is most likely explained by its excessive binding $(99.92 \%)$ to plasma proteins. A high plasma protein binding reduces the free, pharmacologically active concentrations of a drug and limits its tissue distribution. This might be the major reason why Lee 878 was found to have low in vivo efficacy in a mouse model of M.tb infection despite favorable systemic exposure, high metabolic stability and high in vitro activity [192].

Since the microsomal metabolic stability was found to be correlated with the oral bioavailability of the first and second generation compounds we tested, a new set of nine third generation compounds belonging to the same series were screened for microsomal metabolic stability to guide selection of a stable compound for pharmacokinetic evaluation.

As Lee 1106 exhibited superior metabolic stability, its pharmacokinetic properties were evaluated in vivo. The compound exhibited favorable pharmacokinetic properties such as a low systemic clearance and a long terminal half-life. However, oral bioavailability of Lee 1106 is poor despite a mean plasma clearance after IV administration of only $0.46 \mathrm{~L} / \mathrm{hr} / \mathrm{kg}$. Assuming that all clearance for Lee 1106 is mediated by the liver, the hepatic ER for Lee 1106 would be calculated as 0.14 and the maximum oral bioavailability based on first-pass inactivation alone would be $86 \%$. However, Lee 1106 exhibited a very low oral bioavailability of $4.6 \%$. Poor aqueous solubility of the compound could explain to some extent the observed low oral bioavailability of the compound, which might be overcome by optimizing the dosage form using solubilizing excipients [200]. Further, possible degradation/metabolism and/or transporter-mediated efflux of the compound in the gastrointestinal tract could also contribute to this observation. In addition, poor aqueous solubility, high plasma protein binding and high clogP of Lee 1106 suggest hydrophobicity of the molecule. Lee 1106 has a molecular weight exceeding $500 \mathrm{Da}(517.5 \mathrm{Da})$ and a clogP exceeding $5(6.41)$, thereby clearly deviating from the Lipinski's rule of five, a scoring function used to predict orally active molecules [47].

A closer look at the structures of the compounds suggests that Lee 878 and Lee 1106 have a larger number of aromatic rings imparting more hydrophobicity to the molecule which in turn decreases their aqueous solubility. Lee 952 has fewer aromatic rings in its structure and exhibits high aqueous solubility. As shown in the panel A of Figure 2-7, aqueous solubility in our set of compounds was inversely correlated with clogP as measure of hydrophobicity ( $\mathrm{p}=0.004$, Pearson correlation coefficient: -0.76$)$. Similarly, protein binding was consistently above $96 \%$ with higher clogP values in all the tested compounds (Figure 2-7, panel B). 
Based on these observations, aqueous solubility and metabolic stability are the most important determinants of oral bioavailability of the nitrofuranylamide compounds. Thus, an early evaluation of aqueous solubility, and protein binding coupled with metabolic stability could be useful for predicting the oral bioavailability of novel nitrofuranylamide compounds. Accordingly, a screening paradigm was proposed for lead optimization of compounds of the nitrofuranylamide class (Figure 2-8). As a first step in this process, compounds will be designed for synthesis using in silico approaches such as quantitative structure activity relationships (QSAR) to predict antimicrobial efficacy. In the next step, a variety of in silico predictions (quantitative structure-property

relationships) such as aqueous solubility, metabolic stability, protein binding and clogP will be used for rank-ordering the compounds for synthesis. The most promising compounds will then be synthesized and tested for in vitro activity against M.tb. Potent compounds resulting from the in vitro activity screen will be studied for aqueous solubility and in vitro pharmacokinetic properties such as metabolic stability and protein binding. The compounds with higher stability, higher aqueous solubility and lower protein binding will be selected for pharmacokinetic and bioavailability evaluation in rats. The compounds that exhibit high oral bioavailability and long elimination half-life in rats will be progressed into more expensive and time-consuming in vivo evaluations of activity in animal models such as mouse models of $M$.tb infection. The information obtained at each step will be used for updating the design of compounds for synthesis and the filtering used at each step will allow concentrating development resources on a smaller number of more promising compounds which makes the lead optimization process more efficient.

\section{Conclusion}

In summary, we determined the pharmacokinetic properties of key compounds in the nitrofuranylamide series and developed a screening paradigm that could be useful for the further optimization of leads in the group of anti-infectives against M.tb. This paradigm may also be useful for lead optimization of other series of anti-infectives. 

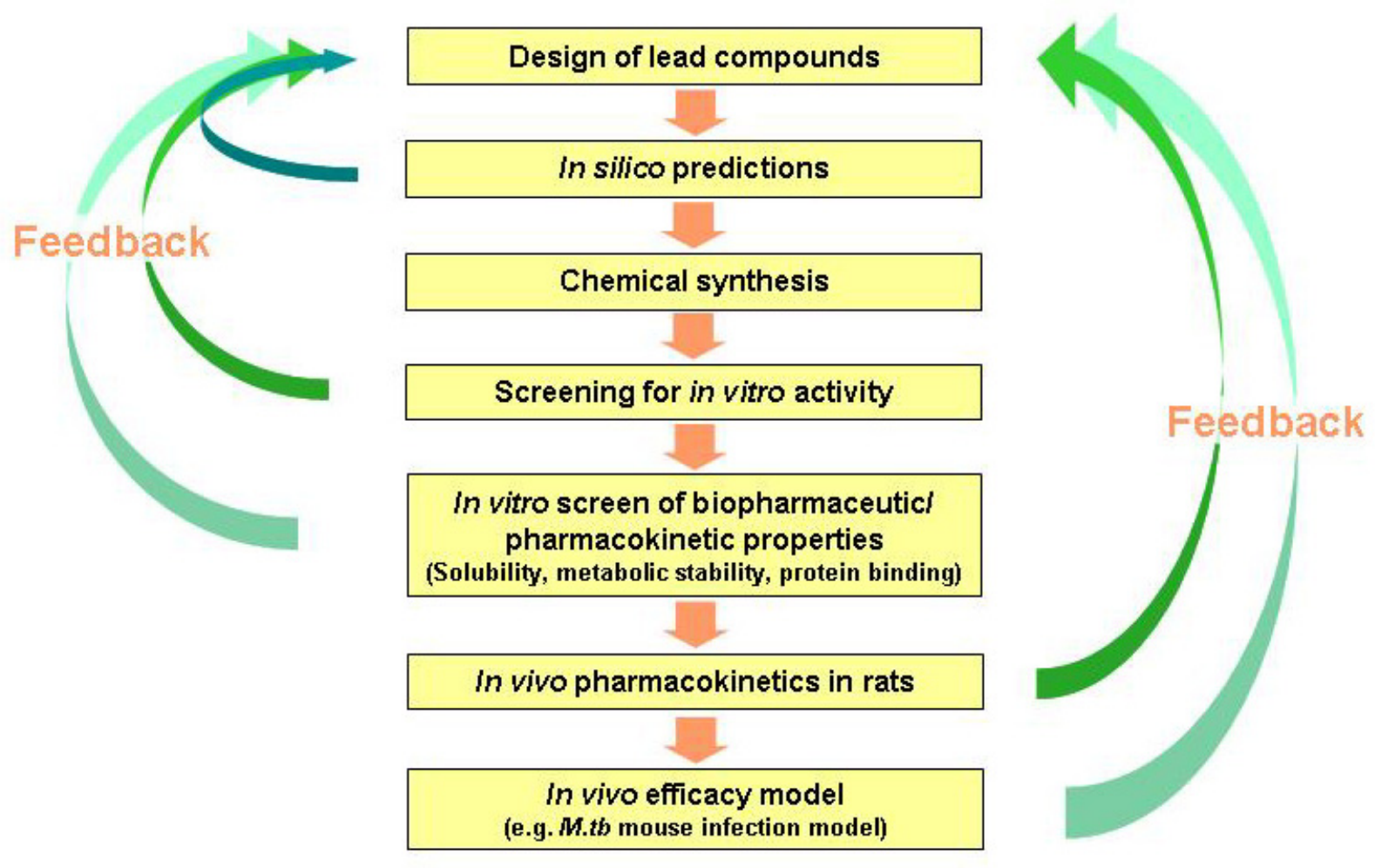

Figure 2-8. Screening Paradigm for Lead Optimization of Anti-TB Compounds. 


\section{CHAPTER 3. AN IN VITRO PK/PD MODEL TO DETERMINE TIME-KILL CURVES OF ANTIBIOTICS AGAINST SLOW-GROWING MICROORGANISMS}

\section{Introduction}

In spite of the fact that bacteria are exposed to dynamically changing drug concentrations in vivo, antibiotics are frequently dosed based on point estimates of efficacy derived from exposure to constant concentrations. Until last decade, it was a common practice to select antimicrobial dosing regimens above the minimum inhibitory concentration (MIC) of the pathogen being treated. This approach, however, has several disadvantages as it does not accurately reflect the complex interaction among the three major players, the drug, the microorganism and the host. Constant (static) drug levels used in MIC determinations hardly reflect the actual in vivo situation where the pharmacodynamic effect is the result of dynamic exposure of the organism to the unbound drug fraction in the target tissue. Furthermore, the MIC provides only limited information on the kinetics of bactericidal activity as it is used as a threshold value for the activity of the antibiotic. In reality, however, antimicrobials usually have already some activity at concentrations below the MIC and may not have the maximum activity just above the MIC. Thus, complete time courses of the drug effect in response to concentration-time courses encountered in vivo seem to be more adequate and informative for selecting optimal dosing strategies than the use of point estimates such as MIC [201].

In recent years, integrated pharmacokinetic/pharmacodynamic (PK/PD) approaches have been developed as a more sophisticated methodology to assess the efficacy of antimicrobials based on dynamic kill curves. These PK/PD-based approaches are being used to help in the selection of potential drug candidates and to provide a scientific rationale for selecting dosing regimens that increase the efficacy and reduce the probability of emergence of drug resistance [176]. The US Food and Drug Administration and the European Medicines Evaluation Agency have been advocating the use of various in vitro and in vivo models to characterize the PK/PD properties of antibiotics and to predict the efficacy of candidate compounds in the early stages of the drug development process [202, 203].

In vitro $\mathrm{PK} / \mathrm{PD}$ models can be used to study the antibacterial effect of single and combination drug compounds and dosing regimens before in vivo efficacy studies [204]. The advantage of these models is that the appropriate human/animal pharmacokinetic profiles can easily be simulated and the effect of these changing drug concentrations on bacterial growth (i.e., via kill curves) and emergence of resistance can be assessed.

Different species of microorganisms and clinical isolates of the same bacteria that vary in sensitivity to drug treatment can be exposed to different dosing regimens, such that the PD parameters can be identified that best explain the activity/efficacy of the drug and allow the optimization of dosage regimens for maximum efficacy. Since the influence of the immune system is absent in these in vitro models, the drug-bacterium interactions can 
be measured more precisely. Thus, in vitro models offer a safer and more ethical way of assessing the PK/PD relationships of antibiotics compared to animal or human studies [201, 205-207].

Several in vitro $\mathrm{PK} / \mathrm{PD}$ models for antibiotics have been reported in the literature and can be broadly classified as either dilution models or diffusion models based on the mechanism by which the dynamic concentration-time profiles are simulated [204, 208]. However, most of the available in vitro models have been applied for fast growing bacteria and are not suitable for studies on slow growing microorganisms such as Mycobacterium tuberculosis. The previously reported dilution models without a filter membrane [209-211] are not suitable for slow growing organisms since the bacteria are washed out during the dilution process and the bacterial replication rate is not sufficient to offset this loss. The reported dilution models with filter membrane have so far not been utilized for slow growing organisms in the published literature. More recently, several reports have been published using hollow fiber bioreactors (diffusion models) as in vitro models for testing antibacterial activity against $M$. tuberculosis [212-215]. However, there are severe limitations associated with the use of hollow fiber bioreactors for in vitro culturing of bacteria. As these bioreactors are complex and difficult to sterilize between experiments, new hollow fiber cartridges are recommended for every study which makes a broad-based application of these experiments cost-prohibitive. Further, nonspecific drug binding to the hollow fiber capillaries, especially of the highly lipophilic compounds frequently tested for anti-mycobacterial activity, can produce erroneous results. In view of these limitations of the existing in vitro models, we decided to develop a novel in vitro $\mathrm{PK} / \mathrm{PD}$ model for studying the pharmacologic properties of antibiotics against slowgrowing microorganisms that is simple, inexpensive and easy to construct and operate. In the following, we illustrate the application of this model to determine time-kill curves of slow growing microorganisms by characterizing the effect of isoniazid (INH) dosing regimens on Mycobacterium bovis Bacillus Calmette-Guerin (BCG).

\section{Materials and Methods}

\section{In vitro PK/PD Model Design}

The in vitro $\mathrm{PK} / \mathrm{PD}$ model system consisted of a two-armed, water jacketed spinner culture flask (1965 series spinner flask, Bellco Glass, Vineland, NJ) as depicted in Figure 3-1. A $25 \mathrm{~mm}$ diameter filter unit (Pall Corporation, East Hills, NY) was fixed to one end of a custom made hollow steel tube. The other end of the steel tube was connected to plastic tubing (PharMed tubing, Cole-Parmer, Vernon Hills, IL). A prefilter ( $5 \mu \mathrm{m}$, Millipore, Billerica, MA) and filter membrane $(0.22 \mu \mathrm{m}$, Millipore, Billerica, MA) were placed in the filter unit to prevent leakage of bacteria during the dilution process. The whole filter unit was suspended into the media from the top. The flask was placed on a magnetic stirrer; a magnetic stir bar in the flask ensured homogeneity of the culture and prevented membrane pore blockage. One of the side arms covered with silicone septa was used for repeated sampling. The other sidearm was connected to a reservoir containing 


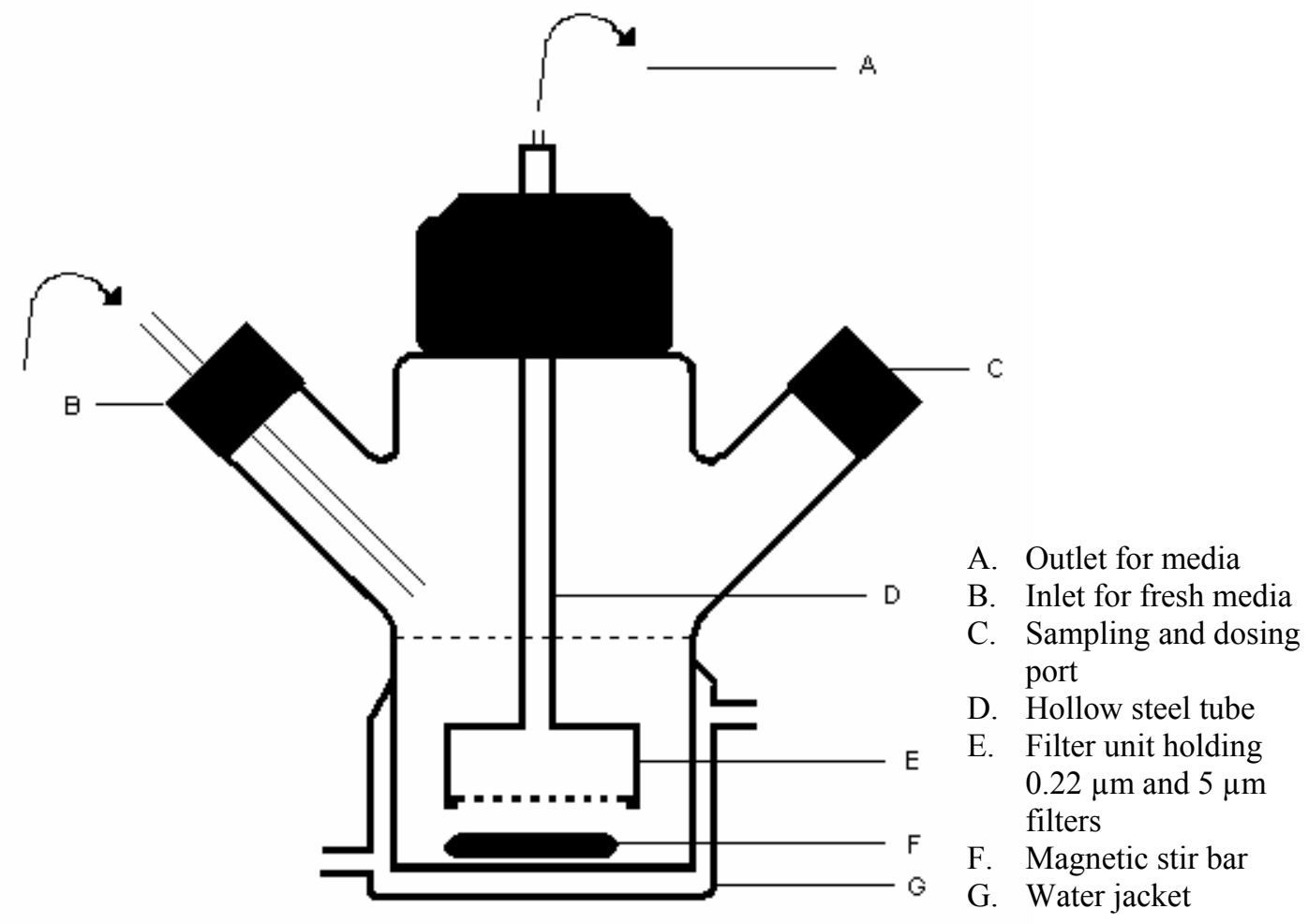

Figure 3-1. Schematic Diagram of In vitro PK/PD Model. 
antibiotic free sterile medium through a thin plastic tube (PharMed tubing, Cole-Parmer, Vernon Hills, IL). The outlet tubing from the filter unit was connected to a peristaltic pump (Masterflex L/S, Cole-Parmer, Vernon Hills, IL) to continuously withdraw the medium from the flask at a constant rate. Fresh sterile medium (Middlebrook 7H9, Becton Dickinson, Sparks, MD) was pumped into the flask at the same rate. The solution of the antibiotic INH was added through the sidearm. INH concentration in the flask was decreased exponentially according to the equation,

$$
C=C_{0} \cdot e^{-k \cdot t}
$$

where $C_{0}$ is the initial concentration of INH in the flask, $C$ is the concentration at any time $t, k$ is the elimination rate constant and $t$ is the time elapsed since the addition of INH. The elimination rate constant $k$ is equal to $F / V$, where $F$ is the flow rate of the medium and $V$ is the volume of the medium in the flask. Different half-lives $\left(t_{1 / 2}\right)$ of INH were simulated in the model by changing the flow rate according to the equation,

$$
F=V \cdot \frac{\ln 2}{t_{1 / 2}}
$$

The temperature in the flask was maintained at $37^{\circ} \mathrm{C}$ by attaching a thermostatic water circulator to the water jacket of the flask. The apparatus was sterilized by autoclaving between experiments and was kept in a biological safety cabinet during operation. Working picture of the in vitro model was shown in Figure 3-2.

\section{Culture, Media, and Antibiotics}

Mycobacterium bovis BCG was grown in Middlebrook $7 \mathrm{H} 9$ broth (Becton Dickinson, Sparks, MD) supplemented with $10 \%$ albumin dextrose complex and $0.1 \%$ v/v Tween 80 . The cultures were stored at $-80^{\circ} \mathrm{C}$ in Middlebrook $7 \mathrm{H} 9$ broth. For each experiment, cultures were thawed and incubated at $37^{\circ} \mathrm{C}$ in Middlebrook $7 \mathrm{H} 9$ broth for 4 days.

The antibiotic isoniazid (INH) was obtained from Sigma-Aldrich (St. Louis, MO). A stock solution of antibiotic was prepared in DMSO and stored at $-80^{\circ} \mathrm{C}$. Working solutions of INH were prepared from the stock solution using sterile water.

\section{Minimum Inhibitory Concentration of Isoniazid}

The MIC of INH was determined using the microbroth dilution method according to the CLSI guidelines [73] and was read by visual inspection. Two fold serial dilutions of INH in $100 \mu \mathrm{L}$ of the Middlebrook $7 \mathrm{H} 9$ broth medium were prepared in roundbottomed 96 well microtiter plates (Nunc, Sigma-Aldrich, St. Louis, MO). An equivalent volume $(100 \mu \mathrm{L})$ of broth containing $\sim 10^{5} \mathrm{CFU} / \mathrm{mL}$ bacteria was added to each well to give final concentrations of INH starting at $200 \mathrm{mg} / \mathrm{L}$ and the plates were incubated 


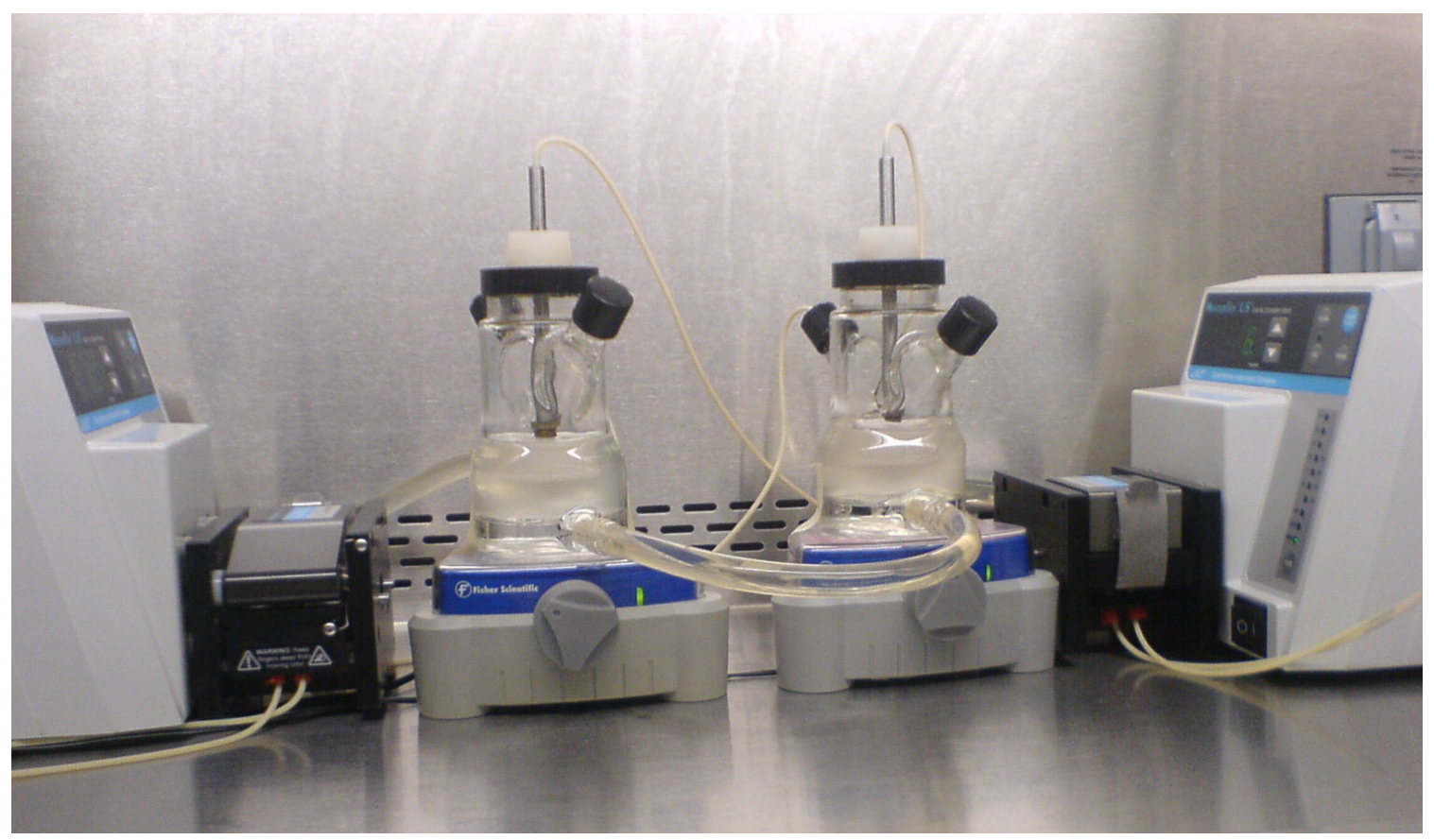

Figure 3-2. A Picture Showing Two Working Units of In vitro PK/PD Model.

aerobically at $37^{\circ} \mathrm{C}$ for 7 days. The MIC was recorded as the lowest concentration of drug that prevented visible growth.

\section{Time-Kill Curves of INH}

The test organism M. bovis BCG was used to evaluate the in vitro model. M. bovis BCG was grown to the early logarithmic growth phase in Middlebrook7H9 medium. The logarithmically growing culture was then used to inoculate the in vitro model. The final density of the culture in the in vitro system was $\sim 10^{6} \mathrm{CFU} / \mathrm{mL}$. The medium was drawn from the flask via the combination of a $5 \mu \mathrm{m}$ and a $0.22 \mu \mathrm{m}$ filter at a predetermined flow rate that mimicked the in vivo elimination half-life of INH. Fresh sterile medium was pumped into the flask at the same rate through one side arm to keep the volume of the medium at the initial level. The addition of INH doses was started after the culture had reached the logarithmic growth phase which was determined by the optical density of the culture. INH doses were added daily for 7 days to simulate INH plasma concentrationtime profiles in humans treated with 25,100 and $300 \mathrm{mg} /$ day (Table 3-1). Since INH is metabolized by a polymorphically expressed enzyme, NAT2 (N-acetyl transferase 2$)$, this difference in INH metabolizing activity results in a bimodal distribution of elimination half-lives with fast and slow acetylators. Thus, elimination half-lives of either 4.5 or 2.0 $\mathrm{hr}$ were simulated in the model to mimic slow and fast acetylators, respectively. Approximately $200 \mu \mathrm{L}$ of the medium was collected at time zero and every day thereafter until the end of the experiment. The number of viable bacteria in each sample was 
Table 3-1. Experimental Plan with Initial Concentrations of Isoniazid.

\begin{tabular}{ccccc}
\hline Dose Group & $\begin{array}{c}\text { Elimination } \\
\text { Half-life }\end{array}$ & $\begin{array}{c}\text { INH } \\
\text { Dose/day }\end{array}$ & $\begin{array}{c}\text { Number } \\
\text { of Doses }\end{array}$ & $\begin{array}{c}\text { Initial } \\
\text { Concentration } \\
\left(\mathbf{C}_{\mathbf{0}}\right)\end{array}$ \\
\cline { 2 - 5 } Control & $\mathrm{hr}$ & $\mathrm{mg}$ & & $\mathrm{mg} / \mathrm{L}$ \\
\hline Slow & - & - & - & - \\
Acetylators & 4.5 & 25 & 7 & 0.3 \\
& & 100 & 7 & 1.21 \\
Fast & 2.0 & 25 & 7 & 3.64 \\
Acetylators & & 100 & 7 & 0.3 \\
\hline
\end{tabular}

determined by plating serial dilutions of the samples on antibiotic-free Middlebrook $7 \mathrm{H} 11$ agar plates. The plates were incubated for $3-4$ weeks at $37^{\circ} \mathrm{C}$. The antibacterial activity was measured as the reduction in number of viable bacteria, expressed as $\mathrm{CFU} / \mathrm{mL}$. Each experiment was performed in duplicate for each dose level and elimination half-life. No-drug controls were included in the study for comparison to assess the extent of killing by INH. Time-kill curves were constructed based on the time course of $\mathrm{CFU} / \mathrm{mL}$ measurements.

\section{Time-Kill Curve Data Analysis}

The relationship between INH exposure and microbial kill was modeled by using a previously described time-kill curve equation [185],

$$
\frac{d N}{d t}=\left[k_{0} \cdot\left(1-\frac{N}{N_{\max }}\right)-\left(1-e^{-\alpha \cdot t}\right) \times\left(\frac{I_{\max } \cdot C}{I C 50+C}\right)\right] \cdot N
$$

where $\mathrm{N}$ is the $M$. bovis $\mathrm{BCG}$ cell count in $\mathrm{CFU} / \mathrm{mL}, \mathrm{k}_{0}$ is the bacterial net growth rate constant, $\mathrm{N}_{\max }$ is the maximum number of bacteria in the system in CFU/mL, $\mathrm{I}_{\max }$ is the maximum kill rate, $\mathrm{C}$ is the concentration of INH at time $\mathrm{t}$, and IC50 is the concentration at half-maximal kill rate. The logistic growth function, $1-\mathrm{N} / \mathrm{N}_{\max }$ was used to describe the limited growth of bacteria in the absence of antibacterial agents. The delay in bacterial kill rate was modeled using the term $1-\mathrm{e}^{-\alpha \mathrm{t}}$, where $\alpha$ is the delay rate constant. According to the model, the delay rate constant $\alpha$ mathematically modulates $I_{\max }$ over time and determines the duration of time needed for $I_{\max }$ to reach its value. The delay in bacterial kill is likely due to the time necessary to achieve enough intracellular drug exposure to initiate the killing process. The IC50 was modeled as adaptive constant which changes with the exposure and the ratio of the initial cell count to the cell count at time $t$ according to equation, 


$$
I C 50_{A}=I C 50 \times e^{\left(\frac{{\text { Adaptation Constant } \times N_{0}}_{N \times \mathrm{AUC}_{0-24}}}{\mathrm{Ant}}\right)}
$$

where IC $50_{\mathrm{A}}$ is the adaptive IC50, $\mathrm{N}_{0}$ is the number of bacterial cells at time zero and IC50 is the baseline IC50. The adaptive IC50 explains the change in IC50 due to an increased drug insensitive cell population over the course of treatment, such as the frequently observed latency for Mycobacterium species [212, 216-218]. All time-kill data were analyzed simultaneously by nonlinear mixed effects modeling (NONMEM v.6.2, Icon, Ellicott City, MD). PK parameters were fixed in the PK/PD model and set equal to their selected values $(\mathrm{V}=55 \mathrm{~mL}$, where $\mathrm{V}$ is volume of the medium in the system, halflife, $\mathrm{t}_{1 / 2}=4.5$ and $2.0 \mathrm{hr}$ for slow and fast acetylators respectively). The first-order conditional estimation method within NONMEM was used to analyze the generated timekill curves. The measured data and model predictions were log transformed and the random residual variability was modeled using an additive error term. The between experiment variability in the parameter $\alpha$ was modeled as exponential error. Model performance was evaluated using goodness-of-fit plots, including observed vs. predicted, weighted residuals vs. time and weighted residuals vs. predictions plots.

For identification of the most appropriate empirical PK/PD index associated with the microbial kill of INH, cell counts on days 2, 3 and 4 were analyzed using an inhibitory $\mathrm{E}_{\max }$ model [213] expressed as,

$$
E=E_{\text {control }}-\left(\frac{E_{\max } \cdot \mathrm{PKPD}}{E C 50+\mathrm{PKPD}}\right)
$$

where, $\mathrm{E}$ is the observed M. bovis $\mathrm{BCG}$ cell counts in $\log 10 \mathrm{CFU} / \mathrm{mL}, \mathrm{E}_{\text {control }}$ is the $\mathrm{BCG}$ cell count in the control experiment, and $\mathrm{E}_{\max }$ is the maximal antimicrobial effect in $\log 10$ $\mathrm{CFU} / \mathrm{mL}$. EC50 is the value of the PK/PD index that produces half-maximal antimicrobial effect and PKPD is one of the empirical PK/PD indices frequently used in infectious disease pharmacotherapy, $\mathrm{AUC}_{0-24} / \mathrm{MIC}, \mathrm{T}>\mathrm{MIC}$ or $\mathrm{C}_{\max } / \mathrm{MIC}$. The PK/PD index that best characterized the effect of of INH on M. bovis was selected based on goodness-of-fit criteria (e.g., coefficient of determination values), residual analysis and visual inspection.

\section{Results}

Mycobacterium bovis BCG was successfully cultured in our novel in vitro $\mathrm{PK} / \mathrm{PD}$ model for 10 days. The growth rate constant of $M$. bovis BCG in the absence any antibacterial compound was found to be $0.0464 \mathrm{hr}^{-1}$ and the corresponding doubling time/generation time was calculated as 14.9 hours. This doubling time of $M$. bovis BCG is similar to that observed in vitro and comparable to the published range (13-20 hours) [219]. Administration of INH dosing regimens to logarithmically growing cultures of $\mathrm{BCG}$ in the in vitro model resulted in reduced cell counts when compared to the control experiment (Figure 3-3, panels A and B). The initial kill rate during the first two days of 
A)

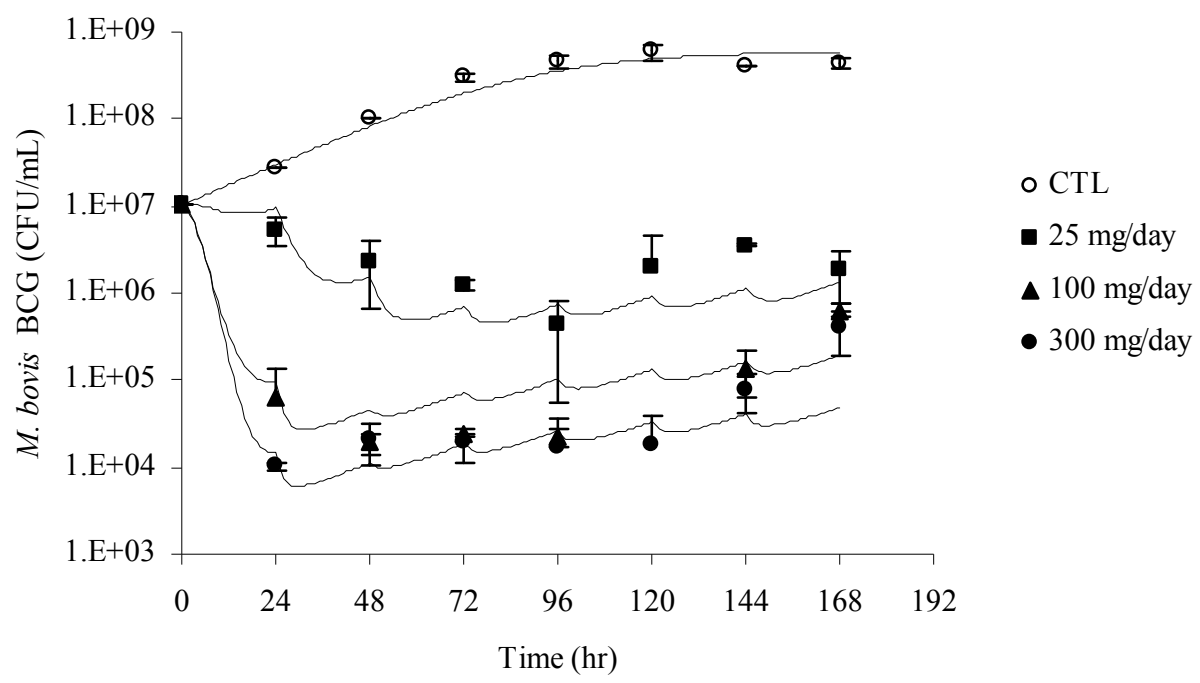

B)

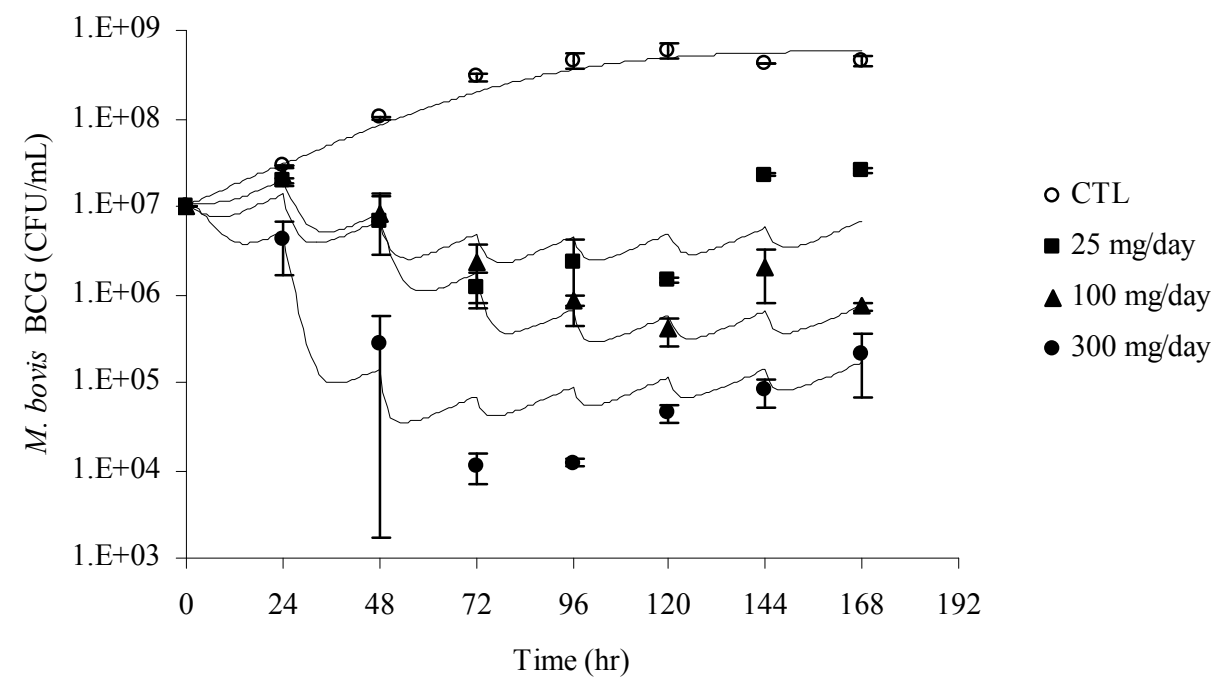

Figure 3-3. Plots Showing Measured (Mean \pm SD) and Model Predicted (Lines) $M$. bovis BCG CFU/mL over Time for the PK/PD Model. Panel A shows kill curves for slow acetylators of isoniazid, panel B for fast acetylators. Symbols for dose groups: Control experiment, $\circ ; 25 \mathrm{mg} /$ day, $\mathbf{\square} ; 00 \mathrm{mg} / \mathrm{day}, \boldsymbol{\Lambda}$; and $300 \mathrm{mg} /$ day, $\bullet$. Lines represent individual predictions based on the PK/PD Model. 
therapy in slow acetylators was $0.32,1.36$, and $1.35 \log 10(\mathrm{CFU} / \mathrm{mL}) /$ day for the 25,100 and $300 \mathrm{mg}$ /day dosing regimens, respectively. Similarly, the initial kill rate for the fast acetylators was calculated as $0.09,0.05$ and $0.78 \log 10(\mathrm{CFU} / \mathrm{mL}) /$ day for the 25,100 and $300 \mathrm{mg}$ /day dosing regimens respectively. The initial kill rate of $\mathrm{INH}$, analogous to in vivo early bactericidal activity (EBA) was concentration- and thus dose-dependent. As concentrations in simulated slow acetylators were higher than in fast acetylators, the INH mediated kill effect was also more pronounced in slow acetylators compared to fast acetylators at each dose level (Figure 3-3, panels A and B). The bactericidal activity appeared with a delay of approximately 24 hours in the fast acetylator dose groups compared to the corresponding dose groups in slow acetylators (Figure 3-3, panels A and B). The INH mediated killing of bacteria ceased after three days of therapy and re-growth of bacteria appeared in both slow and fast acetylator dose groups (Figure 3-3, panels A and B).

Different mathematical models were evaluated for describing the $M$. bovis $\mathrm{BCG}$ time-kill data generated with the in vitro PK/PD model [183, 220-225]. An adaptive IC50 kill curve model was selected to describe the data. Graphical representations showing observed and predicted BCG cell counts at all the tested dosing regimens in slow and fast acetylators are displayed in the panels A and B of Figure 3-3. The corresponding parameter estimates were listed in the Table 3-2. The maximum kill rate $\left(\mathrm{I}_{\max }\right)$ was calculated as $0.852 \mathrm{hr}^{-1}$. The concentration of INH that produces the half-maximal kill rate, IC 50 , was estimated as $0.244 \mathrm{mg} / \mathrm{L}$. The adaptive IC 50, IC $50_{\mathrm{A}}$, was correspondingly ranging from 0.244 to $15.4 \mathrm{mg} / \mathrm{L}$. For comparison, the MIC for INH determined with the classic dilution approach was found to be $0.05 \mathrm{mg} / \mathrm{L}$. The goodness-of-fit plots presented in Figure 3-4 and Figure 3-5 indicate that the selected mathematical time-kill curve model (Eq. 3-1) is able to describe the data well (Figures 3-4 and 3-5).

Examination of the relationship between empirical PK/PD indices and microbial kill for day 3 of the treatment revealed that the $\mathrm{PK} / \mathrm{PD}$ index $\mathrm{AUC}_{0-24} / \mathrm{MIC}$ was the most explanatory of the INH mediated microbial kill (Figure 3-6). The relationships between microbial kill and the other PK/PD indices, $\mathrm{C}_{\max } / \mathrm{MIC}$ and $\mathrm{T}>\mathrm{MIC}$ are also shown in

Table 3-2. Parameter Estimates of Isoniazid PK/PD Model.

\begin{tabular}{lcc}
\hline Parameter & Units & Estimate (\% CV) \\
\hline $\mathrm{k}_{0}$ & $\mathrm{hr}^{-1}$ & $0.0464(61.6)$ \\
IC50 & $\mathrm{mg} / \mathrm{L}$ & $0.244(271)$ \\
Adaptation & $\mathrm{L} / \mathrm{mg} \cdot \mathrm{hr}$ & $0.00224(36.5)$ \\
Constant & $\mathrm{hr}^{-1}$ & $0.852(87.6)$ \\
$\mathrm{I}_{\max }$ & $\mathrm{hr}^{-1}$ & $0.088(85)$ \\
$\alpha$ & &
\end{tabular}

Between Experiment Variability (\%CV) in $\alpha \quad 95.02$ (64.2)

Residual Variability (\%CV) $\quad 81.3(27.1)$ 
A)

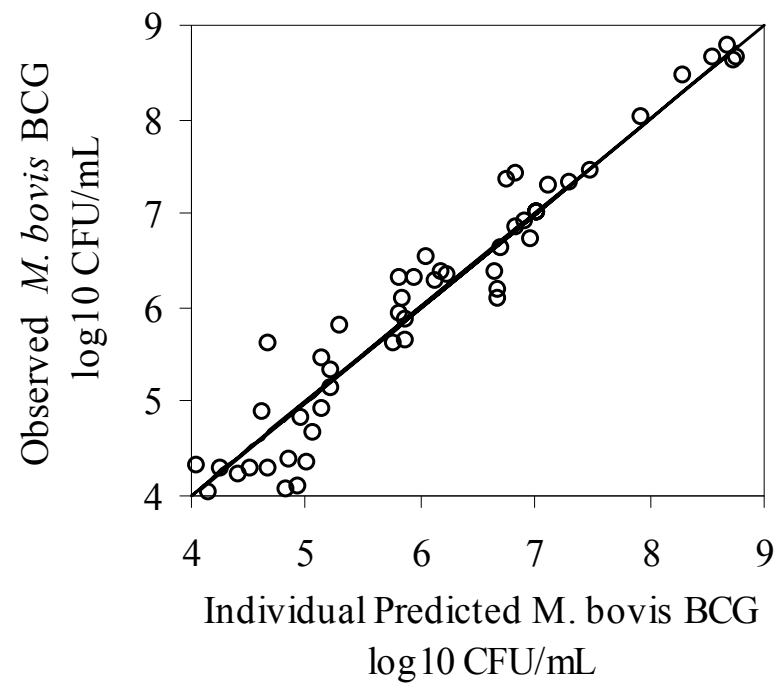

B)

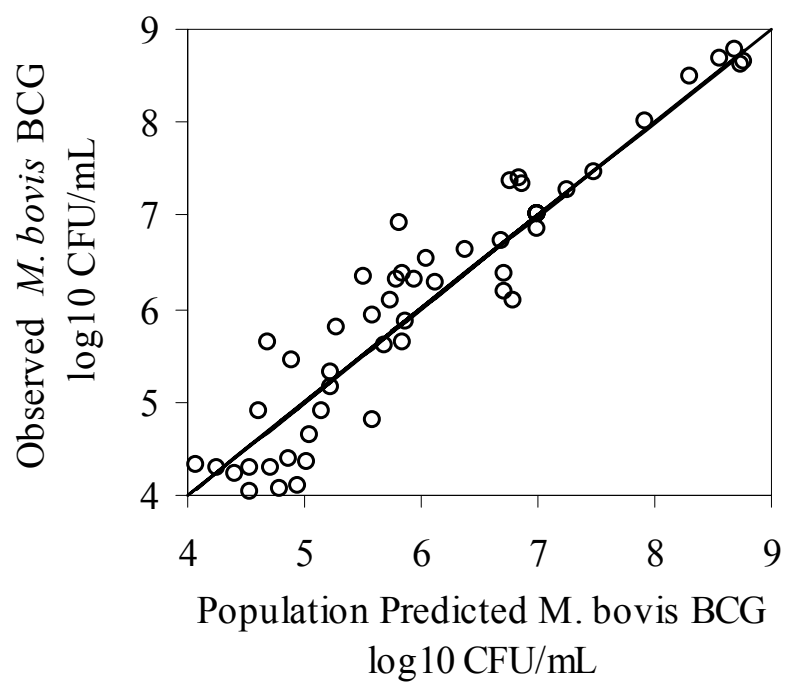

Figure 3-4. Goodness-of-Fit Plots for the INH PK/PD Model - Observed versus Predicted Values. Panel A shows observed vs. individual predicted values $(\log 10$ $\mathrm{CFU} / \mathrm{mL})$ and panel B shows observed vs. population predicted values $(\log 10 \mathrm{CFU} / \mathrm{mL})$. 
A)

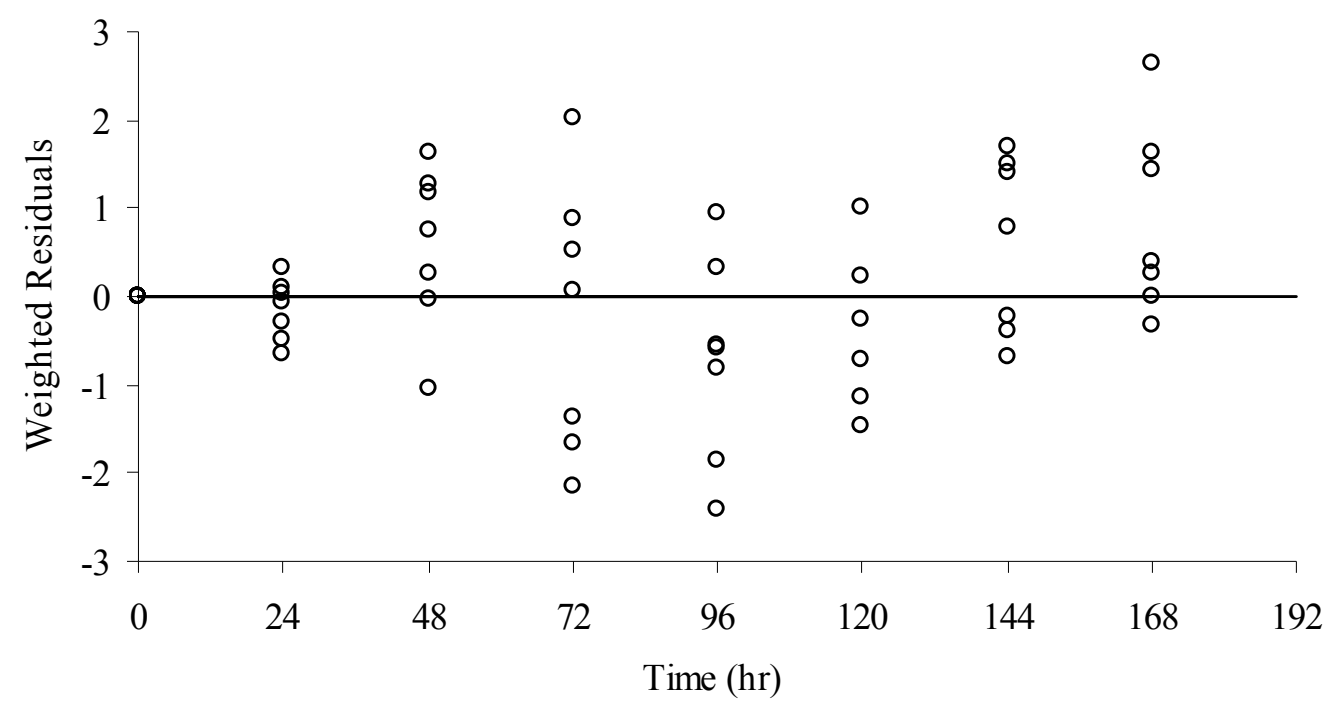

B)

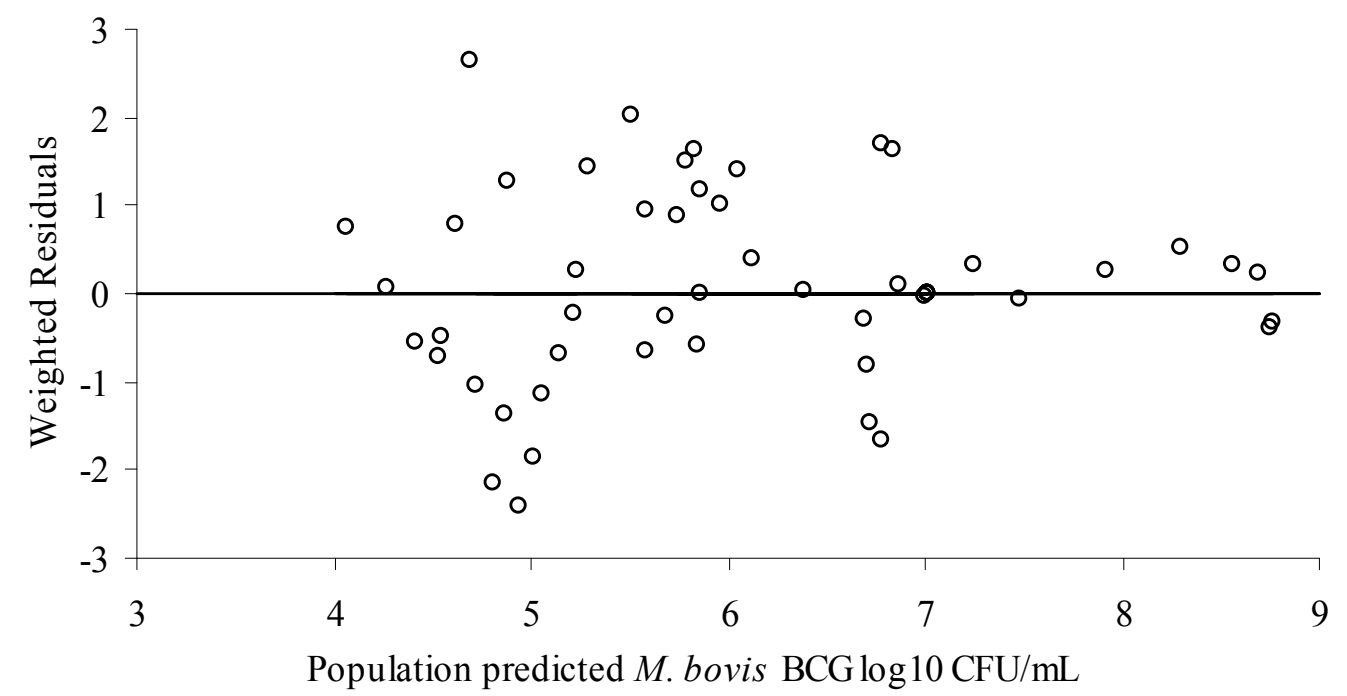

Figure 3-5. Goodness-of-Fit Plots for the INH PK/PD Model - Weighted Residuals. Panel A shows weighted residuals vs. time (hr) and panel B shows weighted residuals vs. population predicted values $(\log 10 \mathrm{CFU} / \mathrm{mL})$. 
A)

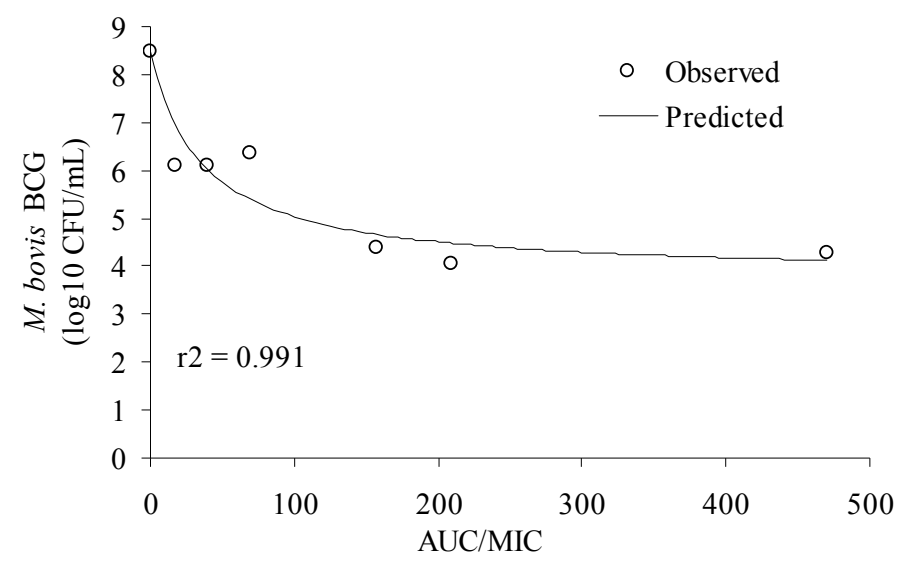

B)

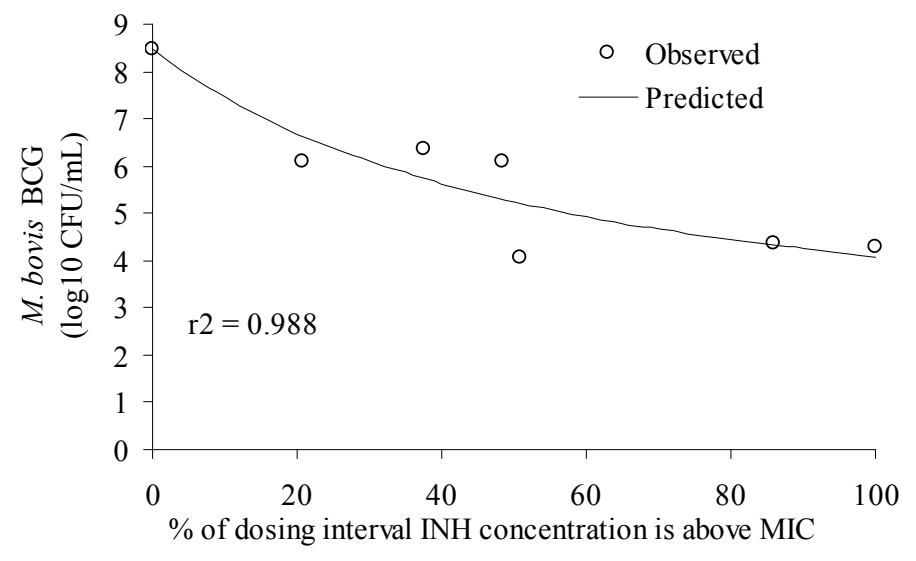

C)

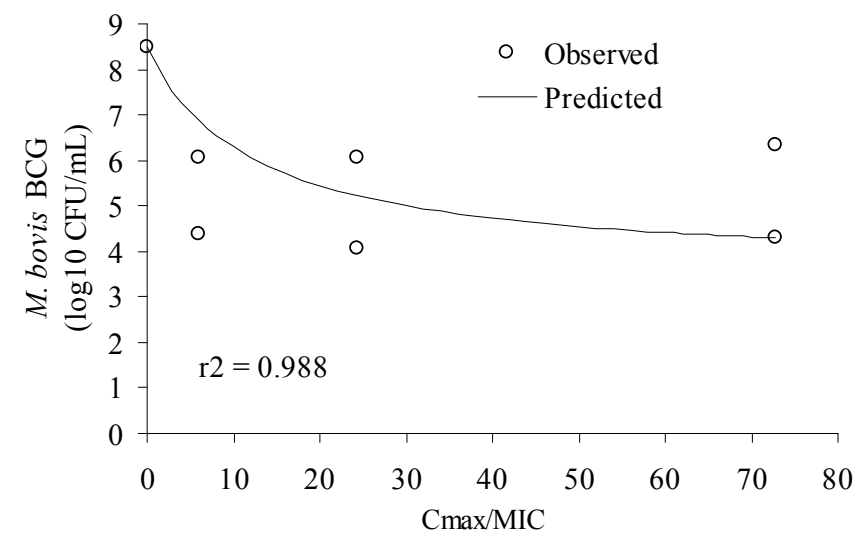

Figure 3-6. Relationship between PK/PD Indices and Microbial Kill on Day 3 after Treatment. Panels A, B, and C show $\mathrm{AUC} / \mathrm{MIC}, \mathrm{T}>\mathrm{MIC}$, and $\mathrm{C}_{\max } / \mathrm{MIC}$, respectively. 
Figure 3-6. Similar results were observed for day 2 and day 4 data (results not shown). The parameter estimates from the empirical PK/PD analysis were given in Table 3-3.

\section{Discussion}

Identification of pharmacodynamic parameters has become an integral part of the development process of novel antibiotics [176]. Characterization of pharmacodynamic parameters in the early stages of development enables the design of optimal dosage regimens for animal and human studies and allows for better predictions of the success/failure of an antibiotic development program [206, 208]. In vitro $\mathrm{PK} / \mathrm{PD}$ models are used widely for the determination of pharmacodynamic parameters of antibiotics $[184,220,223,224,226-228]$ due to a variety of advantages. One of the advantages of in vitro models is that human and animal pharmacokinetic profiles can easily be simulated. In addition, the lack of immune system in these in vitro experiments allows for precise characterization of bacteria-drug interactions. Further, the in vitro models are better suited over animal models to test and identify the time- or concentration-dependant killing nature of antibacterial drugs [201]. The novel in vitro PK/PD model described in this manuscript belongs to the dilution model category with a filter membrane to prevent bacterial dilution. It is easily constructed, inexpensive, prevents dilution of the bacteria without membrane pore blockage and can be used for slow growing organisms as shown with the time-kill analysis using $M$. bovis BCG. Compared to the previously reported models of similar nature [204, 205], the model described in this paper has several advantages: 1) It is a completely closed system and is safe to work with pathogenic strains, 2) the model can be used to work with slow and fast growth rate organisms, 3) the temperature in the flask can be controlled precisely with a water jacket and, 4) assembling and disassembling of the components of the model is simple and straight forward, allowing for easy study conduct and equipment sterilization.

Slow growth rate microorganisms such as $M$. bovis BCG pose many challenges for in vitro culturing. Much longer periods, usually more than a week are required to assess the efficacy of an antibacterial agent against these slow growing organisms when compared to fast growing organisms. Consequently, integrity of the filter membrane is crucial for allowing media exchange but preventing outflow of bacteria throughout the duration of experiment. In addition, the in vitro PK/PD model should not hinder natural growth of bacteria in the absence of antibacterial agents. The concordance between the

Table 3-3. Empirical PK/PD Indices.

\begin{tabular}{ccccc}
\hline Parameter & Units & AUC $_{\mathbf{0 - 2 4}} / \mathbf{M I C}$ & $\mathbf{T}>\mathbf{M I C}(\%)$ & $\mathbf{C}_{\mathbf{m a x}} / \mathbf{M I C}$ \\
\hline $\mathbf{E}_{\mathbf{m a x}}$ & log10 CFU/mL & 4.69 & 6.94 & 4.90 \\
$\mathbf{E C 5 0}$ & variable dependent & 36.01 & 57.33 & 12.33 \\
$\mathbf{R}^{\mathbf{2}}$ & on index & 0.991 & 0.988 & 0.988 \\
\hline
\end{tabular}


observed doubling time for $M$. bovis BCG and the literature reported values suggests that the in vitro $\mathrm{PK} / \mathrm{PD}$ model described in this report is not interfering with the normal growth of $M$. bovis BCG.

INH is one of the first line agents used in the multi drug combination therapy for TB that kills actively dividing bacteria. Early bactericidal activity (EBA) of the combination drug therapy for TB is derived mainly from INH activity on the logarithmically growing bacteria $[229,230]$. EBA is defined as the rate of fall of colony forming units $(\mathrm{CFU} / \mathrm{mL})$ in the sputum of patients during the first two days of drug treatment and is expressed as $\log 10(\mathrm{CFU} / \mathrm{mL}) /$ day. PK/PD models based on in vitro time-kill curves have been used to assess the efficacy of antimicrobial agents. However, reports about the use of such models for anti-tuberculosis drugs are scarce. Only a few recently reported in vitro models characterized dynamic time-kill curves of the antituberculosis agents INH, rifampicin and moxifloxacin [212-215]. From the results of the time-kill experiments performed in this study, it can be seen that the kill rate of bacteria is different at identical dosing regimens in the slow and fast acetylator groups of INH. An initial delay in killing was observed at all the dosing regimens studied in the fast acetylator groups in contrast to the slow acetylator dose groups (Figure 3-3, panels A and B). In an in vitro study with ${ }^{14} \mathrm{C}$-labeled $\mathrm{INH}$, it was shown that it takes more than 2 hours for complete intracellular uptake of INH when incubated with $M$. bovis BCG [231]. Since INH is eliminated with a half-life of 2 hours in fast acetylators the observed delay in killing could be due to the rapid removal of drug from the medium in this group compared to slow acetylators (half-life $4.5 \mathrm{hr}$ ). Similarly, the rapid removal of drug in the fast acetylators is likely the reason for the observed differences in the initial kill rates of bacteria among the identical dose groups in both slow and fast acetylators. The exposuredependant kill pattern for mycobacteria under INH therapy has been characterized in previously reported in vitro and in vivo animal studies [167, 215]. The initial rapid kill rate followed by a slow kill rate along with the re-growth of bacteria observed in our study is remarkably similar to that observed in an in vitro study by Gumbo et al., using M. tuberculosis H37Ra [215]. Similar results were found in several other in vitro drug susceptibility assays. It was hypothesized that the development of transient resistance to INH is responsible for the cessation of INH bactericidal activity [217, 232].

We used a semi-mechanistic PK/PD model to describe the rate of killing of bacteria over time at different dosing regimens. The principle behind the analysis is that the change in CFUs over time is governed by the difference in the rate of growth and the rate of killing with INH stimulating the rate of microbial kill. The limiting growth of bacteria in the absence of INH was described by a logistic function, $1-N / N_{\max }$ where, $N$ is the number of CFU/mL at time $\mathrm{t}$ and $N_{\max }$ is the maximum number of bacteria $(\mathrm{CFU} / \mathrm{mL})$ in the system which corrects for the limitations of space and nutrients that are inherent to in vitro systems $[185,220,225]$. A correction factor $\left(1-\mathrm{e}^{-\alpha t}\right)$ was incorporated to describe the delay in kill effect after the addition of INH in the fast acetylator dose groups [185]. Since the numbers of INH insensitive populations of bacteria due to latency or resistance increased over the course of treatment, the selected PK/PD model needed to be able to describe the re-growth of bacteria. Various approaches have been reported for modeling drug resistance and/or growth of drug insensitive populations of bacteria [183, 213, 220- 
225]. Here, we used an adaptive IC50 model to describe the increase in apparent IC50 due to growth of drug insensitive populations of bacteria. The increase in IC50 was inversely related to the INH exposure and the ratio of cell counts to the initial cell numbers (Eq. 3-2). This means that low exposure to INH results in increased development of insensitivity which is manifested as an increase in apparent IC50. This model was able to describe the observed re-growth of bacteria after several days of drug exposure. The IC50 was estimated as $0.244 \mathrm{mg} / \mathrm{L}$ and could increase over the course of treatment up to $15.4 \mathrm{mg} / \mathrm{L}$. The stationary concentration of INH which is defined as the concentration at which the rate of bacterial killing equals growth was calculated using IC50 of INH as described by Mouton et al.[233]. The calculated stationary concentration $(0.014 \mathrm{mg} / \mathrm{L})$ was much lower than the MIC $(0.05 \mathrm{mg} / \mathrm{L})$, a commonly observed phenomenon for the concentration/exposure dependent killing by antibiotics [233].

In the current study, INH exhibited exposure-dependent antibacterial activity on M. bovis $\mathrm{BCG}$. The empirical $\mathrm{PK} / \mathrm{PD}$ index $\mathrm{AUC}_{0-24} / \mathrm{MIC}$ was found to be well associated with the microbial kill. In contrast, the other tested indices, $\mathrm{C}_{\max } / \mathrm{MIC}$ and $\mathrm{T}>\mathrm{MIC}$, were not correlated well with microbial kill. Not surprisingly, the finding that the INH kill rate is exposure-dependant is in agreement with previously published in vitro and in vivo studies $[167,212,215]$. However, the parameter estimate $\left(\mathrm{AUC}_{0-24} / \mathrm{MIC}\right)$ from a previous study, 61.6 is different from that of the current study, 36.0. The difference is likely due to the difference in the test organisms' susceptibility to INH ( $M$. tuberculosis $\mathrm{H} 37 \mathrm{Ra}$ vs. M. bovis $\mathrm{BCG})$.

The novel in vitro $\mathrm{PK} / \mathrm{PD}$ model and associated $\mathrm{PK} / \mathrm{PD}$ modeling approach described here can be used as a tool to evaluate and predict the bactericidal activities of novel anti-tuberculosis compounds. The underlying principle is to describe the whole time course of the events seen in the bacterial system when exposed to antibiotics. Such models, when combined with other information such as toxicology data, can be used to design improved dosing regimens of antibiotics beyond the empirical approaches. Simulations based on the mechanistically based time-kill models allow for evaluation and comparison of previously tested and hypothetical, untested dosing strategies.

The in vitro time-kill curve approach described for anti-mycobacterial drug activity, however, has some limitations. The current study was performed with an assumption that the plasma concentrations are similar to the effect site concentrations. However, in most of cases in vivo effect site concentrations are difficult to determine and may differ from plasma concentrations dependent on protein binding and tissue uptake. Secondly, the environment for bacterial growth in vitro differs from that in vivo. Thirdly, the drug effect was measured on logarithmically growing bacteria which only describes the EBA of a drug. Thus, the approach described here cannot predict the sterilizing activity of drugs on persister (slowly metabolizing) populations of mycobacteria which are thought of being frequently responsible for relapses under drug therapy. This is one of the major limitations of most in vitro and in vivo models currently available for evaluating drug activity in tuberculosis. 


\section{Conclusion}

In conclusion, we have shown that a newly developed in vitro PK/PD model can be used to determine time-kill curves for slow growing organisms as exemplified by the determination of time-kill curves for dosing regimens of INH against $M$. bovis BCG. We have also shown that the time-kill data of INH against $M$. bovis BCG can be described using an adaptive IC50 model that corrects for increased drug insensitive populations of bacteria during the course of treatment. The newly developed model can be used to determine time-kill curves of novel anti-tuberculosis compounds to aid in their development. Also, the approach can be used to evaluate new dosing regimens of currently used anti-tuberculosis agents. 


\section{CHAPTER 4. PHARMACODYNAMICS OF LEE 1106 AGAINST M. BOVIS BCG IN AN IN VITRO PK/PD MODEL}

\section{Introduction}

In an effort to develop new and more potent therapies to treat TB, a novel class of anti-infectives with high in vitro activity against $M$.tuberculosis, nitrofuranylamides, has recently been characterized by our group [189-192, 234]. The compound Lee 1106 emerged from the pharmacokinetically-guided paradigm described in chapter 2 and possesses the characteristics of a lead compound suitable for further development [235]. Lee 1106 was found to have good in vitro activity against $M$. tuberculosis with an MIC of $0.025 \mathrm{mg} / \mathrm{L}$. Also, Lee 1106 exhibited good metabolic stability with more than $70 \%$ of the parent compound remaining stable after 90 minutes of incubation with co-factor supplemented liver microsomal preparation. This in vitro observation translated into an increased in vivo stability (lower plasma clearance) and a longer elimination half-life of Lee 1106 in rats compared to other compounds in the nitrofuranylamide series. On the other hand, however, the compound exhibited a poor oral bioavailability of $4.6 \%$, most likely secondary to poor aqueous solubility [235]. With the recent advances in solubilization technology, the compound's apparent aqueous solubility can be enhanced using solubilizing excipients [200], and this approach is expected to increase its oral bioavailability. The stability of Lee 1106 in liver microsomal preparations indicates only limited, if any, involvement of the cytochrome P450 (CYP450) enzyme system in the clearance of the compound. This absence of major CYP450-mediated metabolism increases the likelihood of compatibility with existing anti HIV medications which are mainly metabolized by the CYP450 enzyme system and are frequently co-administered with anti-tuberculosis agents [188, 236].

In the current chapter we investigated the time course of bacterial kill of Lee 1106 in an in vitro $\mathrm{PK} / \mathrm{PD}$ model previously developed by our group that simulates the fluctuating concentrations in the in vivo pharmacokinetic profile of Lee 1106. The Lee 1106-mediated bacterial kill was characterized at different multiple-dose regimens using M. bovis BCG as a model organism. An integrated PK/PD model was developed based on the generated bacterial time-kill curves that was able to describe the anti-mycobacterial effect of Lee 1106 on M. bovis BCG for all tested dosing regimens. The PK/PD model was subsequently used for numerical simulations to predict the antibacterial effect of Lee 1106 for untested dosing regimens. This approach gained valuable insights into the potential in vivo anti-mycobacterial activity of Lee 1106 and may guide dose selection and study designs for future in vivo evaluations. 


\section{Materials and Methods}

\section{Culture, Media, and Antibiotics}

Mycobacterium bovis BCG was grown in Middlebrook 7H9 broth (Becton Dickinson, Sparks, MD) supplemented with $10 \%$ albumin dextrose complex and $0.1 \%$ $\mathrm{v} / \mathrm{v}$ Tween 80 . The cultures were stored at $-80^{\circ} \mathrm{C}$ in Middlebrook $7 \mathrm{H} 9$ broth. For each experiment, aliquots were thawed and incubated at $37^{\circ} \mathrm{C}$ in Middlebrook $7 \mathrm{H} 9$ broth for 4 days.

The test compound Lee 1106 (1-\{4-[3-(5-nitro-2-furyl)-4, 5-dihydroisoxazol-5-yl] phenyl $\}-4-[4-($ trifluoromethoxy) phenoxy] piperidine) was synthesized in the laboratory of Dr. R.E. Lee at the University of Tennessee Health Science Center, Memphis, TN (Figure 2-1, Chapter 2). A stock solution of Lee 1106 was prepared in DMSO and stored at $-80^{\circ} \mathrm{C}$. Working solutions of the compound were prepared from the stock solution using DMSO.

\section{Minimum Inhibitory Concentration of Lee 1106}

The MIC of Lee 1106 was determined using the microbroth dilution method according to the CLSI guidelines [73] and was read by visual inspection. Two fold serial dilutions of Lee 1106 in $100 \mu \mathrm{L}$ of the Middlebrook 7H9 broth medium were prepared in round-bottomed micro liter plates (Nunc, Sigma-Aldrich, St. Louis, MO). An equivalent volume $(100 \mu \mathrm{L})$ of broth containing $\sim 10^{5} \mathrm{CFU} / \mathrm{mL}$ bacteria was added to each well to give final concentration of Lee 1106 starting at $200 \mathrm{mg} / \mathrm{L}$ and the plates were incubated aerobically at $37^{\circ} \mathrm{C}$ for 7 days. The MIC was recorded as the lowest concentration of the compound that prevented visible growth.

\section{In vitro PK/PD Model Design}

A description of the in vitro PK/PD model design is presented in Chapter 3 of this dissertation.

\section{Time-Kill Curves of Lee 1106}

The test organism $M$. bovis BCG was used to determine the time-kill curves. $M$. bovis BCG was grown to early logarithmic growth phase in Middlebrook7H9 medium. The logarithmically growing culture was then used to inoculate the in vitro model. The final density of the culture in the in vitro system was $\sim 10^{6} \mathrm{CFU} / \mathrm{mL}$. The medium was drawn from the flask via the combination of a $5 \mu \mathrm{m}$ and a $0.22 \mu \mathrm{m}$ filter at a predetermined flow rate that mimicked the in vivo elimination half-life of Lee 1106.

Fresh sterile medium was pumped into the flask at the same rate through one side arm to 
keep the volume of the medium at the initial level. The addition of Lee 1106 doses was started after the culture had reached the logarithmic growth phase which was determined by the optical density of the culture. Lee 1106 doses were added daily for 7 days to simulate Lee 1106 plasma concentration-time profiles in rats treated with $0.1,0.3,1,3$ and $10 \mathrm{mg} / \mathrm{kg}$ /day given once daily (Table 4-1). An elimination half-life of $10.3 \mathrm{hr}$ was simulated in the in vitro model. The elimination half-life 10.3 hours was adapted from the intravenous pharmacokinetic data of Lee 1106 in rats [235]. Approximately $200 \mu \mathrm{L}$ of the sample was collected at time zero and every day thereafter until the end of the experiment. The number of viable bacteria in each sample was determined by plating serial dilutions of the samples on antibiotic-free Middlebrook 7H11 agar plates. The bacterial cells were washed twice to remove the residual compound before plating. The plates were incubated for $3-4$ weeks at $37^{\circ} \mathrm{C}$. The antibacterial activity was measured as the reduction in number of viable bacteria, expressed as CFU $/ \mathrm{mL}$. No-drug control was included in the study to assess the extent of killing by Lee 1106. The time-kill curves were constructed based on the time course of $\mathrm{CFU} / \mathrm{mL}$ measurements.

\section{Time-Kill Curve Data Analysis}

The relationship between Lee 1106 exposure and microbial kill was modeled by using a previously described time-kill curve equation [185],

$$
\frac{d N}{d t}=\left[k_{0} \times\left(1-\frac{N}{N_{\max }}\right)-\left(\frac{I_{\max } * C}{I C 50+C}\right)\right] \times N
$$

where $\mathrm{N}$ is the $M$. bovis $\mathrm{BCG}$ cell count in $\mathrm{CFU} / \mathrm{mL}, \mathrm{k}_{0}$ is the bacterial net growth rate constant, $\mathrm{N}_{\max }$ is the maximum number of bacteria in the system in CFU/mL, $I_{\max }$ is the maximum kill rate, $\mathrm{C}$ is the concentration of Lee 1106 at time $\mathrm{t}$, and IC50 is the concentration at half-maximal kill rate. The logistic growth function, $1-\mathrm{N} / \mathrm{N}_{\max }$ was used to describe the limited growth of bacteria in the absence of antibacterial agents. The IC50 was modeled as adaptive constant which changes with the exposure and the ratio of the initial cell count to the cell count at time $t$ according to equation,

Table 4-1. Experimental Plan with Initial Concentrations of Lee 1106.

\begin{tabular}{ccccc}
\hline $\begin{array}{c}\text { Dose } \\
\text { Group }\end{array}$ & $\begin{array}{c}\text { Elimination } \\
\text { Half-life }\end{array}$ & $\begin{array}{c}\text { Lee 1106 } \\
\text { Dose/day }\end{array}$ & $\begin{array}{c}\text { Number of } \\
\text { Doses }\end{array}$ & $\begin{array}{c}\text { Initial Conc. } \\
\left(\mathbf{C}_{\mathbf{0}}\right)\end{array}$ \\
\cline { 2 - 5 } Control & $\mathrm{hr}$ & $\mathrm{mg} / \mathrm{kg}$ & & $\mathrm{mg} / \mathrm{L}$ \\
& - & - & - & - \\
& & 0.1 & 7 & 0.115 \\
Treatment & 10.3 & 0.3 & 7 & 0.345 \\
& & 1 & 7 & 1.15 \\
& & 3 & 7 & 3.45 \\
& & 10 & 7 & 11.5 \\
\hline
\end{tabular}




$$
I C 50_{A}=I C 50 \times e^{\left(\frac{\text { Adaptation Constant } \times N_{0}}{N \times \mathrm{AUC}_{0-24}}\right) * t}
$$

where IC50 $\mathrm{A}$ is the adaptive IC50, $\mathrm{N}_{0}$ is the number of bacterial cells at time zero and IC50 is the baseline IC50. The adaptive IC50 explains the change in IC50 due to an increased drug insensitive cell population over the course of treatment, such as the frequently observed latency for Mycobacterium species [212, 216-218]. All time-kill data were analyzed simultaneously by nonlinear mixed effects modeling (NONMEM v.6.2, Icon, Ellicott City, MD). PK parameters were fixed in the PK/PD model and set equal to their selected values $(\mathrm{V}=55 \mathrm{~mL}$, where $\mathrm{V}$ is volume of the media in the system, half-life, $\mathrm{t}_{1 / 2}=10.3 \mathrm{hr}$ ) [235]. The first-order conditional estimation method in NONMEM was used to analyze the generated time-kill curves. The data and model predictions were log transformed and the random residual variability was modeled using an additive error term. The between experiment variability in the parameters IC50 and adaptation constant was modeled as exponential error. Model performance was analyzed using goodness-offit plots, including observed vs. predicted, weighted residuals vs. time and weighted residuals vs. predictions plots. In addition, a visual predictive check was performed to evaluate the model. One thousand datasets were simulated from the PK/PD model and $90 \%$ prediction intervals from the simulated datasets were overlaid on the observed data.

For identification of the most appropriate empirical PK/PD index associated with the microbial kill, cell counts on days 2, 3 and 4 were analyzed using an inhibitory sigmoid $\mathrm{E}_{\max }$ model [213] expressed as,

$$
E=E_{\text {control }}-\left(\frac{E_{\max } * \mathrm{PKPD}^{\mathrm{H}}}{E C 50^{\mathrm{H}}+\mathrm{PKPD}^{\mathrm{H}}}\right)
$$

where, $\mathrm{E}$ is the observed M. bovis $\mathrm{BCG} \log 10 \mathrm{CFU} / \mathrm{mL}, \mathrm{E}_{\text {control }}$ is the $\mathrm{BCG}$ cell count in the control experiment, $\mathrm{E}_{\max }$ is the maximal antimicrobial effect in $\log 10 \mathrm{CFU} / \mathrm{mL}, \mathrm{EC} 50$ is the value of $\mathrm{PK} / \mathrm{PD}$ index that produces half-maximal antimicrobial effect, $\mathrm{H}$ is the hill coefficient and PKPD is one of the empirical $\mathrm{PK} / \mathrm{PD}$ indices frequently used in infectious diseases pharmacotherapy, $\mathrm{AUC}_{0-24} / \mathrm{MIC}$ and $\mathrm{C}_{\max } / \mathrm{MIC}$. The $\mathrm{PK} / \mathrm{PD}$ index 'time above minimum inhibitory concentration' $\mathrm{T}>\mathrm{MIC}$ was not estimated because the concentrations of Lee 1106 were above MIC 100\% of the time in the steady-state dosing intervals at all dose levels except $0.1 \mathrm{mg} / \mathrm{kg}$. The PK/PD index that best characterized the effect of Lee 1106 on M. bovis was selected based on goodness-of-fit criteria (e.g., coefficient of determination values), residual analysis and the visual inspection.

\section{Numerical Simulations}

Numerical simulation experiments were performed based on the PK/PD model to predict the bactericidal effect of Lee 1106 on M. bovis BCG at untested dosing regimens in mice, the species frequently used for in vivo infection model of mycobacteria. The pharmacokinetics of Lee 1106 in mouse were extrapolated from the rat pharmacokinetics 
by using allometric scaling. Dosing regimens of Lee 1106 with twice daily, once daily, twice weekly and once weekly administration (dosing intervals 12, 24, 72 and $168 \mathrm{hr}$, respectively) were simulated using the PK/PD model developed from the experimental time-kill data collected at once daily dosing regimens. One thousand datasets were simulated for each dosing regimen, and median and $90 \%$ prediction intervals of $M$. bovis BCG cell counts were calculated and plotted against time.

\section{Results}

The growth rate constant of $M$. bovis $\mathrm{BCG}$ in the in vitro $\mathrm{PK} / \mathrm{PD}$ model was found to be $0.0568 \mathrm{hr}^{-1}$ and the corresponding doubling time/generation time was calculated as 12.2 hours. This doubling time of $M$. bovis BCG is similar to that observed in the timekill curves of isoniazid, $14.9 \mathrm{hr}$ (Chapter 3) and comparable to the published range (13-20 hours) [219]. Administration of Lee 1106 dosing regimens to logarithmically growing cultures of $M$. bovis $\mathrm{BCG}$ in the in vitro $\mathrm{PK} / \mathrm{PD}$ model resulted in reduced cell counts in a dose-dependent manner (Figure 4-1). The initial kill rate during the first two days of therapy was $-0.35,0.57,0.99,2.12$ and $1.67 \log 10(\mathrm{CFU} / \mathrm{mL}) /$ day for the $0.1,0.3,1,3$ and $10 \mathrm{mg} / \mathrm{kg} /$ day dosing regimens, respectively (Figure 4-2). The observed initial kill rates were dose-dependent. No killing was observed with the $0.1 \mathrm{mg} / \mathrm{kg}$ dosing regimen and the highest killing was observed with the $3 \mathrm{mg} / \mathrm{kg} /$ day regimen. The killing effect reached a plateau at the $3 \mathrm{mg} / \mathrm{kg}$ dosing regimen. Further increases in dose up to 10 $\mathrm{mg} / \mathrm{kg}$ did not produce any additional increase in the bacterial kill rate. No delay was observed between the bactericidal effect of Lee 1106 and the drug exposure at all studied dosing regimens.

The time-kill data was analyzed using an adaptive IC50 kill curve model based on Eq. 4-1. The adaptive IC50 function was implemented based on Eq. 4-2. Graphical representations showing observed and predicted BCG cell counts at all the tested dosing regimens are displayed in Figure 4-1. The corresponding parameter estimates are listed in Table 4-2. The maximum kill rate $\left(\mathrm{I}_{\max }\right)$ was calculated as $0.338 \mathrm{hr}^{-1}$. The concentration of Lee 1106 that produces the half-maximal kill rate, IC50, was estimated as $0.55 \mathrm{mg} / \mathrm{L}$. The adaptive IC50, IC $50_{\mathrm{A}}$, was correspondingly ranging from $0.55 \mathrm{mg} / \mathrm{L}$ at early to 31.4 $\mathrm{mg} / \mathrm{L}$ at late time points. For comparison, the MIC for Lee 1106 determined with the classic dilution approach was found to be $0.025 \mathrm{mg} / \mathrm{L}$. The goodness-of-fit plots presented in Figure 4-3 and Figure 4-4 indicate that the PK/PD model (Eq. 4-1) is able to describe the data well (Figure 4-1). This notion is supported by a visual predictive check that related the $90 \%$ prediction intervals to the experimentally obtained data (Figure 4-5, panels A to F).

Examination of the relationship between empirical PK/PD indices and microbial kill for day 3 of the treatment revealed that the PK/PD indices $\mathrm{AUC}_{0-24} / \mathrm{MIC}$ and $\mathrm{C}_{\max } / \mathrm{MIC}$ both were able to describe the Lee 1106 mediated microbial kill (Figure 4-6). Similar results were observed for day 2 and day 4 data (results not shown). The parameter estimates from the empirical PK/PD analysis are listed in Table 4-3. The relationship between microbial kill and the PK/PD index, $\mathrm{T}>\mathrm{MIC}$ could not be determined since the 


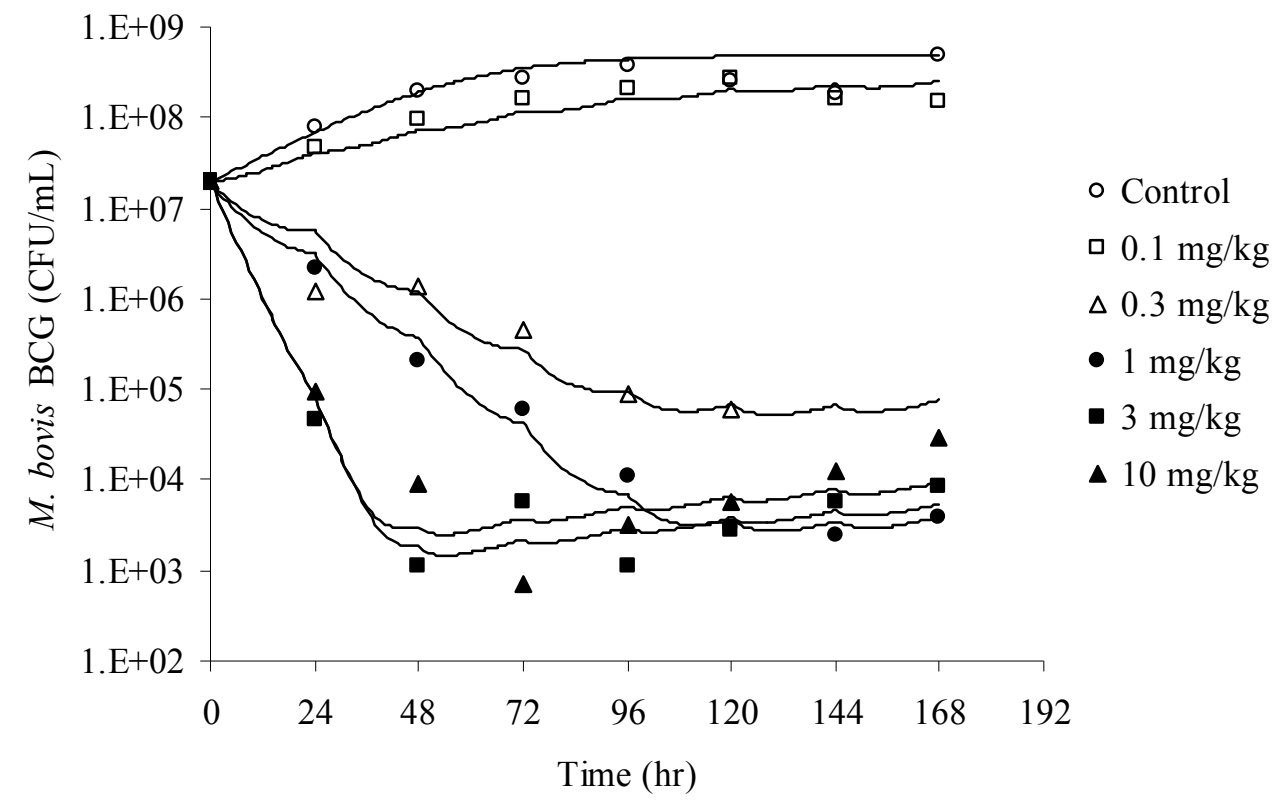

Figure 4-1. Measured (Symbols) and PK/PD Model Predicted (Lines) M. bovis BCG $\mathrm{CFU} / \mathrm{mL}$ over Time for Different Dosing Regimens of Lee 1106. Control experiment, $\circ$; $0.1 \mathrm{mg} / \mathrm{kg} / \mathrm{day}, \square ; 0.3 \mathrm{mg} / \mathrm{kg} / \mathrm{day}, \Delta ; 1 \mathrm{mg} / \mathrm{kg} / \mathrm{day}, \bullet ; 3 \mathrm{mg} / \mathrm{kg} / \mathrm{day}$, $\mathbf{\square}$; and $10 \mathrm{mg} / \mathrm{kg} / \mathrm{day}$, $\boldsymbol{\Delta}$. Lines represent individual predictions based on the PK/PD Model. 


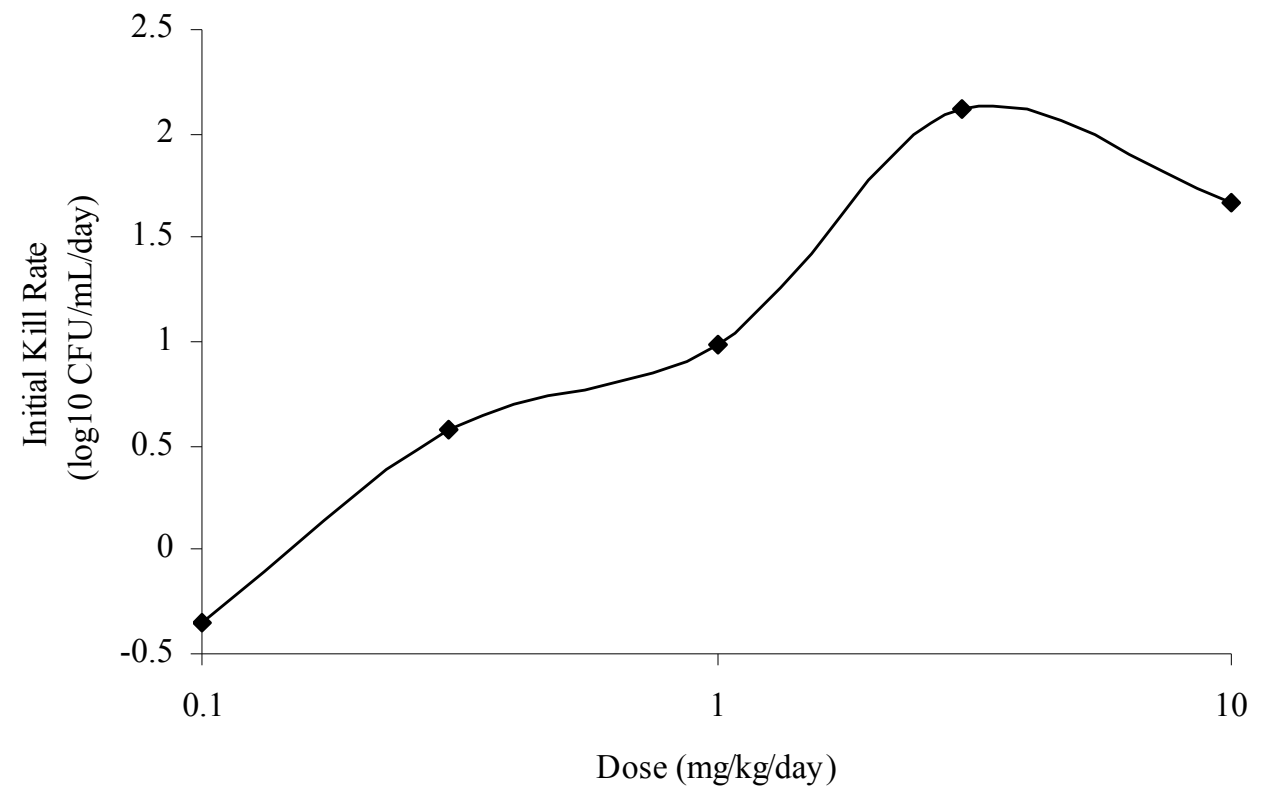

Figure 4-2. Initial Kill Rates of Lee 1106 Plotted against Dose. 
Table 4-2. Parameter Estimates of Lee 1106 PK/PD Model.

\begin{tabular}{lccc}
\hline \multicolumn{1}{c}{ Parameter } & Units & $\begin{array}{c}\text { Estimate } \\
(\% \mathbf{C V})\end{array}$ & $\begin{array}{c}\text { Between Experiment } \\
\text { Variability in \%CV }\end{array}$ \\
\hline $\mathbf{k}_{\mathbf{0}}$ & $\mathrm{hr}^{-1}$ & $0.0568(15.7)$ & $*$ \\
IC50 & $\mathrm{mg} / \mathrm{L}$ & $0.55(35.3)$ & 57.1 \\
Adaptation & $\mathrm{L} / \mathrm{mg} \cdot \mathrm{hr}$ & $0.00016(91.2)$ & 154 \\
Constant & $\mathrm{hr}^{-1}$ & $0.338(13.1)$ & $*$ \\
$\mathbf{I}_{\mathbf{m a x}}$ & $60.9(33.4)$ & \\
\hline Residual Variability (\%CV) & & \\
\hline
\end{tabular}

*No variability estimated for this parameter. 
A)

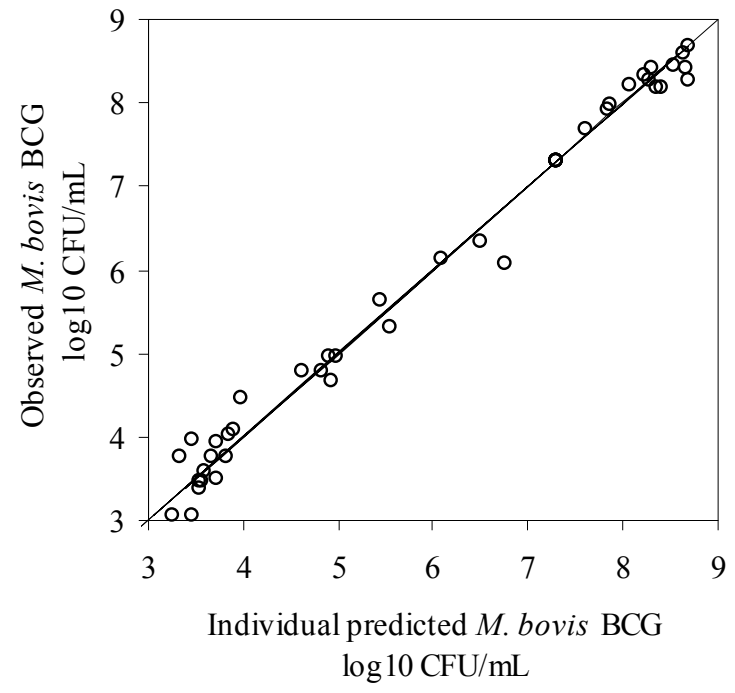

B)

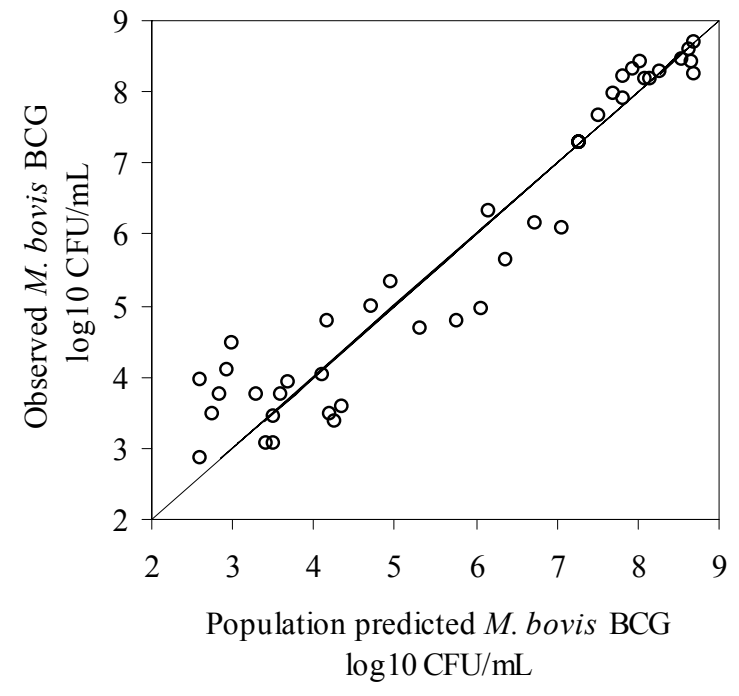

Figure 4-3. Goodness-of-Fit Plots for the Lee 1106 PK/PD Model - Observed versus Predicted Values. Panel A shows observed vs. individual predicted values $(\log 10$ $\mathrm{CFU} / \mathrm{mL})$ and panel B shows observed vs. population predicted values $(\log 10 \mathrm{CFU} / \mathrm{mL})$. 
A)

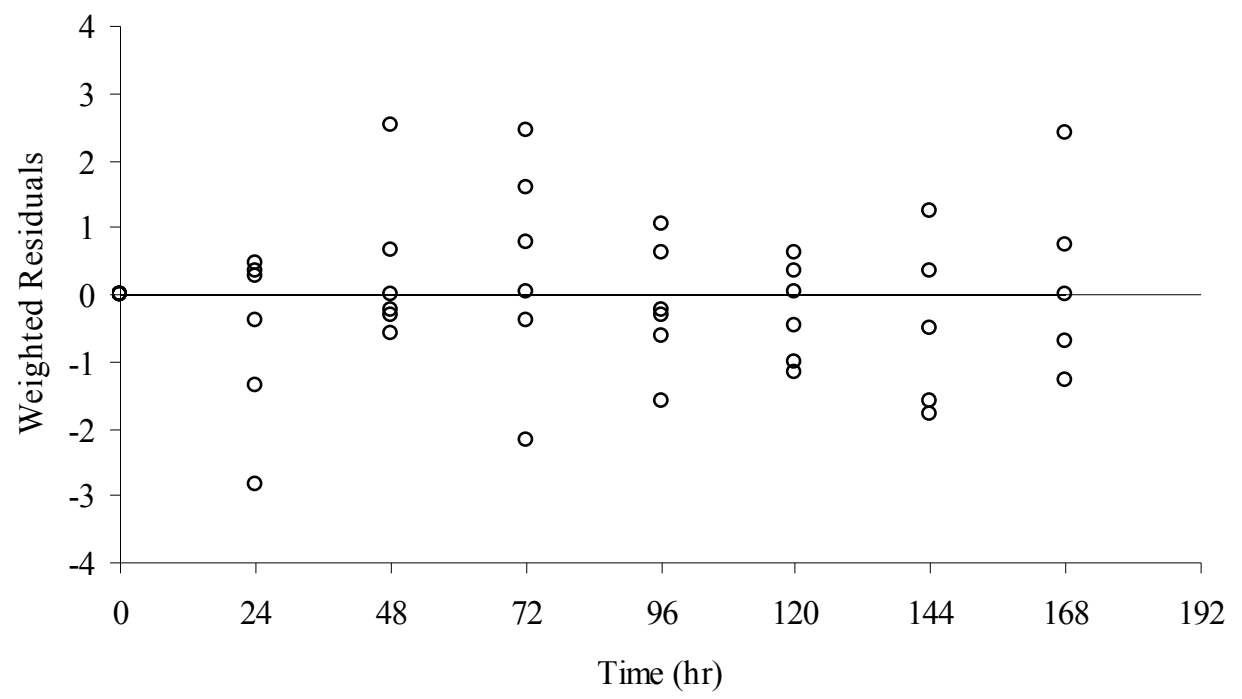

B)

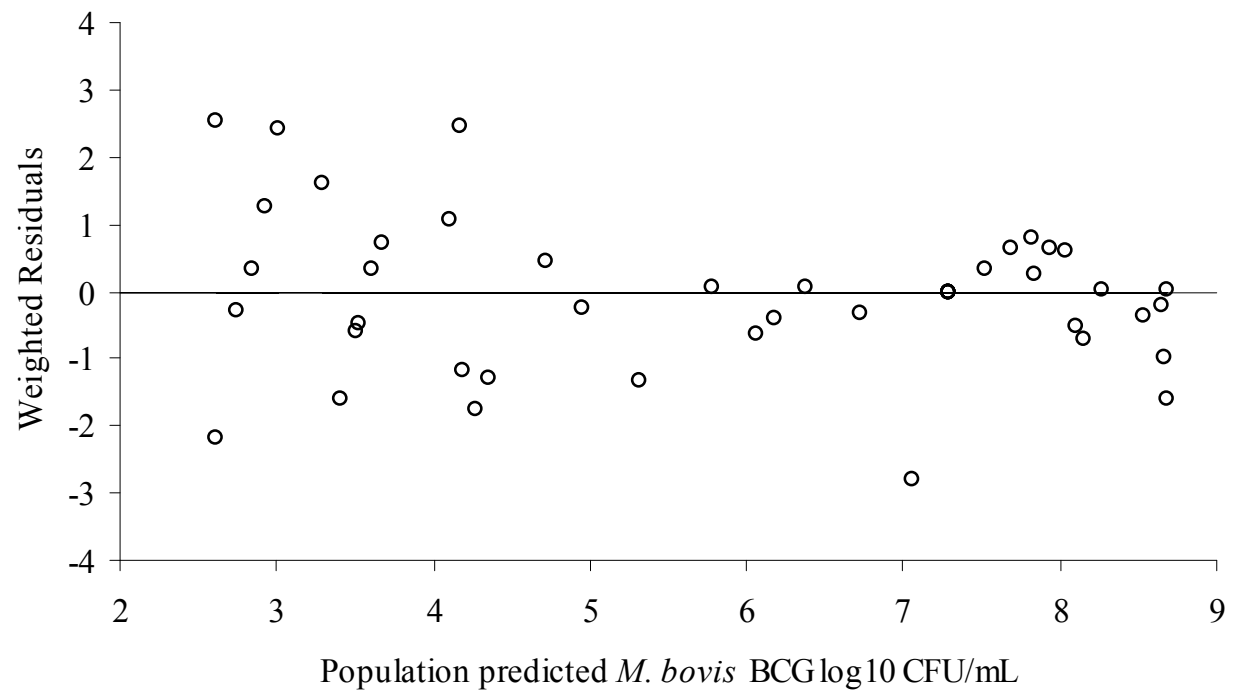

Figure 4-4. Goodness-of-Fit Plots for the Lee 1106 PK/PD Model - Weighted Residuals. Panel A shows weighted residuals vs. time (hr) and panel B shows weighted residuals vs. population predicted values $(\log 10 \mathrm{CFU} / \mathrm{mL})$. 
A)

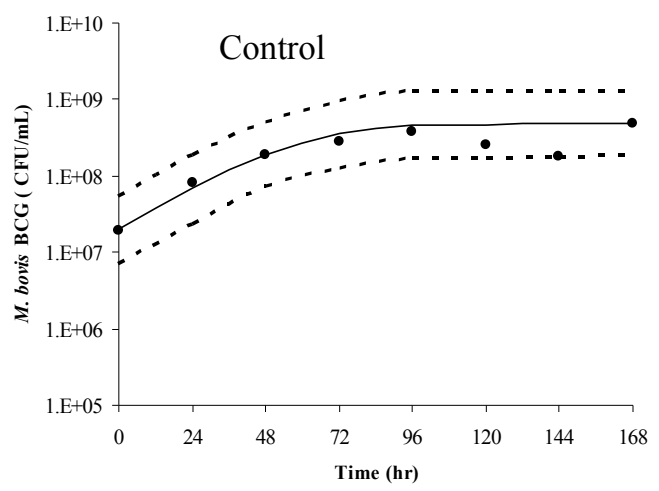

C)

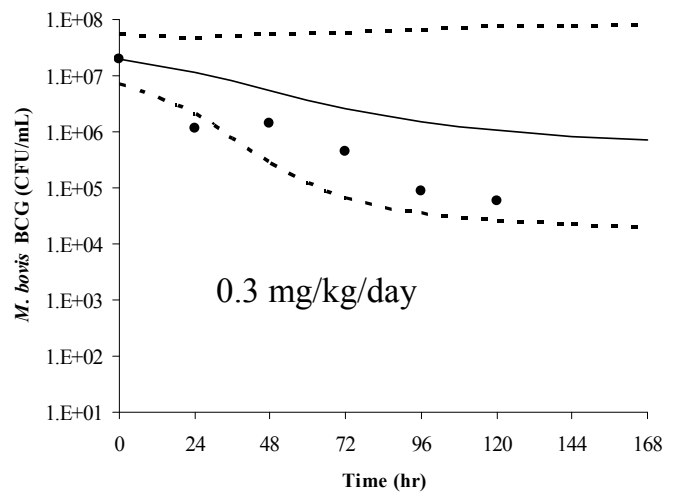

E)

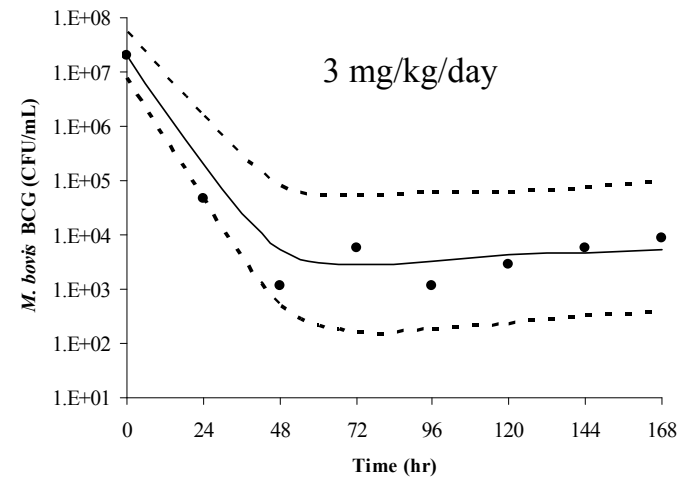

B)

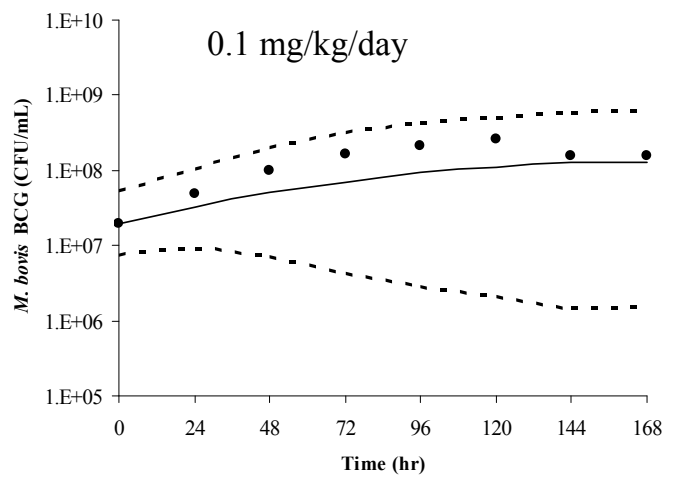

D)

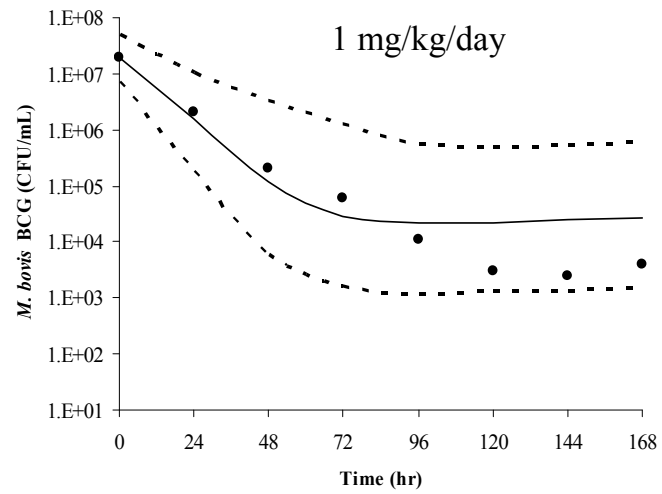

F)

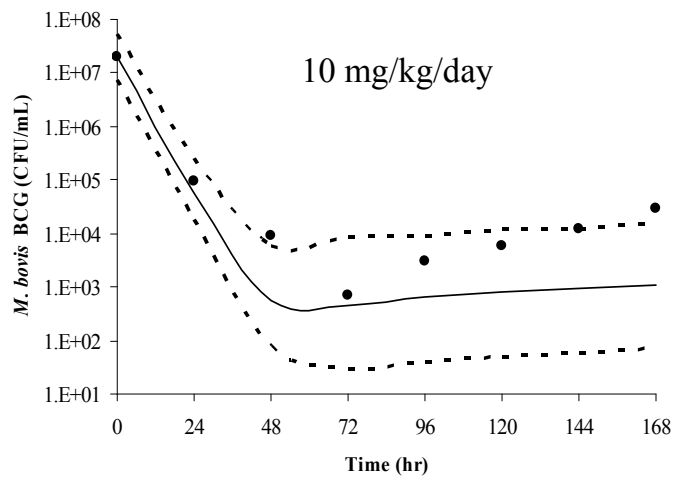

Figure 4-5. Visual Predictive Check for the PK/PD Model. 90\% Prediction intervals (dotted lines) for time-kill curves calculated from 1000 numerical simulations for each dosing regimen of Lee 1106. Continuous lines indicate median values and circles indicate the experimental M. bovis BCG cell counts (CFU/mL) at A) Control (no-drug), B) 0.1 $\mathrm{mg} / \mathrm{kg} /$ day regimen, C) $0.3 \mathrm{mg} / \mathrm{kg} /$ day regimen, D) $1 \mathrm{mg} / \mathrm{kg} /$ day regimen, E) $3 \mathrm{mg} / \mathrm{kg} /$ day regimen and F) $10 \mathrm{mg} / \mathrm{kg} /$ day regimen. 
A)

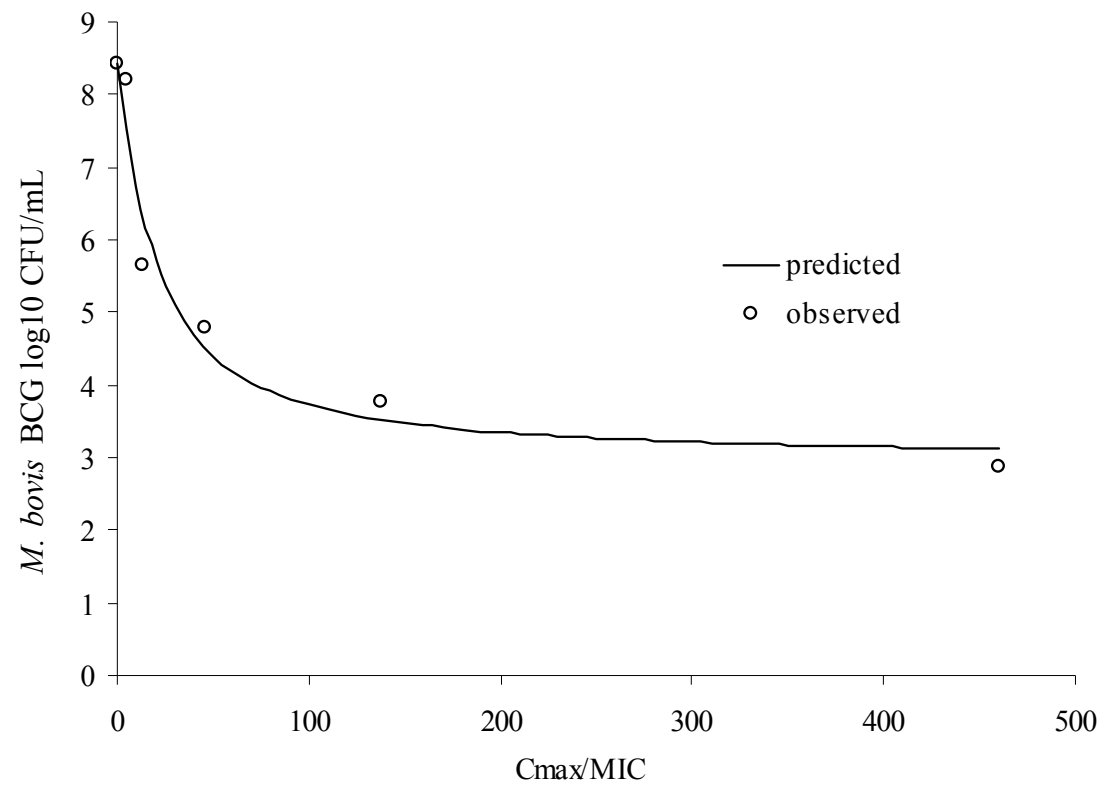

B)

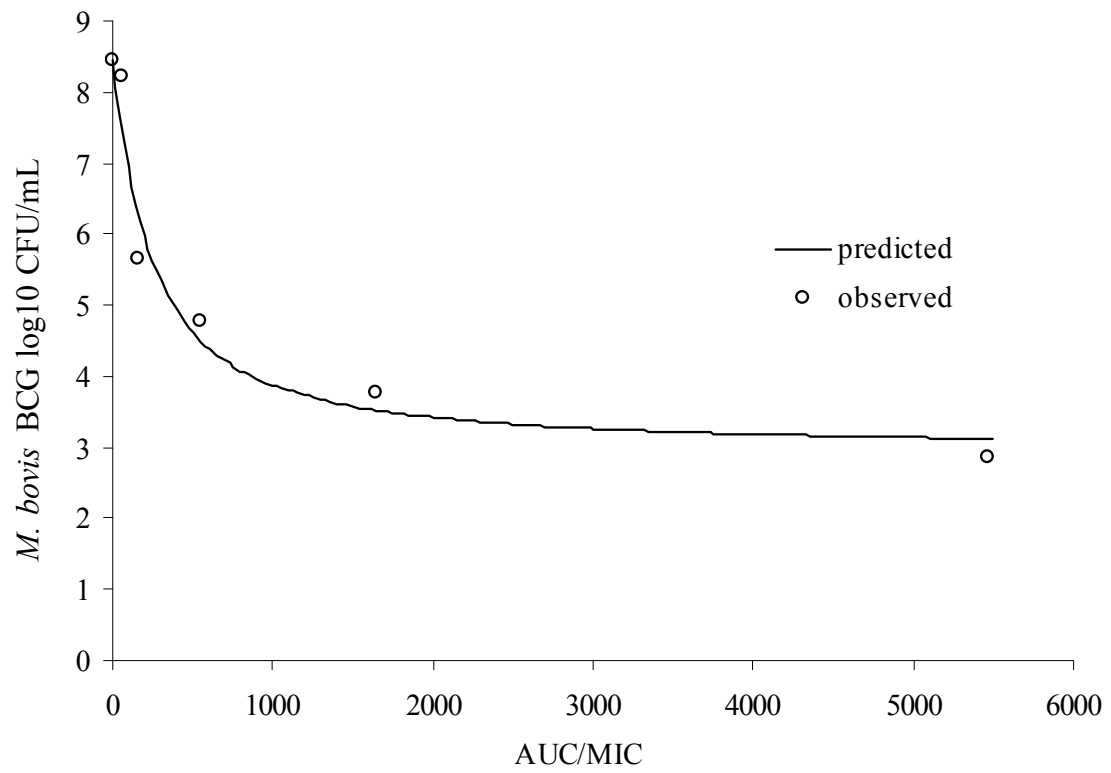

Figure 4-6. Relationship between PK/PD Indices and Microbial Kill on Day 3 after Treatment. Panels A and B show $\mathrm{C}_{\max } / \mathrm{MIC}$ and $\mathrm{AUC} / \mathrm{MIC}$, respectively. 
Table 4-3. Empirical PK/PD Indices Based on Day 3 Data.

\begin{tabular}{cccc}
\hline Parameter & Units & $\mathbf{A U C}_{\mathbf{0 - 2}} / \mathbf{M I C}$ & $\mathbf{C}_{\mathbf{m a x}} / \mathbf{M I C}$ \\
\hline $\mathbf{E}_{\mathbf{m a x}}$ & $\log 10 \mathrm{CFU} / \mathrm{mL}$ & 5.47 & 5.47 \\
$\mathbf{E C 5 0}$ & Variable dependent & 239 & 20.11 \\
$\mathbf{H}$ & on the index & 1.14 & 1.14 \\
$\mathbf{R}^{2}$ & & 0.995 & 0.995 \\
\hline
\end{tabular}

$\mathrm{R}^{2}$ is the coefficient of determination.

concentrations from all the dosing regimens (except $0.1 \mathrm{mg} / \mathrm{kg}$ ) were above the MIC for M. bovis $\mathrm{BCG}$ during one dosing interval.

For the simulation of time-kill curves for different dosing regimens in future in vivo efficacy studies in mice, the pharmacokinetics of Lee 1106 were extrapolated from rat to mouse using three-quarter exponent law in allometric scaling. The elimination halflife of Lee 1106 in mice was calculated as 5 hours. The numerical simulations using mouse PK data at the once daily (Figure 4-7) and twice daily dosing regimen (Figure 4-8) showed similar efficacy. The Lee 1106 regimens with once a week (Figure 4-9) and twice weekly (Figure 4-10) dosing predicted re-growth after an initial decrease in cell counts due to increased drug insensitive $M$. bovis BCG cell populations and may result in the ultimate selection of resistant populations of bacteria.

\section{Discussion}

Bacterial time-kill curve analysis captures the dynamic interplay between the bacteria and the drug by simulating the fluctuation in antibiotic concentrations observed in vivo. In contrast to a static parameter for antibacterial activity such as MIC, the timekill analysis provides an antibacterial agent's activity as a function of antibiotic concentration and time [201]. The in vitro PK/PD models offer several advantages in the determination of time-kill curves. First, time-kill curves can be determined at a wide range of concentrations which may not be possible with animal models or human studies. Secondly, dose-fractionation experimental designs can easily be implemented to remove the covariance between PK/PD indices to help determine the concentration- or timedependent killing nature of antibiotics. Thirdly, the lack of immune response in the in vitro $\mathrm{PK} / \mathrm{PD}$ model allows for a more accurate characterization of the interaction between the drug and the bacteria. Finally, it is relatively easy, inexpensive and ethical to determine time-kill profiles in in vitro models compared to in vivo models [206, 208].

The doubling time of $M$. bovis BCG of 12.2 hours is close to the published range, 13-20 hours, indicating that the in vitro model did not interfere with normal growth of $M$. bovis BCG [219]. Lee 1106 exhibited exposure dependent killing of $M$. bovis BCG which is evident from the initial kill rates analogous to early bactericidal activity (EBA) during 
A)

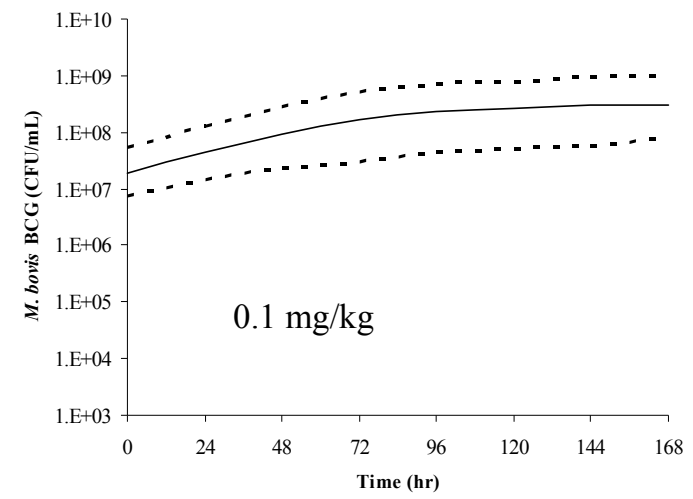

C)

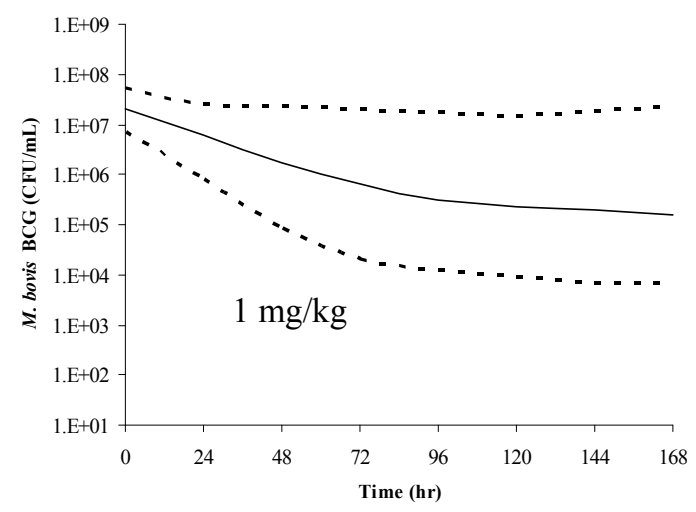

E)
B)

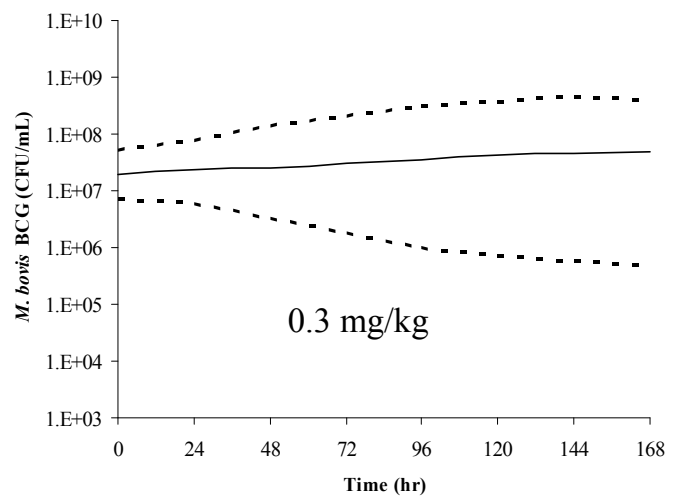

D)

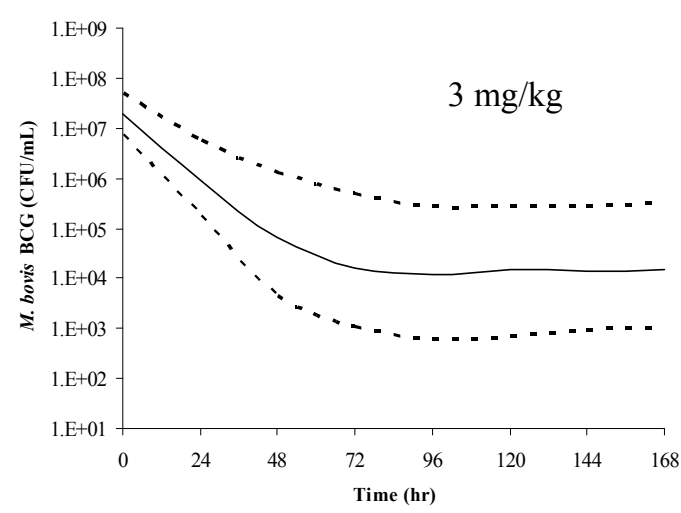

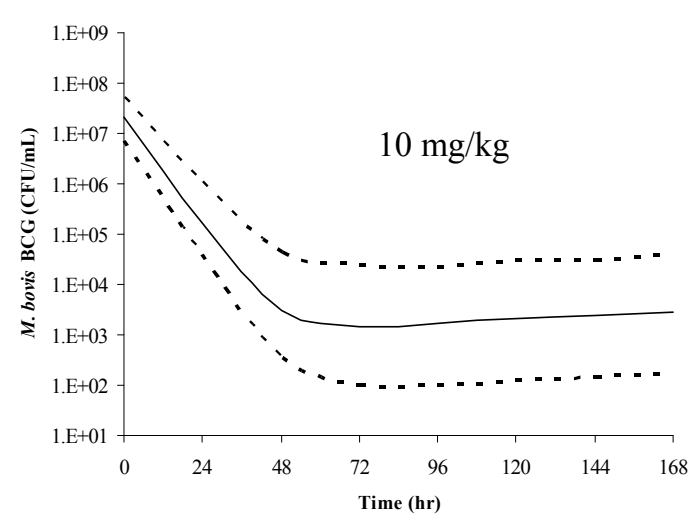

Figure 4-7. $\quad 90 \%$ Prediction Intervals (Dotted Lines) for Time-Kill Curves Calculated from 1000 Numerical Simulations for Once Daily Dosing Regimens of Lee 1106 in Mice. Continuous lines indicate median values. A) $0.1 \mathrm{mg} / \mathrm{kg}$ regimen, B) $0.3 \mathrm{mg} / \mathrm{kg}$ regimen, C) $1 \mathrm{mg} / \mathrm{kg}$ regimen, D) $3 \mathrm{mg} / \mathrm{kg}$ regimen and E) $10 \mathrm{mg} / \mathrm{kg}$ regimen. 
A)

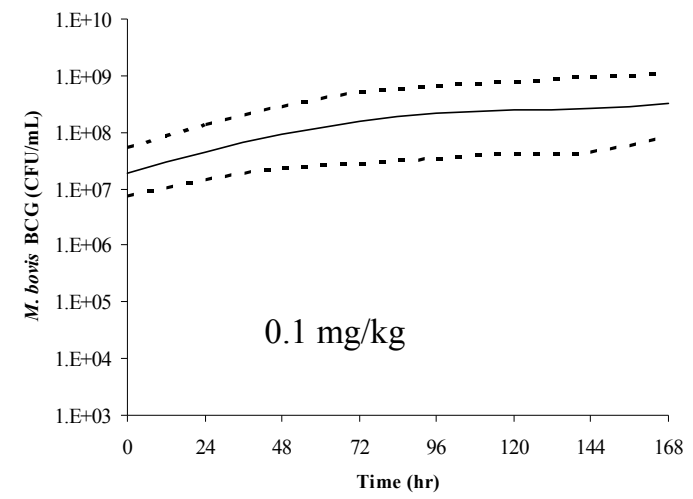

C)

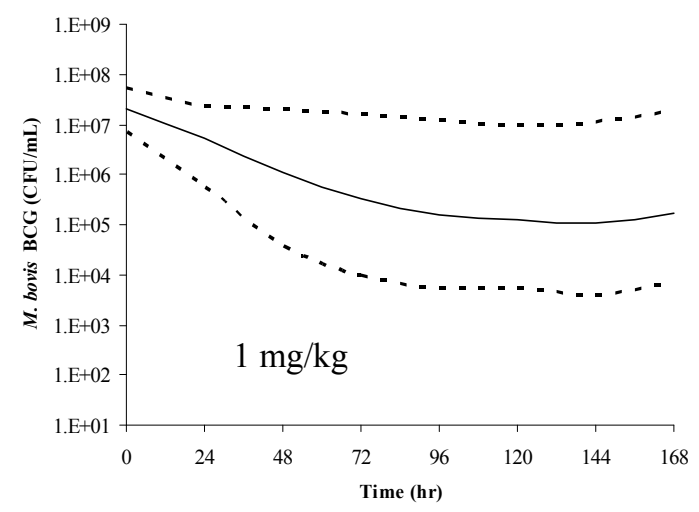

E)
B)

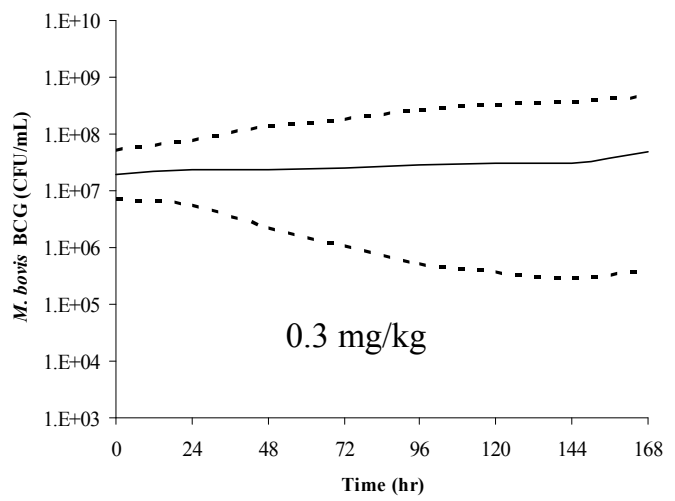

D)

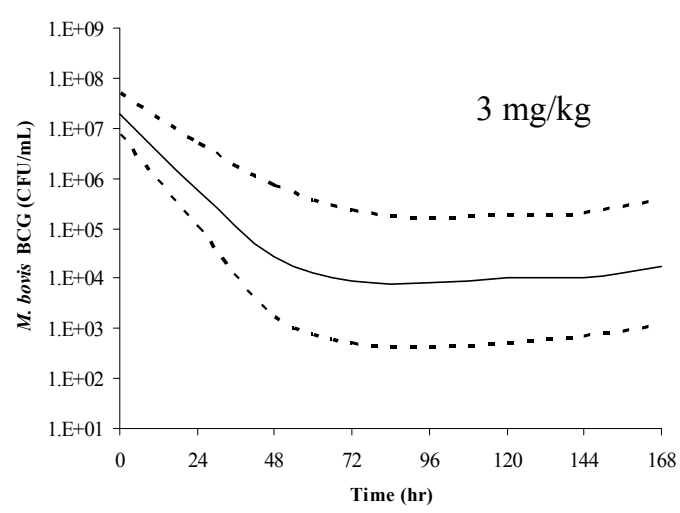

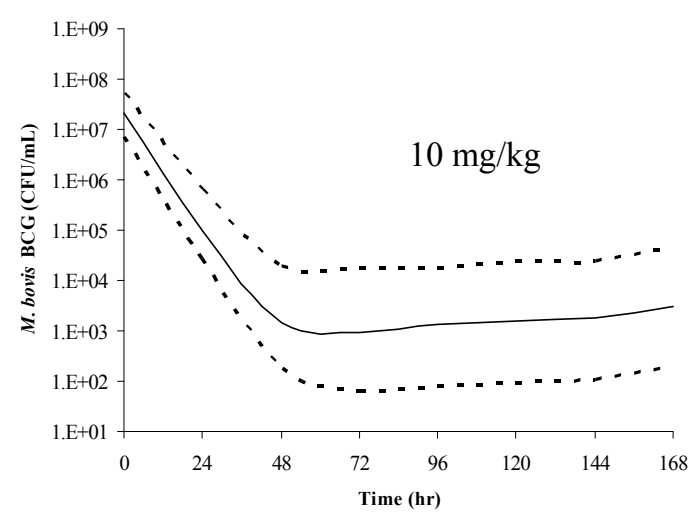

Figure 4-8. $\quad 90 \%$ Prediction Intervals (Dotted Lines) for Time-Kill Curves Calculated from 1000 Numerical Simulations for Twice Daily Dosing Regimens of Lee 1106 in Mice. Continuous lines indicate median values. A) $0.1 \mathrm{mg} / \mathrm{kg}$ regimen, B) $0.3 \mathrm{mg} / \mathrm{kg}$ regimen, C) $1 \mathrm{mg} / \mathrm{kg}$ regimen, D) $3 \mathrm{mg} / \mathrm{kg}$ regimen and E) $10 \mathrm{mg} / \mathrm{kg}$ regimen. 
A)

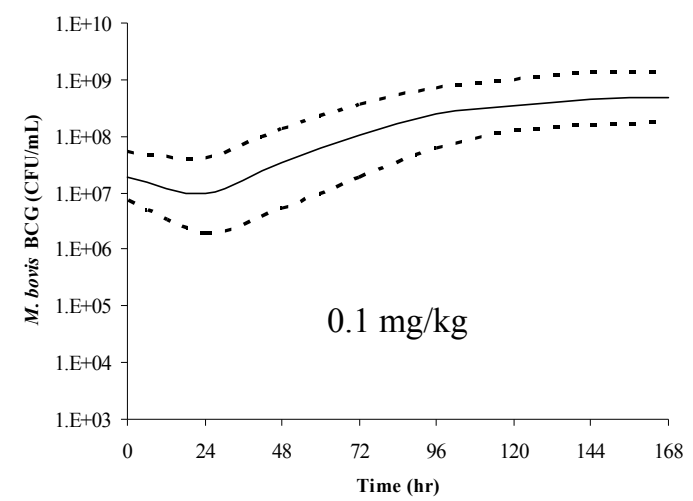

C)

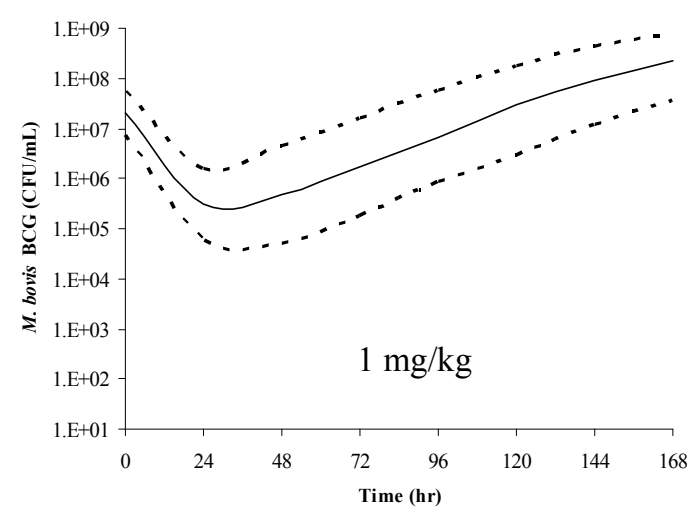

B)

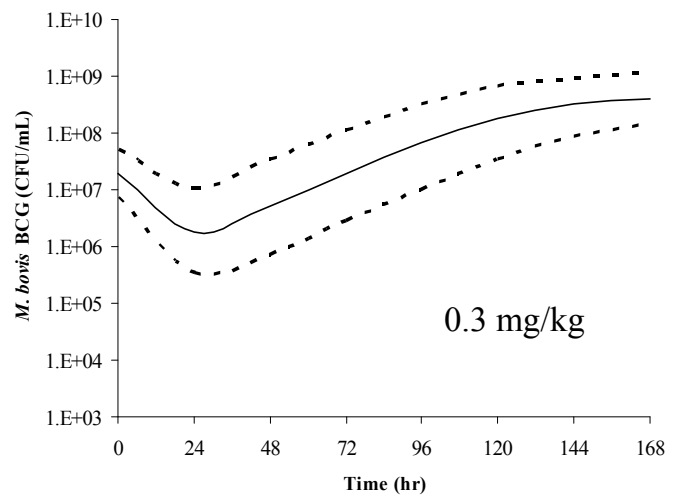

D)

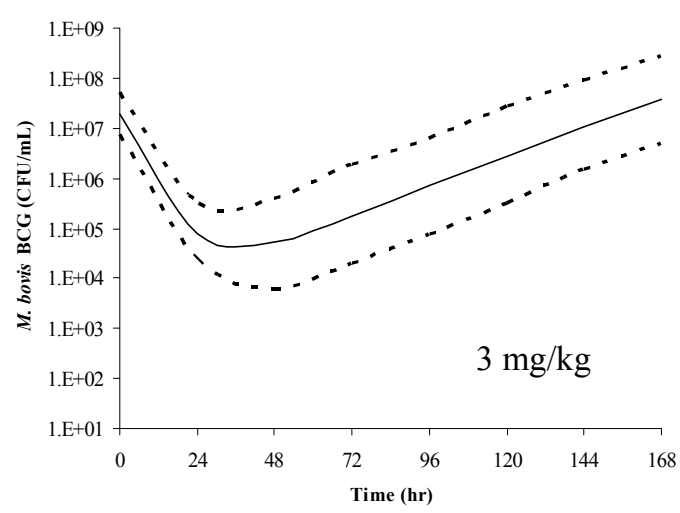

E)

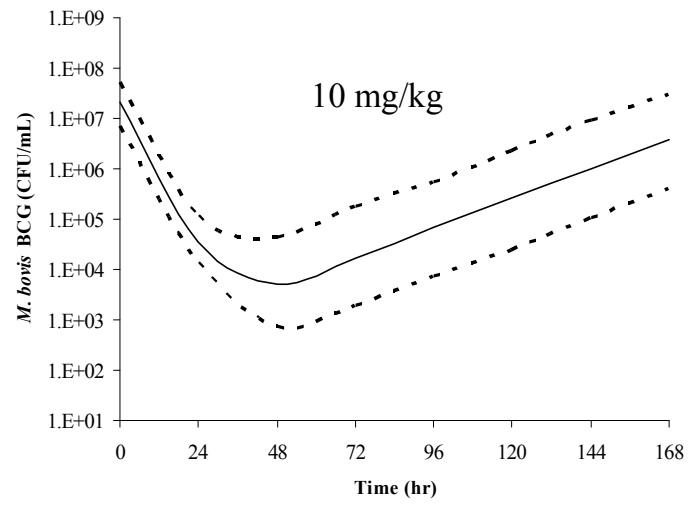

Figure 4-9. $\quad 90 \%$ Prediction Intervals (Dotted Lines) for Time-Kill Curves Calculated from 1000 Numerical Simulations for Once Weekly Dosing Regimens of Lee 1106 in Mice. Continuous lines indicate median values. A) $0.1 \mathrm{mg} / \mathrm{kg}$ regimen, B) $0.3 \mathrm{mg} / \mathrm{kg}$ regimen, C) $1 \mathrm{mg} / \mathrm{kg}$ regimen, D) $3 \mathrm{mg} / \mathrm{kg}$ regimen and E) $10 \mathrm{mg} / \mathrm{kg}$ regimen. 
A)

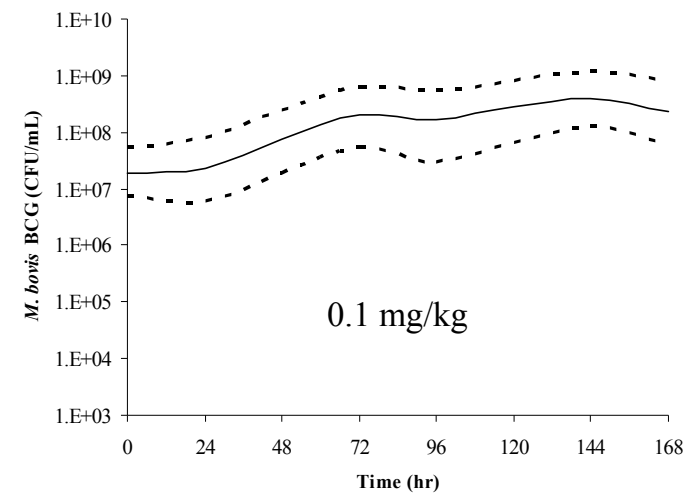

C)

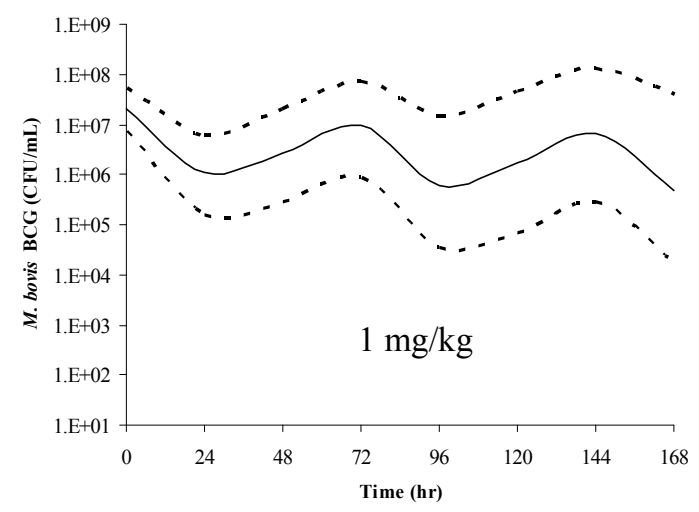

E)
B)

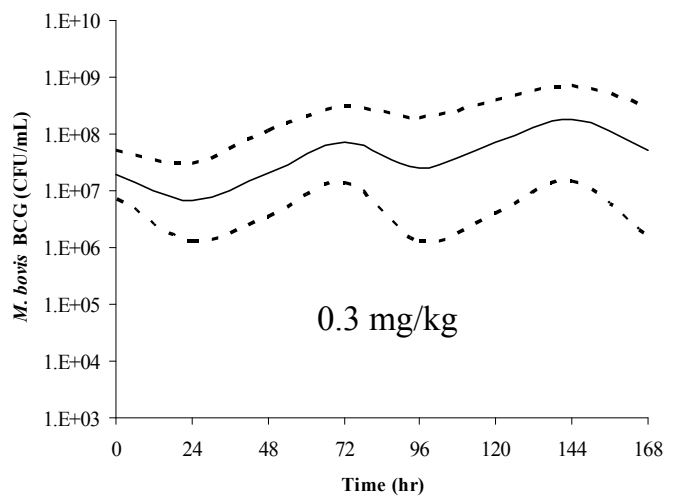

D)

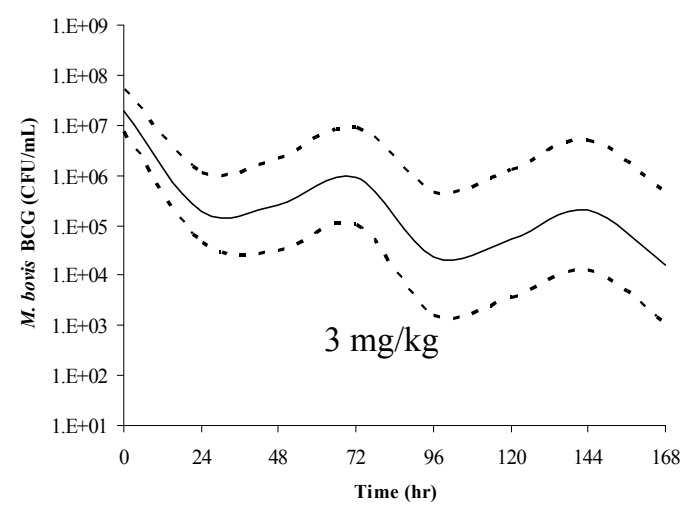

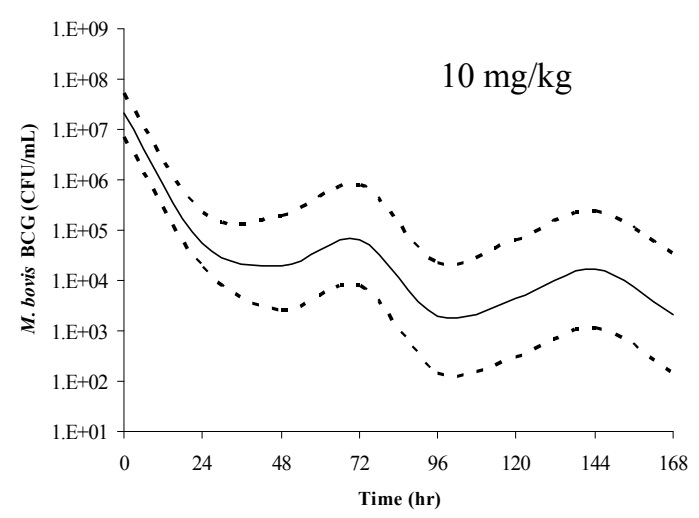

Figure 4-10. 90\% Prediction Intervals (Dotted Lines) for Time-Kill Curves Calculated from 1000 Numerical Simulations for Twice Weekly Dosing Regimens of Lee 1106 in Mice. Continuous lines indicate median values. A) $0.1 \mathrm{mg} / \mathrm{kg}$ regimen, B) $0.3 \mathrm{mg} / \mathrm{kg}$ regimen, C) $1 \mathrm{mg} / \mathrm{kg}$ regimen, D) $3 \mathrm{mg} / \mathrm{kg}$ regimen and E) $10 \mathrm{mg} / \mathrm{kg}$ regimen. 
the first two days of therapy. When compared to the most efficient anti-tuberculosis agent INH, Lee 1106 exhibited superior early bactericidal activity. Lee 1106 exhibited more than 2-log unit reduction $(2.12 \log 10 \mathrm{CFU} / \mathrm{mL} /$ day $)$ in viable count every day during the first two days of therapy. In comparison, INH (300 mg/day) exhibited only a $1.35 \mathrm{log}$ unit reduction in the viable count every day (Chapter 3). The Lee 1106-mediated killing effect reached a plateau at the $3 \mathrm{mg} / \mathrm{kg}$ /day dosing regimen. A further increase in dose to $10 \mathrm{mg} / \mathrm{kg}$ did not produce any increase in bactericidal activity. This could be due to a very broad dose range, $0.1 \mathrm{mg} / \mathrm{kg} /$ day to $10 \mathrm{mg} / \mathrm{kg} /$ day (100 fold) used in the study, which eventually reached the maximum possible bactericidal activity (Figure 4-2). Alternatively, this observation could indicate that Lee 1106 exhibits time-dependent killing of M. bovis BCG. However, no killing but bacterial growth instead was observed at the $0.1 \mathrm{mg} / \mathrm{kg}$ dosing regimen, even though the concentrations of Lee 1106 were above the MIC for M. bovis BCG $94.3 \%$ of time during one dosing interval.

The Lee 1106 mediated killing of bacteria ceased after 2-3 days of therapy at doses $3 \mathrm{mg} / \mathrm{kg}$ and $10 \mathrm{mg} / \mathrm{kg}$ once daily whereas the bactericidal activity lasted for 4-5 days at doses 0.3 and $1 \mathrm{mg} / \mathrm{kg}$ once daily. This difference in the bactericidal activity appears similar to the paradoxical 'Eagle effect' observed for $\beta$-lactam antibiotics which also exhibit reduced bactericidal effects at higher concentrations [237]. As a result, the re-growth of bacteria was observed after 3 days of therapy at the $3 \mathrm{mg} / \mathrm{kg}$ and $10 \mathrm{mg} / \mathrm{kg}$ dose levels and after 5 days of therapy at the $0.3 \mathrm{mg} / \mathrm{kg}$ and $1 \mathrm{mg} / \mathrm{kg}$ dose levels.

The PK/PD model with an adaptive IC50 function was able to describe the regrowth of bacteria after several days of drug exposure at all the studied dose groups. The increase in IC50 is inversely related the Lee 1106 exposure and the ratio of cell counts to the initial cell numbers (Eq. 4-2). This means that low exposure to Lee 1106 results in increased development of insensitivity, manifested as an increase in apparent IC50. The IC50 was estimated as $0.55 \mathrm{mg} / \mathrm{L}$ and increased over the course of treatment to 31.42 $\mathrm{mg} / \mathrm{L}$. Total concentrations of Lee 1106 were utilized in developing the PK/PD model assuming that the extent of binding to proteins (BSA, bovine serum albumin) in the medium is similar to that of plasma protein binding. Middlebrook $7 \mathrm{H} 9$ medium contains $5 \mathrm{~g} / \mathrm{L}$ of BSA whereas the albumin in the plasma ranges from 30 to $50 \mathrm{~g} / \mathrm{L}$. The stationary concentration of Lee 1106 which is defined as the concentration at which the rate of bacterial killing equals growth was calculated using IC50 as described by Mouton et al. [233]. Usually, the stationary concentration is lower than the MIC for antibiotics with concentration-dependent kill. On the contrary, the calculated stationary concentration for Lee $1106,0.111 \mathrm{mg} / \mathrm{L}$, was greater than the MIC, $0.025 \mathrm{mg} / \mathrm{L}$. This observation is in agreement with the anti-mycobacterial activity of other compounds in the nitrofuranylamide series. These compounds showed bacteriostatic effect at concentrations equivalent to $\mathrm{MIC}$ and bactericidal effect at much higher concentrations ranging from 0.1 to $1.56 \mathrm{mg} / \mathrm{L}$ [234]. The percentage of time the concentration of Lee 1106 is above the stationary concentration for $M$. bovis BCG at $0.1 \mathrm{mg} / \mathrm{kg}$ dosing regimen in a dosing interval is only $1.67 \%$, a likely reason for the absence of bactericidal effect at this dosing regimen. 
The empirical PK/PD analysis revealed that Lee 1106 exhibits an exposuredependent antibacterial kill. The PK/PD indices AUC/MIC and $\mathrm{C}_{\max } / \mathrm{MIC}$ both could explain the observed Lee 1106-mediated bacterial kill $\left(\mathrm{R}^{2}=0.995\right)$.

Since the aerosol infection mouse model of TB is used widely for the evaluation of antimycobacterial activity, pharmacokinetics of Lee 1106 were extrapolated from rat to mouse by using allometric scaling. The scaling from rat to mouse was performed under an assumption that the metabolic activity and thus the clearance of the compound follows the three-quarter exponent law [238, 239]. Accordingly, an elimination half-life of 5 hours in mice as opposed to 10.3 hours in rats was used in the numerical simulations for predicting bactericidal effect of Lee 1106 in the mouse model. The results of numerical simulation experiments indicate that the twice daily dosing regimens produce a bactericidal effect similar to the once daily dosing regimens. Furthermore, twice weekly and once weekly dosing regimens predicted increased drug insensitive populations of bacteria manifested as re-growth in the predicted time-kill curves. These simulations suggest that the once daily dosing regimen is adequate for the further in vivo studies in mouse models of tuberculosis.

\section{Conclusion}

In conclusion, we determined the time-kill profiles for dosing regimens of Lee 1106 against a slow growing organism, M. bovis BCG. The PK/PD model with an adaptive IC50 function was able to describe the behavior in the data along with the regrowth observed after several days of exposure in the time-kill profiles. The model-based numerical simulations indicate that the once daily dosing regimen in the mouse could produce similar antibacterial effects as observed in the in vitro kill curve analysis. 


\section{CHAPTER 5. SUMMARY}

Currently used strategies for the treatment of tuberculosis involve administration of multiple drugs for a minimum of 6 to 9 months. However, these prolonged regimens do not always achieve sterilization, as evidenced by post-therapy relapse in a subgroup of treated individuals [2]. Treatment of multi drug resistant-TB (MDR-TB) is 100 times costlier than drug sensitive TB, increases the duration of therapy and necessitates the use of more toxic second-line drugs [1]. Recently, the emergence of XDR-TB (extensively drug resistant TB) cases worldwide is increasing concerns that we may lose control over the spread of TB $[1,3]$. Though currently there are intensive efforts to develop new TB therapies, it is worth noting that no new major classes of drugs have been approved for the treatment of TB since the introduction of the rifamycins into the market in 1971 [188]. This indifference to the burgeoning drug resistance problem stemmed from the perceived lack of commercial incentive in the development of new TB drugs. As a result, a majority of the ongoing efforts in the development of novel therapeutic agents were initiated by non-profit organizations and academic research institutions [14].

In an effort to develop novel therapeutic agents for TB a new class of chemical agents, nitrofuranylamides, is being developed by our group [189-192, 195]. In the current dissertation, I hypothesized that application of an iterative PK/PD-guided approach would facilitate the identification of antimycobacterial lead compounds suitable for further development. I investigated this hypothesis for the class of chemical agents being developed by our group. First, I developed an iterative pharmacokinetically-guided lead optimization paradigm and successfully applied this approach in the optimization of nitrofuranylamide lead compounds. To complement the PK guided approach and expand it to a PK/PD-guided lead optimization paradigm, I developed a novel in vitro PK/PD model for characterizing the time-kill profiles of lead compounds against mycobacteria. I validated the in vitro model by characterizing the effect of the first-line anti-tuberculosis agent isoniazid against $M$. bovis BCG as a model organism. Lastly, I determined time-kill profiles for dosing regimens of a nitrofuranylamide lead compound, Lee 1106 in the in vitro model. I then developed a mathematical model for describing the Lee 1106 mediated bacterial kill. Based on this mathematical model, I performed numerical simulations for predicting the bactericidal effect of Lee 1106 at different, untested dosing regimens and gained further insights into the in vivo antimycobacterial activity of Lee 1106.

In the first step, I examined the biopharmaceutic properties and preclinical pharmacokinetics of nitrofuranylamides to accelerate the optimization of leads into development candidates. All tested compounds had high in vitro antimicrobial activity. The first tested compound, Lee 562, exhibited a high systemic clearance, short terminal half-life, and low oral bioavailability of $15.9 \%$. These observations were further supported by the poor metabolic stability of Lee 562, which was attributed to its amide linkage and the benzyl piperazine side chain in the structure (Figure 2-1) Thus, two second generation follow-up compounds, Lee 878 and Lee 952 were tested that included structural modifications for increased metabolic stability. For Lee 878, the amide bond 
was replaced by an isoxazole ring; for Lee 952, the amide hydrogen was substituted with a methyl group and the benzyl piperazine was replaced with a solubilizing morpholino group. Both compounds showed improved metabolic stability in a rat liver microsomal stability assay compared to Lee 562, with Lee 878 being much more stable than Lee 952. As expected, this in vitro observation translated into an increased in vivo stability (lower plasma clearance) of Lee 878 compared to Lee 562 and Lee 952, with a 20- and 10-fold higher systemic exposure, respectively. As a consequence, oral bioavailability of Lee 878 reached $\sim 27 \%$ compared to $16 \%$ for Lee 952 and Lee 562 . Since the microsomal metabolic stability was found to be correlated with the oral bioavailability of the first and second generation compounds we tested, a new set of nine third generation compounds with high in vitro activity belonging to the same series was screened for microsomal metabolic stability to guide selection of a stable compound for pharmacokinetic evaluation. The most stable compound in the assay, Lee 1106 was selected for further PK evaluation in rats. The compound exhibited favorable pharmacokinetic properties such as a low systemic clearance and a long terminal half-life. However, oral bioavailability of Lee 1106 was limited (4.6\%). Biopharmaceutic evaluation of the compound showed that the compound has poor aqueous solubility and a high clogP. The compound also exhibited high plasma protein binding. All three qualities, poor aqueous solubility, high clogP and high plasma protein binding suggest hydrophobicity of the molecule. Based on these results, a screening paradigm (Figure 2-8) was developed for optimization of the nitrofuranylamide lead compounds in a timely and cost-effective manner [235]. The lead compound Lee 1106 was selected for further evaluation in an in vitro PK/PD model.

In order to develop a PK/PD guided approach to lead optimization, a simple, inexpensive and reliable in vitro assay for the pharmacodynamic assessment of novel antibiotics is very much needed. Considering the slow growing nature of $M$. tuberculosis and the lack of availability of suitable pharmacodynamic models for assessing the activity of antimicrobials against slow growing organisms, I developed a novel in vitro $\mathrm{PK} / \mathrm{PD}$ model and evaluated its performance by studying the pharmacodynamic effect of a first line antituberculosis drug, isoniazid (INH) against $M$. bovis BCG as a model organism. In the in vitro model $M$. bovis BCG was exposed to drug concentration-time profiles as usually encountered in humans during multiple dose therapy. Simulated INH dosing regimens included 25, 100 and $300 \mathrm{mg}$ /day. Concentration-time profiles were simulated for both human INH metabolism phenotypes, fast as well as slow metabolizers secondary to NAT2 polymorphism. The generated time-kill data was analyzed using a semimechanistic pharmacokinetic-pharmacodynamic model that included an adaptive IC50 function for explaining the re-growth of bacteria observed over the course of treatment. The PK/PD model was able to describe the data well. Empirical PK/PD analysis revealed that the $\mathrm{AUC}_{0-24} / \mathrm{MIC}$ is the most explanatory $\mathrm{PK} / \mathrm{PD}$ index for the antimicrobial effect of $\mathrm{INH}$ on $M$. bovis BCG. The exposure dependent killing of bacteria by $\mathrm{INH}$ is in agreement with similar in vitro and in vivo studies using $M$. tuberculosis as test organism $[167,212,215]$. These findings suggest that the proposed in vitro PK/PD model and associated modeling approach are valuable tools for the assessment of antibacterial activity in slow growing microorganisms. 
After successful application for INH, the in vitro model was used to investigate the time-kill behavior of the novel lead compound, Lee 1106. Lee 1106 showed exposure-dependent killing of M. bovis BCG. Empirical PK/PD analysis identified the $\mathrm{PK} / \mathrm{PD}$ indices $\mathrm{AUC} / \mathrm{MIC}$ and $\mathrm{C}_{\max } / \mathrm{MIC}$ as predictors of the bactericidal effect of Lee 1106. Numerical simulation experiments were performed using the PK/PD model to predict the kill effect of different untested multiple dose regimens of Lee 1106 in mice. The pharmacokinetics of Lee 1106 were extrapolated from rats to mice by allometric scaling. The mouse PK was used in these simulations since the mouse models of TB are widely used for assessing the efficacy of anti-TB compounds. The simulation experiments suggest that once daily dosing would be adequate for in vivo efficacy studies in mouse models. The time-kill curves obtained by numerical simulation will guide the selection of dosing regimens for future in vivo experiments (M.tb mouse infection models).

In summary, we have successfully developed an iterative PK/PD guided process for lead optimization of nitrofuranylamides that uses a set of biopharmaceutic, pharmacokinetic and pharmacodynamic evaluations designed to identify the compounds suitable for further development. The utilization of PK/PD principles is believed to result in a scientifically driven, evidence-based, more focused and accelerated drug development process. Knowledge about the mechanistic reasons for lack of in vivo efficacy are of particular importance in guiding the chemical lead optimization process and to pinpoint the potential molecular modifications a compound has to undergo in order to have bioactivity and drug-likeness, i.e., the set of molecular properties and structural features that determine whether a compound is likely to succeed in drug development. The results of this study serve as an example for the optimization of lead compounds against tuberculosis. The general approach described in this dissertation, however, can also be applied in the optimization of leads in other chemical classes for treating tuberculosis and as well as other bacterial infections of slow as well as fast growing microorganisms. 


\section{LIST OF REFERENCES}

1. World Health Organization, Fact sheet No 104: Tuberculosis. 2007, Geneva: WHO.

2. El-Sadr, W.M., et al., A review of efficacy studies of 6-month short-course therapy for tuberculosis among patients infected with human immunodeficiency virus: differences in study outcomes. Clin Infect Dis, 2001. 32(4): p. 623-32.

3. U.S. Department of Health and Human Services Center for Disease Control and Prevention, Emergence of Mycobacterium tuberculosis with extensive resistance to second-line drugs--worldwide, 2000-2004. MMWR Morb Mortal Wkly Rep, 2006. 55(11): p. 301-5.

4. Wenzel, R.P., The antibiotic pipeline--challenges, costs, and values. N Engl J Med, 2004. 351(6): p. 523-26.

5. Spigelman, M. and S. Gillespie, Tuberculosis drug development pipeline: progress and hope. Lancet, 2006. 367(9514): p. 945-47.

6. Kerns, E.H., High throughput physicochemical profiling for drug discovery. J Pharm Sci, 2001. 90(11): p. 1838-58.

7. Prentis, R.A., Y. Lis, and S.R. Walker, Pharmaceutical innovation by the seven UK-owned pharmaceutical companies (1964-1985). Br J Clin Pharmacol, 1988. 25(3): p. 387-96.

8. van De Waterbeemd, H., et al., Property-based design: optimization of drug absorption and pharmacokinetics. J Med Chem, 2001. 44(9): p. 1313-33.

9. Veber, D.F., et al., Molecular properties that influence the oral bioavailability of drug candidates. J Med Chem, 2002. 45(12): p. 2615-23.

10. Venkatesh, S. and R.A. Lipper, Role of the development scientist in compound lead selection and optimization. J Pharm Sci, 2000. 89(2): p. 145-54.

11. Pritchard, J.F., et al., Making better drugs: Decision gates in non-clinical drug development. Nat Rev Drug Discov, 2003. 2(7): p. 542-53.

12. Caldwell, G.W., et al., The new pre-preclinical paradigm: compound optimization in early and late phase drug discovery. Curr Top Med Chem, 2001. 1(5): p. 353-66.

13. Volker, C. and J.R. Brown, Bioinformatics and the discovery of novel antimicrobial targets. Curr Drug Targets Infect Disord, 2002. 2(4): p. 279-90.

14. Global Alliance for TB Drug Development. 2007 [cited May 23, 2007]; Available from: http://www.tballiance.org.

15. Global Alliance for TB Drug Development, Tuberculosis. Scientific blueprint for tuberculosis drug development. Tuberculosis (Edinb), 2001. 81 Suppl 1: p. 1-52.

16. Solotorovsky, M., et al., Pyrazinoic acid amide: An agent active against experimental murine tuberculosis. Proc Soc Exp Biol Med, 1952. 79(4): p. 56365.

17. Cole, S.T., et al., Deciphering the biology of Mycobacterium tuberculosis from the complete genome sequence. Nature, 1998. 393(6685): p. 537-44.

18. Banerjee, A., et al., inhA, a gene encoding a target for isoniazid and ethionamide in Mycobacterium tuberculosis. Science, 1994. 263(5144): p. 227-30. 
19. Oliveira, J.S., et al., Crystallographic and pre-steady-state kinetics studies on binding of $N A D H$ to wild-type and isoniazid-resistant enoyl-ACP $(C o A)$ reductase enzymes from Mycobacterium tuberculosis. J Mol Biol, 2006. 359(3): p. 646-66.

20. Campbell, E.A., et al., Structural mechanism for rifampicin inhibition of bacterial rna polymerase. Cell, 2001. 104(6): p. 901-12.

21. Zhang, Y. and D. Mitchison, The curious characteristics of pyrazinamide: a review. Int J Tuberc Lung Dis, 2003. 7(1): p. 6-21.

22. Belanger, A.E., et al., The embAB genes of Mycobacterium avium encode an arabinosyl transferase involved in cell wall arabinan biosynthesis that is the target for the antimycobacterial drug ethambutol. Proc Natl Acad Sci U S A, 1996. 93(21): p. 11919-24.

23. Spotts, C.R. and R.Y. Stanier, Mechanism of streptomycin action on bacteria: a unitary hypothesis. Nature, 1961. 192: p. 633-37.

24. Zhang, Y., The magic bullets and tuberculosis drug targets. Annu Rev Pharmacol Toxicol, 2005. 45: p. 529-64.

25. Kondo, J., et al., Crystal structure of the bacterial ribosomal decoding site complexed with amikacin containing the gamma-amino-alpha-hydroxybutyryl (haba) group. Biochimie, 2006. 88(8): p. 1027-31.

26. Aubry, A., et al., Mycobacterium tuberculosis DNA gyrase: interaction with quinolones and correlation with antimycobacterial drug activity. Antimicrob Agents Chemother, 2004. 48(4): p. 1281-88.

27. Rengarajan, J., et al., The folate pathway is a target for resistance to the drug para-aminosalicylic acid (PAS) in mycobacteria. Mol Microbiol, 2004. 53(1): p. 275-82.

28. Sampathkumar, P., et al., Structure of the Mycobacterium tuberculosis flavin dependent thymidylate synthase (MtbThyX) at 2.0A resolution. J Mol Biol, 2005. 352(5): p. 1091-104.

29. LeMagueres, P., et al., The 1.9 A crystal structure of alanine racemase from Mycobacterium tuberculosis contains a conserved entryway into the active site. Biochemistry, 2005. 44(5): p. 1471-81.

30. Aranda, C.P., Second-line agents: p-amino salicylic acid, ethionamide, cycloserine and thiacetazone, in Tuberculosis, Rom, WN and Garay, SM; Editors. 1997, Lippincott-Raven: Philadelphia, PA. p. 811-16.

31. Petri, W.A., Chemotherapy of tuberculosis, Mycobacetrium avium complex disease, and leprosy, in Goodman and Gilman's The Pharmacological Basis of Therapeutics, Brunton, LL; Lazo, JS; and Parke, KL; Editors. 2006, McGrawHill: New York, NY. p. 1203-24.

32. Mdluli, K. and M. Spigelman, Novel ta.rgets for tuberculosis drug discovery. Curr Opin Pharmacol, 2006. 6(5): p. 459-67.

33. Zhang, Y., K. Post-Martens, and S. Denkin, New drug candidates and therapeutic targets for tuberculosis therapy. Drug Discov Today, 2006. 11(1-2): p. 21-27.

34. Zhang, Y. and L.M. Amzel, Tuberculosis drug targets. Curr Drug Targets, 2002. 3(2): p. 131-54.

35. Bloch, K. and D. Vance, Control mechanisms in the synthesis of saturated fatty acids. Annu Rev Biochem, 1977. 46: p. 263-98. 
36. Sullivan, T.J., et al., High affinity InhA inhibitors with activity against drugresistant strains of Mycobacterium tuberculosis. ACS Chem Biol, 2006. 1(1): p. 43-53.

37. Alderwick, L.J., et al., Identification of a novel arabinofuranosyltransferase (AftA) involved in cell wall arabinan biosynthesis in Mycobacterium tuberculosis. J Biol Chem, 2006. 281(23): p. 15653-61.

38. Pan, F., et al., Cell wall core galactofuran synthesis is essential for growth of mycobacteria. J Bacteriol, 2001. 183(13): p. 3991-98.

39. Kremer, L., et al., Galactan biosynthesis in Mycobacterium tuberculosis. Identification of a bifunctional UDP-galactofuranosyltransferase. J Biol Chem, 2001. 276(28): p. 26430-40.

40. Li, W., et al., rmlB and rmlC genes are essential for growth of mycobacteria. Biochem Biophys Res Commun, 2006. 342(1): p. 170-78.

41. Ginsburg, A.S., J.H. Grosset, and W.R. Bishai, Fluoroquinolones, tuberculosis, and resistance. Lancet Infect Dis, 2003. 3(7): p. 432-42.

42. Cynamon, M.H., et al., Activities of several novel oxazolidinones against Mycobacterium tuberculosis in a murine model. Antimicrob Agents Chemother, 1999. 43(5): p. 1189-91.

43. Andries, K., et al., A diarylquinoline drug active on the ATP synthase of Mycobacterium tuberculosis. Science, 2005. 307(5707): p. 223-27.

44. Munoz-Elias, E.J. and J.D. McKinney, Mycobacterium tuberculosis isocitrate lyases 1 and 2 are jointly required for in vivo growth and virulence. Nat Med, 2005. 11(6): p. 638-44.

45. Nunn, P., et al., Cutaneous hypersensitivity reactions due to thiacetazone in HIV1 seropositive patients treated for tuberculosis. Lancet, 1991. 337(8742): p. 62730.

46. Amidon, G.L., et al., A theoretical basis for a biopharmaceutic drug classification: the correlation of in vitro drug product dissolution and in vivo bioavailability. Pharm Res, 1995. 12(3): p. 413-20.

47. Lipinski, C.A., Drug-like properties and the causes of poor solubility and poor permeability. J Pharmacol Toxicol Methods, 2000. 44(1): p. 235-49.

48. van De Waterbeemd, H., The fundamental variables of the biopharmaceutics classification system (BCS): a commentary. Eur J Pharm Sci, 1998. 7(1): p. 1-3.

49. Avdeef, A. and B. Testa, Physicochemical profiling in drug research: a brief survey of the state-of-the-art of experimental techniques. Cell Mol Life Sci, 2002. 59(10): p. 1681-89.

50. Avdeef, A., Physicochemical profiling (solubility, permeability and charge state). Curr Top Med Chem, 2001. 1(4): p. 277-351.

51. Kasim, N.A., et al., Molecular properties of WHO essential drugs and provisional biopharmaceutical classification. Mol Pharm, 2004. 1(1): p. 85-96.

52. Meibohm, B. and H. Derendorf, Basic concepts of pharmacokinetic/pharmacodynamic (PK/PD) modelling. Int J Clin Pharmacol Ther, 1997. 35(10): p. 401-13.

53. Del Rio-Estrada, C. and H.W. Dougherty, Kirk-Othmer Encyclopedia of Chemical Technology. 2nd ed. Vol. 21. 1970: John Wliey \& Sons: Hoboken, NJ. p. 528-33. 
54. Wishart, D.S., et al., DrugBank: a comprehensive resource for in silico drug discovery and exploration. Nucleic Acids Res, 2006. 34(Database issue): p. D668-72.

55. Furesz, S., Chemical and biological properties of rifampicin. Antibiot Chemother, 1970. 16: p. 316-51.

56. Girgis, E.H., Z. Gad, and M. Maram, Ion-pair extraction and precipitation methods for ethambutol determination. J Pharm Sci, 1974. 63(11): p. 1764-67.

57. Mossa, J.S., A.H.U.K. Taragan, and M.M.A. Hasan, Streptomycin, in Analytical Profiles of Drug Substances. 1987, Academic Press: London, UK. p. 507-52.

58. Mossa, J.S., A.H.U.K. Taragan, and M.M.A. Hasan, Kanamycin, in Analytical Profiles of Drug Substances. 1987, Academic Press: London, UK. p. 260-88.

59. Hasan, M.M.A., A.I. Jado, and M.U. Zubair, Aminosalicylic acid, in Analytical Profiles of Drug Substances. 1981, Academic Press: London, UK. p. 1-15.

60. Lamb, J.W., Cycloserine, in Analytical Profiles of Drug Substances. 1987, Academic Press: London, UK. p. 54-64.

61. Douglas, J.G. and M.J. McLeod, Pharmacokinetic factors in the modern drug treatment of tuberculosis. Clin Pharmacokinet, 1999. 37(2): p. 127-46.

62. Holdiness, M.R., Clinical pharmacokinetics of the antituberculosis drugs. Clin Pharmacokinet, 1984. 9(6): p. 511-44.

63. Mandell, G.L. and W.A. Petri, Drugs used in the chemotherapy of tuberculosis, Mycobacetrium avium complex disease, and leprosy, in Goodman and Gilman's The Pharmacological Basis of Therapeutics, Hardman, JG and Limbierd, LE; Editors. 1996, McGraw-Hill: New York, NY. p. 111-13.

64. Burman, W.J., K. Gallicano, and C. Peloquin, Comparative pharmacokinetics and pharmacodynamics of the rifamycin antibacterials. Clin Pharmacokinet, 2001. 40(5): p. 327-41.

65. Israili, Z.H., C.M. Rogers, and H. el-Attar, Pharmacokinetics of antituberculosis drugs in patients. J Clin Pharmacol, 1987. 27(1): p. 78-83.

66. Bergogne-Berezin, E., Clinical role of protein binding of quinolones. Clin Pharmacokinet, 2002. 41(10): p. 741-50.

67. Berning, S.E., The role of fluoroquinolones in tuberculosis today. Drugs, 2001. 61(1): p. 9-18.

68. Tomioka, H., H. Saito, and K. Sato, Comparative antimycobacterial activities of the newly synthesized quinolone AM-1155, sparfloxacin, and ofloxacin.

Antimicrob Agents Chemother, 1993. 37(6): p. 1259-63.

69. Balfour, J.A. and H.M. Lamb, Moxifloxacin: a review of its clinical potential in the management of community-acquired respiratory tract infections. Drugs, 2000. 59(1): p. 115-39.

70. U.S.Department of Health and Human Services Food \& Drug Administration, Prescribing information: TEQUIN. 2004, Silver Spring, MD.

71. Diekema, D.I. and R.N. Jones, Oxazolidinones: a review. Drugs, 2000. 59(1): p. 7-16.

72. Guengerich, F.P., Characterization of human microsomal cytochrome P-450 enzymes. Annu Rev Pharmacol Toxicol, 1989. 29: p. 241-64. 
73. Clinical and Laboratory Standards Institute, Approved standard M7-A7: methods for dilution antimicrobial susceptibility test for bBacteria that grow aerobically. 2007: Wayne, PA, USA.

74. Flynn, J.L., et al., Non-human primates: a model for tuberculosis research. Tuberculosis (Edinb), 2003. 83(1-3): p. 116-18.

75. Gupta, U.D. and V.M. Katoch, Animal models of tuberculosis. Tuberculosis (Edinb), 2005. 85(5-6): p. 277-93.

76. Boshoff, H.I. and C.E. Barry, 3rd, Tuberculosis - metabolism and respiration in the absence of growth. Nat Rev Microbiol, 2005. 3(1): p. 70-80.

77. Stover, C.K., et al., A small-molecule nitroimidazopyran drug candidate for the treatment of tuberculosis. Nature, 2000. 405(6789): p. 962-66.

78. Gruppo, V., et al., Rapid microbiologic and pharmacologic evaluation of experimental compounds against Mycobacterium tuberculosis. Antimicrob Agents Chemother, 2006. 50(4): p. 1245-50.

79. Wayne, L.G. and L.G. Hayes, An in vitro model for sequential study of shiftdown of Mycobacterium tuberculosis through two stages of nonreplicating persistence. Infect Immun, 1996. 64(6): p. 2062-69.

80. Woolhiser, L., et al., In vivo adaptation of the Wayne model of latent tuberculosis. Infect Immun, 2007. 75(5): p. 2621-25.

81. Lenaerts, A.J., et al., Location of persisting mycobacteria in a Guinea pig model of tuberculosis revealed by r207910. Antimicrob Agents Chemother, 2007. 51(9): p. 3338-45.

82. Zhang, Y., et al., The catalase-peroxidase gene and isoniazid resistance of Mycobacterium tuberculosis. Nature, 1992. 358(6387): p. 591-93.

83. Peloquin, C.A., Tuberculosis, in Pharmacotherapy: A Pathophysiologic Approach, Dipiro, JT; et al., Editors. 2002, McGraw-Hill: New York, NY. p. 1917-38.

84. Argyrou, A., et al., Proteome-wide profiling of isoniazid targets in Mycobacterium tuberculosis. Biochemistry, 2006. 45(47): p. 13947-53.

85. Winder, F.G. and P.B. Collins, Inhibition by isoniazid of synthesis of mycolic acids in Mycobacterium tuberculosis. J Gen Microbiol, 1970. 63(1): p. 41-48.

86. Nagano, K., et al., Metal complexes of isonicotinoylhydrazine and related compounds. II. Acid dissociation constants and ultraviolet absorption spectra of isonicotinoylhydrazine and related compounds. Chem Pharm Bull (Tokyo), 1963. 11: p. 797-805.

87. Rekker, R.F. and W.T. Nauta, Spectrophotometric investigation of isonicotinoyl hydrazide (Inh). A contribution to the knowledge of the yellow isonicotinoyl hydrazide rho-aminosalicylic acid combination (Inh-Pas). Pharm Weekbl, 1964. 99: p. 1157-65.

88. Becker, C., et al., Biowaiver monographs for immediate release solid oral dosage forms: isoniazid. J Pharm Sci, 2007. 96(3): p. 522-31.

89. Peloquin, C.A., et al., Population pharmacokinetic modeling of isoniazid, rifampin, and pyrazinamide. Antimicrob Agents Chemother, 1997. 41(12): p. 2670-79.

90. Peloquin, C.A., Pharmacology of the antimycobacterial drugs. Med Clin North Am, 1993. 77(6): p. 1253-62. 
91. Weber, W.W. and D.W. Hein, Clinical pharmacokinetics of isoniazid. Clin Pharmacokinet, 1979. 4(6): p. 401-22.

92. Mitchison, D.A., The action of antituberculosis drugs in short-course chemotherapy. Tubercle, 1985. 66(3): p. 219-25.

93. Telenti, A., et al., Detection of rifampicin-resistance mutations in Mycobacterium tuberculosis. Lancet, 1993. 341(8846): p. 647-50.

94. Peloquin, C.A., et al., Pharmacokinetics of rifampin under fasting conditions, with food, and with antacids. Chest, 1999. 115(1): p. 12-18.

95. Seydel, J.K., Physico-chemical studies on rifampicin. Antibiot Chemother, 1970. 16: p. 380-91.

96. Kenny, M.T. and B. Strates, Metabolism and pharmacokinetics of the antibiotic rifampin. Drug Metab Rev, 1981. 12(1): p. 159-218.

97. Bachmann, K.A. and L. Jauregui, Use of single sample clearance estimates of cytochrome P450 substrates to characterize human hepatic CYP status in vivo. Xenobiotica, 1993. 23(3): p. 307-15.

98. Dilger, K., et al., Consequences of rifampicin treatment on propafenone disposition in extensive and poor metabolizers of CYP2D6. Pharmacogenetics, 1999. 9(5): p. 551-59.

99. Gallicano, K.D., et al., Induction of zidovudine glucuronidation and amination pathways by rifampicin in HIV-infected patients. Br J Clin Pharmacol, 1999. 48(2): p. 168-79.

100. Greiner, B., et al., The role of intestinal P-glycoprotein in the interaction of digoxin and rifampin. J Clin Invest, 1999. 104(2): p. 147-53.

101. Caraco, Y., J. Sheller, and A.J. Wood, Pharmacogenetic determinants of codeine induction by rifampin: the impact on codeine's respiratory, psychomotor and miotic effects. J Pharmacol Exp Ther, 1997. 281(1): p. 330-36.

102. Tarshis, M.S. and W.A. Weed, Jr., Lack of significant in vitro sensitivity of Mycobacterium tuberculosis to pyrazinamide on three different solid media. Am Rev Tuberc, 1953. 67(3): p. 391-95.

103. Wade, M.M. and Y. Zhang, Anaerobic incubation conditions enhance pyrazinamide activity against Mycobacterium tuberculosis. J Med Microbiol, 2004. 53(Pt 8): p. 769-73.

104. Zhang, Y., S. Permar, and Z. Sun, Conditions that may affect the results of susceptibility testing of Mycobacterium tuberculosis to pyrazinamide. J Med Microbiol, 2002. 51(1): p. 42-49.

105. Mandell, G.L. and W.A. Petri, Antimicrobial agents: Drugs used in chemotherapy of tuberculosis, Mycobacterium avium complex disease, and leprosy, in Goodman and Gilman's The Pharmacological Basis of Therapeutics, Hardman, JG; et al., Editors. 1996, McGraw-Hill: New York, NY. p. 1161-62.

106. Scorpio, A. and Y. Zhang, Mutations in pncA, a gene encoding pyrazinamidase/nicotinamidase, cause resistance to the antituberculous drug pyrazinamide in tubercle bacillus. Nat Med, 1996. 2(6): p. 662-67.

107. Scorpio, A., et al., Characterization of pncA mutations in pyrazinamide-resistant Mycobacterium tuberculosis. Antimicrob Agents Chemother, 1997. 41(3): p. 54043. 
108. Felder, E. and D. Pitre, Pyrazinamide, in Analytical Profiles of Drug Substances. 1983, Academic Press: London, UK. p. 433-63.

109. Takayama, K. and J.O. Kilburn, Inhibition of synthesis of arabinogalactan by ethambutol in Mycobacterium smegmatis. Antimicrob Agents Chemother, 1989. 33(9): p. 1493-99.

110. Telenti, A., et al., The emb operon, a gene cluster of Mycobacterium tuberculosis involved in resistance to ethambutol. Nat Med, 1997. 3(5): p. 567-70.

111. Kanther, R., Myambutol: chemistry, pharmacology, and toxicology. Antibiot Chemother, 1970. 16: p. 203-14.

112. Peets, E.A., et al., The absorption, excretion, and metabolic fate of ethambutol in man. Am Rev Respir Dis, 1965. 91: p. 51-58.

113. Hughes, N.C., et al., Identification and characterization of variant alleles of human acetyltransferase NAT1 with defective function using p-aminosalicylate as an in-vivo and in-vitro probe. Pharmacogenetics, 1998. 8(1): p. 55-66.

114. DeBarber, A.E., et al., Ethionamide activation and sensitivity in multidrugresistant Mycobacterium tuberculosis. Proc Natl Acad Sci U S A, 2000. 97(17): p. 9677-82.

115. Baulard, A.R., et al., Activation of the pro-drug ethionamide is regulated in mycobacteria. J Biol Chem, 2000. 275(36): p. 28326-31.

116. Morlock, G.P., et al., ethA, inhA, and katG loci of ethionamide-resistant clinical Mycobacterium tuberculosis isolates. Antimicrob Agents Chemother, 2003. 47(12): p. 3799-805.

117. Jacobs, M.R., Activity of quinolones against mycobacteria. Drugs, 1995. 49 Suppl 2: p. 67-75.

118. David, H.L., K. Takayama, and D.S. Goldman, Susceptibility of mycobacterial Dalanyl-D-alanine synthetase to D-cycloserine. Am Rev Respir Dis, 1969. 100(4): p. 579-81.

119. Strych, U., et al., Characterization of the alanine racemases from two mycobacteria. FEMS Microbiol Lett, 2001. 196(2): p. 93-98.

120. Strausbaugh, L.J., C.D. Mandaleris, and M.A. Sande, Comparison offour aminoglycoside antibiotics in the therapy of experimental E. coli meningitis. J Lab Clin Med, 1977. 89(4): p. 692-701.

121. Elliott, A.M. and S.D. Foster, Thiacetazone: time to call a halt? Considerations on the use of thiacetazone in African populations with a high prevalence of human immunodeficiency virus infection. Tuber Lung Dis, 1996. 77(1): p. 27-29.

122. Mor, N., et al., Comparison of activities of rifapentine and rifampin against Mycobacterium tuberculosis residing in human macrophages. Antimicrob Agents Chemother, 1995. 39(9): p. 2073-77.

123. Reith, K., et al., Disposition and metabolism of 14C-rifapentine in healthy volunteers. Drug Metab Dispos, 1998. 26(8): p. 732-38.

124. Jarvis, B. and H.M. Lamb, Rifapentine. Drugs, 1998. 56(4): p. 607-16; discussion 617.

125. Gillespie, S.H. and O. Billington, Activity of moxifloxacin against mycobacteria. J Antimicrob Chemother, 1999. 44(3): p. 393-95. 
126. Ji, B., et al., In vitro and in vivo activities of moxifloxacin and clinafloxacin against Mycobacterium tuberculosis. Antimicrob Agents Chemother, 1998. 42(8): p. 2066-69.

127. Miyazaki, E., et al., Moxifloxacin (BAY12-8039), a new 8-methoxyquinolone, is active in a mouse model of tuberculosis. Antimicrob Agents Chemother, 1999. 43(1): p. 85-89.

128. Pletz, M.W., et al., Early bactericidal activity of moxifloxacin in treatment of pulmonary tuberculosis: a prospective, randomized study. Antimicrob Agents Chemother, 2004. 48(3): p. 780-82.

129. U.S. Department of Health and Human Services Food \& Drug Administration, Prescribing information: AVELOX. 2004, Silver Spring, MD.

130. Tomioka, H., Current status of some antituberculosis drugs and the development of new antituberculous agents with special reference to their in vitro and in vivo antimicrobial activities. Curr Pharm Des, 2006. 12(31): p. 4047-70.

131. Paramasivan, C.N., et al., Bactericidal action of gatifloxacin, rifampin, and isoniazid on logarithmic- and stationary-phase cultures of Mycobacterium tuberculosis. Antimicrob Agents Chemother, 2005. 49(2): p. 627-31.

132. Zhao, B.Y., et al., Fluoroquinolone action against clinical isolates of Mycobacterium tuberculosis: effects of a C-8 methoxyl group on survival in liquid media and in human macrophages. Antimicrob Agents Chemother, 1999. 43(3): p. 661-66.

133. Alcala, L., et al., In vitro activities of linezolid against clinical isolates of Mycobacterium tuberculosis that are susceptible or resistant to first-line antituberculous drugs. Antimicrob Agents Chemother, 2003. 47(1): p. 416-17.

134. von der Lippe, B., P. Sandven, and O. Brubakk, Efficacy and safety of linezolid in multidrug resistant tuberculosis (MDR-TB)--a report of ten cases. J Infect, 2006. 52(2): p. 92-96.

135. Fortun, J., et al., Linezolid for the treatment of multidrug-resistant tuberculosis. J Antimicrob Chemother, 2005. 56(1): p. 180-85.

136. Park, I.N., et al., Efficacy and tolerability of daily-half dose linezolid in patients with intractable multidrug-resistant tuberculosis. J Antimicrob Chemother, 2006. 58(3): p. 701-14.

137. U.S. Department of Health and Human Services Food \& Drug Administration, Prescribing information: ZYVOX. 2005, Silver Spring, MD.

138. Wayne, L.G. and H.A. Sramek, Metronidazole is bactericidal to dormant cells of Mycobacterium tuberculosis. Antimicrob Agents Chemother, 1994. 38(9): p. 2054-58.

139. Brooks, J.V., S.K. Furney, and I.M. Orme, Metronidazole therapy in mice infected with tuberculosis. Antimicrob Agents Chemother, 1999. 43(5): p. 128588.

140. Dhillon, J., et al., Metronidazole has no antibacterial effect in Cornell model murine tuberculosis. Int J Tuberc Lung Dis, 1998. 2(9): p. 736-42.

141. Walsh, G.P., et al., The Philippine cynomolgus monkey (Macaca fasicularis) provides a new nonhuman primate model of tuberculosis that resembles human disease. Nat Med, 1996. 2(4): p. 430-36. 
142. U.S. National Institutes of Health ClinicalTrials.Gov. Metronidazole for pulmonary tuberculosis (South Korea). 2007 [cited Dec 15, 2007]; Available from: http://clinicaltrials.gov/ct/show/NCT00425113?order=1.

143. U.S. Department of Health and Human Services Food \& Drug Administration, Prescribing information: FLAGYL. 2003, Silver Spring, MD.

144. Nuermberger, E., et al., Combination chemotherapy with the nitroimidazopyran PA-824 and first-line drugs in a murine model of tuberculosis. Antimicrob Agents Chemother, 2006. 50(8): p. 2621-25.

145. Ahmad, K., New tuberculosis drug enters human trials. Lancet Infect Dis, 2005. 5(8): p. 475.

146. Lillibridge, J.H., et al., PA-824, a nitroimidazopyran antitubercular agent: favorable pharmacokinetics parameters in mice after delivery in a novel oral formulation. 1996: Abstr Intersci Conf Antimicrob Agents Chemother. 137 (abstract no. F216).

147. Sasaki, H., et al., Synthesis and antituberculosis activity of a novel series of optically active 6-nitro-2,3-dihydroimidazo[2,1-b]oxazoles. J Med Chem, 2006. 49(26): p. 7854-60.

148. Matsumoto, M., et al., OPC-67683, a nitro-dihydro-imidazooxazole derivative with promising action against tuberculosis in vitro and in mice. PLoS Med, 2006. 3(11): p. e466.

149. Hittel, N., Open forum II on key issues in TB drug development. 2006 [cited 15 March, 2007]; Available from: http://www.kaisernetwork.org.

150. Koul, A., et al., Diarylquinolines target subunit c of mycobacterial ATP synthase. Nat Chem Biol, 2007. 3(6): p. 323-24.

151. Cole, S.T. and P.M. Alzari, Microbiology. TB--a new target, a new drug. Science, 2005. 307(5707): p. 214-15.

152. Budha, N.R., R.E. Lee, and B. Meibohm, Biopharmaceutics, pharmacokinetics and pharmacodynamics of antituberculosis drugs. Curr Med Chem, 2008. 15(8): p. 809-25.

153. Ibrahim, M., et al., Synergistic activity of R207910 combined with pyrazinamide against murine tuberculosis. Antimicrob Agents Chemother, 2007. 51(3): p. 1011-15.

154. Mc Neely, D.F. and M. Cavaleri, TMC207 Oral Presentation. 2005, San Diego, CA: TBTC 17th Semi-Annual Group Meeting.

155. U.S. National Institutes of Health ClinicalTrials.Gov. TMC207-TiDP13-C208: Anti-bacterial activity, safety, and tolerability of TMC207 in patients with multidrug resistant Mycobacterium tuberculosis (MDR-TB). 2007 [cited 23 May, 2007]; Available from: http://clinicaltrials.gov/ct/show/NCT00449644?order=1.

156. Guengerich, F.P., Cytochrome P-450 3A4: regulation and role in drug metabolism. Annu Rev Pharmacol Toxicol, 1999. 39: p. 1-17.

157. Ginsberg, A.M. and M. Spigelman, Challenges in tuberculosis drug research and development. Nat Med, 2007. 13(3): p. 290-94.

158. Lee, R.E., et al., Combinatorial lead optimization of [1,2]-diamines based on ethambutol as potential antituberculosis preclinical candidates. J Comb Chem, 2003. 5(2): p. 172-87. 
159. Jia, L., et al., Pharmacodynamics and pharmacokinetics of SQ109, a new diamine-based antitubercular drug. Br J Pharmacol, 2005. 144(1): p. 80-87.

160. Nikonenko, B.V., et al., Drug therapy of experimental tuberculosis (TB): improved outcome by combining SQ109, a new diamine antibiotic, with existing TB drugs. Antimicrob Agents Chemother, 2007. 51(4): p. 1563-35.

161. Jia, L., et al., Interspecies pharmacokinetics and in vitro metabolism of SQ109. $\mathrm{Br}$ J Pharmacol, 2006. 147(5): p. 476-85.

162. NIAID-Division of AIDS, HIV/OI Therapeutics Database. 2007, National Institute of Allergy and Infectious Diseases (NIAID).

163. Cruciani, G., M. Pastor, and W. Guba, VolSurf: a new tool for the pharmacokinetic optimization of lead compounds. Eur J Pharm Sci, 2000. 11 Suppl 2: p. S29-39.

164. Lillibridge, J.H., et al., PA-824, a nitroimidazopyran antitubercular agent: favorable pharmacokinetic parameters in mice after delivery in a novel oral formulation, in 36th Annual Interscience conference on Antimicrobial Agents and Chemotherapy (ICAAC). 1996: New Orleans, Louisiana.

165. Craig, W.A., Pharmacokinetic/pharmacodynamic parameters: rationale for antibacterial dosing of mice and men. Clin Infect Dis, 1998. 26(1): p. 1-10; quiz 11-12.

166. Wright, D.H., et al., Application of fluoroquinolone pharmacodynamics. J Antimicrob Chemother, 2000. 46(5): p. 669-83.

167. Jayaram, R., et al., Isoniazid pharmacokinetics-pharmacodynamics in an aerosol infection model of tuberculosis. Antimicrob Agents Chemother, 2004. 48(8): p. 2951-57.

168. Jayaram, R., et al., Pharmacokinetics-pharmacodynamics of rifampin in an aerosol infection model of tuberculosis. Antimicrob Agents Chemother, 2003. 47(7): p. 2118-24.

169. Shandil, R.K., et al., Moxifloxacin, ofloxacin, sparfloxacin, and ciprofloxacin against Mycobacterium tuberculosis: evaluation of in vitro and pharmacodynamic indices that best predict in vivo efficacy. Antimicrob Agents Chemother, 2007. 51(2): p. 576-82.

170. Nuermberger, E. and J. Grosset, Pharmacokinetic and pharmacodynamic issues in the treatment of mycobacterial infections. Eur J Clin Microbiol Infect Dis, 2004. 23(4): p. 243-55.

171. World Health Organization, WHO Drug Information, Publications, Editor. 2006, W.H.O. p. 239-45.

172. Nightingale, C.H., R. Quintiliani, and D.P. Nicolau, Intelligent dosing of antimicrobials, in Current Clinical Topics in Infectious Diseases, Remington, JS and Swartz, MN; Editors. 1994, Blackwell Scientific: Cambridge, MA. p. 252-65.

173. Schentag, J.J., Pharmacokinetic and pharmacodynamic predictors of antimicrobial efficacy: moxifloxacin and Streptococcus pneumoniae. J Chemother, 2002. 14 Suppl 2: p. 13-21.

174. Delacher, S., et al., A combined in vivo pharmacokinetic-in vitro pharmacodynamic approach to simulate target site pharmacodynamics of antibiotics in humans. J Antimicrob Chemother, 2000. 46(5): p. 733-39. 
175. Bonate, P.L., Clinical trial simulation in drug development. Pharm Res, 2000. 17(3): p. 252-56.

176. Meibohm, B. and H. Derendorf, Pharmacokinetic/pharmacodynamic studies in drug product development. J Pharm Sci, 2002. 91(1): p. 18-31.

177. CDER/FDA, Exposure Response Relationships: Guidance for Industry, US Department of Health and Human Services, Food and Drug Administration, Center for Drug Evaluation and Research, Rockville, Editor. 2003.

178. Lesko, L.J., et al., Optimizing the science of drug development: opportunities for better candidate selection and accelerated evaluation in humans. J Clin Pharmacol, 2000. 40(8): p. 803-14.

179. Peck, C.C., et al., Opportunities for integration of pharmacokinetics, pharmacodynamics, and toxicokinetics in rational drug development. J Clin Pharmacol, 1994. 34(2): p. 111-19.

180. Svensson, U.S., et al., Population pharmacokinetic and pharmacodynamic modelling of artemisinin and mefloquine enantiomers in patients with falciparum malaria. Eur J Clin Pharmacol, 2002. 58(5): p. 339-51.

181. Schentag, J.J., A.K. Meagher, and A. Forrest, Fluoroquinolone AUIC break points and the link to bacterial killing rates. Part 1: In vitro and animal models. Ann Pharmacother, 2003. 37(9): p. 1287-98.

182. Schentag, J.J., A.K. Meagher, and A. Forrest, Fluoroquinolone AUIC break points and the link to bacterial killing rates. Part 2: human trials. Ann Pharmacother, 2003. 37(10): p. 1478-88.

183. Schuck, E.L., et al., Pharmacokinetic/pharmacodynamic (PK/PD) evaluation of a once-daily treatment using ciprofloxacin in an extended-release dosage form. Infection, 2005. 33 Suppl 2: p. 22-28.

184. Regoes, R.R., et al., Pharmacodynamic functions: a multiparameter approach to the design of antibiotic treatment regimens. Antimicrob Agents Chemother, 2004. 48(10): p. 3670-76.

185. Li, Y., et al., A pharmacokinetic/pharmacodynamic mathematical model accurately describes the activity of voriconazole against Candida spp. in vitro. Int J Antimicrob Agents, 2008. 31(4): p. 369-74.

186. Boak, L.M., et al., Pharmacokinetic/pharmacodynamic factors influencing emergence of resistance to linezolid in an in vitro model. Antimicrob Agents Chemother, 2007. 51(4): p. 1287-92.

187. Blumberg, H.M., et al., ATS/CDC/IDSA: Treatment of tuberculosis. Am J Respir Crit Care Med, 2003. 167(4): p. 603-62.

188. Burman, W.J. and B.E. Jones, Treatment of HIV-related tuberculosis in the era of effective antiretroviral therapy. Am J Respir Crit Care Med, 2001. 164(1): p. 712.

189. Tangallapally, R.P., et al., Synthesis and evaluation of nitrofuranylamides as novel antituberculosis agents. J Med Chem, 2004. 47(21): p. 5276-83.

190. Tangallapally, R.P., et al., Synthesis and evaluation of cyclic secondary amine substituted phenyl and benzyl nitrofuranyl amides as novel antituberculosis agents. J Med Chem, 2005. 48(26): p. 8261-69.

191. Tangallapally, R.P., et al., Synthesis of new and potent analogues of antituberculosis agent 5-nitro-furan-2-carboxylic acid 4-(4-benzyl-piperazin-1-yl)- 
benzylamide with improved bioavailability. Bioorg Med Chem Lett, 2006. 16(10): p. 2584-89.

192. Tangallapally, R.P., et al., Discovery of novel isoxazolines as anti-tuberculosis agents. Bioorg Med Chem Lett, 2007. 17(23): p. 6638-42

193. Laughon, B.E., New tuberculosis drugs in development. Curr Top Med Chem, 2007. 7(5): p. 463-73.

194. Eddershaw, P.J., A.P. Beresford, and M.K. Bayliss, ADME/PK as part of a rational approach to drug discovery. Drug Discov Today, 2000. 5(9): p. 409-14.

195. Tangallapally, R.P., et al., Nitrofurans as novel anti-tuberculosis agents: identification, development and evaluation. Curr Top Med Chem, 2007. 7(5): p. 509-26.

196. Glomme, A., J. Marz, and J.B. Dressman, Comparison of a miniaturized shakeflask solubility method with automated potentiometric acid/base titrations and calculated solubilities. J Pharm Sci, 2005. 94(1): p. 1-16.

197. Thompson, T.N., Optimization of metabolic stability as a goal of modern drug design. Med Res Rev, 2001. 21(5): p. 412-49.

198. White, R.E., High-throughput screening in drug metabolism and pharmacokinetic support of drug discovery. Annu Rev Pharmacol Toxicol, 2000. 40: p. 133-57.

199. Davies, B. and T. Morris, Physiological parameters in laboratory animals and humans. Pharm Res, 1993. 10(7): p. 1093-95.

200. Strickley, R.G., Solubilizing excipients in oral and injectable formulations. Pharm Res, 2004. 21(2): p. 201-30.

201. Mueller, M., A. de la Pena, and H. Derendorf, Issues in pharmacokinetics and pharmacodynamics of anti-infective agents: kill curves versus MIC. Antimicrob Agents Chemother, 2004. 48(2): p. 369-77.

202. The U.S. Department of Health and Human Services Food and Drug Administration, Developing antimicribial drugs-general considerations for clinical trials. 1998, Rockville, MD.

203. The European Agency for the Evaluation of Medicinal Products (EMEA) Committee for Proprietary Medicinal Products (CPMP), Points to consider on pharmacokinetics and pharmacodynamics in the development of antibacterial medicinal products. 2000, London, UK.

204. Lowdin, E., et al., Pharmacodynamic effects of sub-MICs of benzylpenicillin against Streptococcus pyogenes in a newly developed in vitro kinetic model. Antimicrob Agents Chemother, 1996. 40(11): p. 2478-82.

205. Dalla Costa, T., et al., Pharmacokinetic-pharmacodynamic modelling of the in vitro antiinfective effect of piperacillin-tazobactam combinations. Int J Clin Pharmacol Ther, 1997. 35(10): p. 426-33.

206. Schuck, E.L. and H. Derendorf, Pharmacokinetic/pharmacodynamic evaluation of anti-infective agents. Expert Rev Anti Infect Ther, 2005. 3(3): p. 361-73.

207. Muller, M., A. dela Pena, and H. Derendorf, Issues in pharmacokinetics and pharmacodynamics of anti-infective agents: distribution in tissue. Antimicrob Agents Chemother, 2004. 48(5): p. 1441-53.

208. Lister, P.D., The role of pharmacodynamic research in the assessment and development of new antibacterial drugs. Biochem Pharmacol, 2006. 71(7): $\mathrm{p}$. 1057-65. 
209. Grasso, S., et al., New in vitro model to study the effect of antibiotic concentration and rate of elimination on antibacterial activity. Antimicrob Agents Chemother, 1978. 13(4): p. 570-76.

210. Maggiolo, F., et al., Bactericidal activity of two different dosage regimens of imipenem in an in-vitro dynamic model. J Antimicrob Chemother, 1993. 32(2): p. 295-300.

211. Murakawa, T., et al., New in vitro kinetic model for evaluating bactericidal efficacy of antibiotics. Antimicrob Agents Chemother, 1980. 18(3): p. 377-81.

212. Gumbo, T., et al., Isoniazid bactericidal activity and resistance emergence: integrating pharmacodynamics and pharmacogenomics to predict efficacy in different ethnic populations. Antimicrob Agents Chemother, 2007. 51(7): p. 232936.

213. Gumbo, T., et al., Selection of a moxifloxacin dose that suppresses drug resistance in Mycobacterium tuberculosis, by use of an in vitro pharmacodynamic infection model and mathematical modeling. J Infect Dis, 2004. 190(9): p. 164251.

214. Gumbo, T., et al., Concentration-dependent Mycobacterium tuberculosis killing and prevention of resistance by rifampin. Antimicrob Agents Chemother, 2007. 51(11): p. 3781-88.

215. Gumbo, T., et al., Isoniazid's bactericidal activity ceases because of the emergence of resistance, not depletion of Mycobacterium tuberculosis in the log phase of growth. J Infect Dis, 2007. 195(2): p. 194-201.

216. Middlebrook, G., Isoniazid-resistance and catalase activity of tubercle bacilli; a preliminary report. Am Rev Tuberc, 1954. 69(3): p. 471-72.

217. Viveiros, M., et al., Isoniazid-induced transient high-level resistance in Mycobacterium tuberculosis. Antimicrob Agents Chemother, 2002. 46(9): p. 2804-10.

218. Colangeli, R., et al., The Mycobacterium tuberculosis iniA gene is essential for activity of an efflux pump that confers drug tolerance to both isoniazid and ethambutol. Mol Microbiol, 2005. 55(6): p. 1829-40.

219. Wayne, L.G., Mycobacterial speciation, in The Mycobacteria: A Sourcebook, Kubica, GP and Wayne, LG; Editors. 1984, Marcel Dekker: New York, NY. p. 25-66.

220. Yano, Y., et al., Application of logistic growth model to pharmacodynamic analysis of in vitro bactericidal kinetics. J Pharm Sci, 1998. 87(10): p. 1177-83.

221. Nielsen, E.I., et al., Semimechanistic pharmacokinetic/pharmacodynamic model for assessment of activity of antibacterial agents from time-kill curve experiments. Antimicrob Agents Chemother, 2007. 51(1): p. 128-36.

222. Carret, G., J.P. Flandrois, and J.R. Lobry, Biphasic kinetics of bacterial killing by quinolones. J Antimicrob Chemother, 1991. 27(3): p. 319-27.

223. Meagher, A.K., et al., Novel pharmacokinetic-pharmacodynamic model for prediction of outcomes with an extended-release formulation of ciprofloxacin. Antimicrob Agents Chemother, 2004. 48(6): p. 2061-68.

224. Mouton, J.W., A.A. Vinks, and N.C. Punt, Pharmacokinetic-pharmacodynamic modeling of activity of ceftazidime during continuous and intermittent infusion. Antimicrob Agents Chemother, 1997. 41(4): p. 733-38. 
225. Tam, V.H., et al., Mathematical modelling response of Pseudomonas aeruginosa to meropenem. J Antimicrob Chemother, 2007. 60(6): p. 1302-09.

226. Liu, P., et al., Pharmacokinetic-pharmacodynamic modelling of antibacterial activity of cefpodoxime and cefixime in in vitro kinetic models. Int J Antimicrob Agents, 2005. 25(2): p. 120-29.

227. Tam, V.H., A.N. Schilling, and M. Nikolaou, Modelling time-kill studies to discern the pharmacodynamics of meropenem. J Antimicrob Chemother, 2005. 55(5): p. 699-706.

228. Zhi, J., C.H. Nightingale, and R. Quintiliani, A pharmacodynamic model for the activity of antibiotics against microorganisms under nonsaturable conditions. $\mathrm{J}$ Pharm Sci, 1986. 75(11): p. 1063-67.

229. Jindani, A., et al., The early bactericidal activity of drugs in patients with pulmonary tuberculosis. Am Rev Respir Dis, 1980. 121(6): p. 939-49.

230. Jindani, A., C.J. Dore, and D.A. Mitchison, Bactericidal and sterilizing activities of antituberculosis drugs during the first 14 days. Am J Respir Crit Care Med, 2003. 167(10): p. 1348-54.

231. McClatchy, J.K., Mechanism of action of isoniazid on Mycobacterium bovis strain BCG. Infect Immun, 1971. 3(4): p. 530-34.

232. Wallis, R.S., et al., Drug tolerance in Mycobacterium tuberculosis. Antimicrob Agents Chemother, 1999. 43(11): p. 2600-06.

233. Mouton, J.W. and A.A. Vinks, Pharmacokinetic/pharmacodynamic modelling of antibacterials in vitro and in vivo using bacterial growth and kill kinetics: the minimum inhibitory concentration versus stationary concentration. Clin Pharmacokinet, 2005. 44(2): p. 201-10.

234. Hurdle, J.G., et al., A microbiological assessment of novel nitrofuranylamides as anti-tuberculosis agents. J Antimicrob Chemother, 2008. 62(5): p. 1037-45.

235. Budha, N.R., et al., Pharmacokinetically-guided lead optimization of nitrofuranylamide anti-tuberculosis agents. AAPS J, 2008. 10(1): p. 157-65.

236. Burman, W.J., Issues in the management of HIV-related tuberculosis. Clin Chest Med, 2005. 26(2): p. 283-94, vi-vii.

237. Eagle, H. and A.D. Musselman, The rate of bactericidal action of penicillin in vitro as a function of its concentration, and its paradoxically reduced activity at high concentrations against certain organisms. J Exp Med, 1948. 88(1): p. 99131.

238. West, G.B., J.H. Brown, and B.J. Enquist, A general model for the origin of allometric scaling laws in biology. Science, 1997. 276(5309): p. 122-26.

239. Boxenbaum, H., Interspecies scaling, allometry, physiological time, and the ground plan of pharmacokinetics. J Pharmacokinet Biopharm, 1982. 10(2): p. 201-27. 


\section{VITA}

Nageshwar R. Budha was born in Warangal, Andhra Pradesh, India, in 1976. He joined the Kakatiya University in 1997 and received a Bachelor in Pharmacy degree in 2001. He joined in the same university for Master's program in pharmaceutics in 2002 and graduated in 2004. In the Fall of 2004, he joined the graduate program in the Department of Pharmaceutical Sciences, the University of Tennessee Health Science Center, Memphis, from where he will receive his Ph.D. under the supervision of Prof. Bernd Meibohm. 